PM

2496

Z77U55

1873

SOA

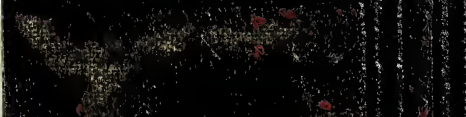


$\sqrt{4})^{3}$ 
hang on plen

frrm 



\title{
A SELECTION
}

\author{
FROM THE
}

\section{BOOK OF COMMON PRAYER,}

ACCORDING TO THE USE OF THE

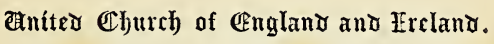

TRA NSLATED INTO

\section{TUKUDH,}

BY THE REV. R. M'DONALD,

MISSTONARY OF THE CHURCH MISSIONARY SOCIETY.

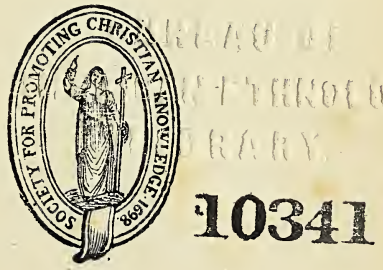

\section{LONDON :}

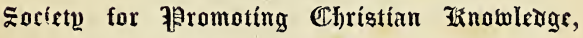

77, GREAT QUEEN STRHET, LINCOLN'S-INN-FIELDS ;

4, royal EXchange; aNd 48, piccadilly.

1873. 


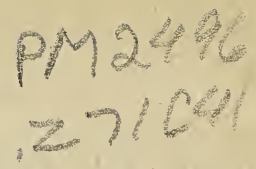

LONDON :

PRINTED BY GILBERT AND RIVINGTON, ST. JOHN'S SQUARE, AND 28, WHITEFRIARS STREET.

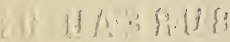

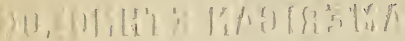


THF

\section{ORDER FOR MORNING PRAYER}

DAILY THROUGHOUT THE YEAR.

\section{นาน \\ VUNTAT TRIGIKHYI}

NUKWUTUTHUD TUTTHUG KENJIT.

ITCHITAT tinjih trandyọh ei troogwanIJ dyoh tetiyin akhekonyoo, akọ ei rsittuttsut ako tshoogoochöe tsut titiyin tuh nkyo tassi. Ezek. xviii. 27.

Suh troogwandyoh kwulhnduk, akọ suh troogwandyoh suh ndah sheg kooei. $P s$. li. 3.

Suh troogwandyoh kwu ttsut nih nyun ninyai, ako tutthug suh troogwandyoh oounkoochịli. Ps. li. 9.

Kineinjitowhohchi : kwugguh yoo zyeh kug kwu ttsut kookukwadhud tsuh nyakwuth. St. Matt. iii. 2.

Nutihseh akọ ttia vuh tsuh tihsyah, ako akotuvutakhnja: Ttia zyeh zit kwu tsut akọ nyih ndah troogwandyoh kwudhelhtseị : akọ tthui tte elyet giihthlị sih tinji suh tạnja. St. Luke xv. 18, 19.

Elyet troogwandyoh kundhitii zyunchitinyoo chi, zyunnettitinitaë, akọ elyet thliëteg1 
witli nuwhoh zit kwilị. Nuwhoh troogwandyoh keh zyunkwanduk chi, thlih ha ako tshitchoë nili nuwhoh troogwandyoh nuwheh ooun kootelya, ako tutthug troogwandyoh kwu ttsut nuwhun uttata. 1 John i. 8,9.

T WINTLUTH nuwhet tunyinutudhun 1. kwuchanut,Etunetle choh kwi zit nikhun kwitekit akọtunnuwhahnyoo nuwhoh troogwandyoh kwilyei zjunkwutanduk : ako elyet tunnyinudhaä Vittukoochanchyo thlạchi hs nichinttai zyeh kug nuwhoh Ttyi vuh ndah: kọ kwittri chettukotithinyoo, thlitseyinjikwitunzhit, ako kookukwathut ha; ei kwa tsut nuwhoh troogwandyọ ekooteta, vih chetrsikoonchyo akọ vih rsinichi ha kirre. Ako Vittukoochanchyo ndah chettukotithinyoo ha - nuwhoh troogwandyoh sheg zyunkwanduk chi; kwuttuzug kwiyendo tsut akotunitiyin chi, etchittaị chunzyunnithlichazi huị vuh ntlunchitaë tutthug vih chehrsikoonchyo kenjit, vih chile akotuntanja, ako zyunotutakhut tutthug ei ekoochichyo tinjikwitizhit kwizyin akọ kwunkyo enjit. Azyuk kwuggut akotunnuwhohchikhnyoo nuwhot tatthug tzet zyunohthlị sih kit teigokhyah kwittri rsittuttsut ako kwitezyoo chettukotithinyoo ha zyeh kug tetletchiti chetrsikoonchyo kwu tsut, akọtunchitinyoo:

- Kehkwanduk thlachya tutthug akọtungititenja Gidhyi ha, tutthug nuntshikiouttrule.

THLẠCHI ha nichinttai tinichyo ako kwit1 tyi kwintluth nyetrsikoonchyo: Nyih 
netinde kwuttsun thlettinidhidichil, ako tettinidhidichil tivi hazyuntrookwetunduk kit. Nittso nuwhot ttri zit nunyinjidhidichik akọ tunyinjidhidichik treshit kwikit tedhidichil. Nih kinjik rsotitinyoo tsut koottsite zyunkwudhatseị. Elyet akọtungwaya ei akọtungwayin chi : akotungwayin ei elyet akọtungwaya chi : akọ nuwhoh zit kwịrzị kookwundai kwilị. Kọ nun Kukwạdhud nersigoochachyo tunnuwhoh chịnyoo huịnjiidichi troogwandyoh akọtungwayị. O Vittukoochanchyo rsyunkoohchanchyo einut kuttuh troogwandyoh nyih ndah keh zyunkookwanduk. Chun kwettunyinịdhun nih tsuh kinugutta; nyih khenitteikwichittäei kit tinjih tsut nuwhẹh Kukwadhud Jesus Christ kirre. Akọ Kwittyi kwintluth nyetrsikoonchyo, vih kirre akotunnuwhohchinyoo; choog kwu ttsut shọ elyet zyunkwutitundai nih kinjik kit, tshoogoochöe tsut, akọ rsittuttsut, nindelh tsut nyooh rzi rsotitinyoo. Amen.

9 Troogwandyoh ekoodichya, Gikhyi rzi nudhut akotititenja ; tinjih tthuik tte nuntshikwuttrule.

THLANCHI ha nichinttai Vittukoochan1 chyo, Kwittyi nuwheh Kukwadhud Jesus Christ, ẹi yoo elyet akọtinidhun ihthle trandyoh nutedha, Ko kwiyendo tsut yih troogwandyoh akhekotitenja ako kwutendai : ako kwuttai ako kettugwichiltshei tih gịhyinut kwuntlutetäei, akọtungititenja akọ kunkwutanduk tih tsyahnut kooh troogwandyoh akhekotitinyoo: Ei yoo tutthug einut thlih 
ha thlitsetunyinjigichi kooh troogwandyoh oounkootili. Azyuk kwuggut vuh tsuh zyungittinichi thlih ha thlitseyinjikwitizhit nuwhoh ntlutetuä, ako vuh Nkyo Rsotitinyoo, ei ekoochichyo choog tunitiyin sso vuh tsun yih tatsya, ako choog kwu ttsut nuwhoh kwundai rsikotitinyoo akọ kwịrzị kwitelya: akọ tukoonchyo huịko tsut sheggu vih ssokoọlị nutituta; Jesus Christ nuwheh Kukwadhud kirre. Amen.

9ा Tzet ako tutthug trigikhyi kwittsi, tinji akotungititenja, Amen.

बा Ako tle Gikhgi nitshikwuttuttä̈, ako Kukwadhud kwigikhyi ahotititenja ; tinji tthuih nuntshikwuttrule, gih kit teikigikhyi.

TUWHOT Ttyi zyeh zit nih kwilnjik. 1 Nyooh rzi chootsi. Nih kookukwadhud nuvizi. Tinidhun nunh kuggu tugoochyo nittso zyeh zit troogwatsi. Chih ttrin zit nuwhun enyantsit ttrin ndo thloogootunazya nuwhoh ssih. Nuwhot troogwandyoh nuwheh unkoochịli nittso unkoochitili einut troogwandyoh nuwheh tungitiyin. Koọut-kukwadhud kwu tsut nuwhoh tunọe rshọ: kọ troogwandyoh kwu ttsut nuwhoh yunninji. Kwugguh yoo nih ttsun nilị kookukwadhud, ttai, akọ nindelh sheggu kenjit akọ sheggu kenjit. Amen.

\section{Jubilate Deo. Psalm or Chilig C.}

MUTTHUG Kthoikit Kukwadhud ha sso1 zyuhohthlị : sso ha Kukwadhud zyun4 
konduk, akọ vuh ndah nunohchya chilig ha.

Kwikyinjohchi Kukwadhud Vittukoochanchyo nilị: ei yoo nuwheltseị, akọ nuwhunthun ukkwa; vih tsyahnut ako vuh ttsun chutrua kwitekit kwi tivi zyunitili.

Vih kittenivya kwizit sso ha nitetohchya, akọ vih zze kwi zit chilig ha: hui vet zyunyinoohthun, akọ vooh rzi keh njit krwịrzị zyungohkhyah.

Kwugguh yoo Kukwadhud veh rsikoonchyo nili, vih chetrsikoonchyo sheggu koọlị : akọ vih thliëtegwitli nizyoọnedichi kwu ttsut nizyoọ nedichi tsut.

Kwittyi akọ Kwitinji : akọ Chunkyo Rsotitinyoo ha chịnttsi kolị ;

Nittșo koogwinyathud Kwịlịh, chooggoo koọli, akọ sheggu kenjit kwittelya; nunh kwilikwutetha kwu tsut. Amen.

I Akọtle Chilehnut koole kwikyinjigwizhit akọtititetinja Gikhyitinjihnut ha nugichilai kirre.

K WIKYINJIHSHIT Vittukoochanchyo I koọ Kwittyi Thlanchi ha nichinttai, yzeh nunh ha eltsei :

Akọ ei rzi vih Tinji Jesus Christ nuwheh Kukwadhud, Ei Chunkyo Rsotitinyoo kirre tinjih uittsei, Nichid Mary ttsut vih kwịlịh, Pontius Pilate teggug huịjithudhut, Tuchun kwinititaë kug gulttsuk, ninidhud akọ vuh nanjik, Nunh nikyọ tinjih nirzịnut kwuh tsuh chozi; Nekuddi ttrin zit kendo ttrin zit tthunkit kwu ttsun ninechin, zyeh zyo nethizi, 
Akọ Vittukoochanchyo Kwittyi Thlanchi ha nichinttai nandei tsei nyoti: Azyuk kwu ttsun kunutetik kwundaikthut nininchikkthut ha untoututatri kenjit.

Kwikyinjihshit Chunkyọ Rsotitinyoo koọli ; Thlachya Rsotitinyookthut nikyo kootukookwinyachyo zyunkoọli ; trigichinchilk nichya zyunkwitili ; Troogwandyoh ekootichya; kwizyin nunukwutalye, Akọ sheggu kwundai. Âmen.

I Azyuk kootle chih kwigichinchi akọtigwititetinja, tutthug nuntshikwuttrule: Gikhyi trootshit akọtititenja kwitezyoo nichinttai $h a$ :

Kukwadhud nuwhah kolị.

Kwiti-kwigikhyi. Akọ nyuh nkyọ ha. Gikhyi. Zyunguttochya.

Kukwạdhud, nersingichachyo tunnuwhohchịnyoo.

Christ, nersingichachyo tunnuwhohchịnyoo. Kukwạdhud, nersingichachyo tunnuwhohchịnyoo.

I Azyuk kootle Gikhyi, tinjihnut ha kukwadhud kwigikhyi kwitezyoo nichinttai ha akotungititenja.

WUWHOH Ttyi zyeh zit nih kwilnjik. IN Nyooh rzi chootsi. Nih kookukwadhud nu vizi. Tinịdhun nunh kuggu tugoochyo nittso zyeh zit troogwattsi. Chih ttrin zit nuwhun enyantsit ttrin ndo thloogootunazya nuwhoh ssih. Nuwhot troogwandyoh nuwheh unkoochịli nittso unkoochitili einut troogwandyoh 6 
VUNTAI TRIGIKHII.

nuwheh tungitiyin. Kookukwundai kwu tsut nuwhoh tunöe rshọ: kọ troogwandyoh $\mathrm{kwu}$ itsut nuwhoh yunninji. Amẹn.

THE COLLECT FOR GRACE.

Kwigichinchi chetrsikoonchyo kenjit.

KUKWADHUD nuwhoh Ttyi zyeh kug, Thlanchi ha nichinttai ako sheggu Viteukoochanchyo tinichyo, nunnuwhoh nili chooggoo ttrin nugwitili kwu tsut; Chih ttrin zit nuwhoh kunnyatyi nyih ttuih ehoh ha; ako tunnuwhoh chinyoo chooggoo ttrin troogwandyoh kwinjyo elyet kwiyuntudhatthah, ako elyet zyuntudhakul ekoochichyo koogwunachut kwinjyo; ko kwuttutthug nuwhoh koottrid nih kinjik kit tunitiyin chi, rsittuttsut nyih ndah sheg akọtuntitiya; nuwheh kukwadhud Jesus Christ kirre. Amen.

A PRAYER FOR ALL CONDITIONS OF MEN.

Krwigichini tinjih nittso tsut koohzyunkwilnjik enjit.

VITTUKOOCHANCHYO, tinjih tutthug
zyunkoohahantsei, ako kooh kunnyatyi, chettukotithinyoo ha nih tsut zyungittinichi tinjih tutthug nithlinetsi tunthichyo ako nittso tsut kooh zyunkwilnjik: sso ịlị chih nyih nutinde kazyunkootandul, nih chassi tutthug kutchinut tsut. Treshit tsut nih tsut ryungittinichi trigichinchi tatthug kwirzi zyungittelyah: Chunkyo Rsotitinyoo kwikit zyunkoohtuneä ako kooh tsuh kunkwutadhud, akẹtukoonchyo tutthug Christ ttsun gịli zyunuttugutatinyoo 
thliëtetli kwundo zyunkoohtunetuä, akọ kwikyinjigwizhit zyunkotetunh chihthloog kwunkyọ ha, ttsinettun ha, akọ tshoogoochoë kookwundai ha. Huịko tsut kwittyi nyeh rsikoonchyo nih tsut zyungittinichi huịnjikidichinut enjit, kwaütseịnugwichyanut ha kwiyinji, akọ nittsọ tsut koohzyunkwilnjik ha : sso ịli chi khukohtạntah akọ kooh tsuh truntịndai nittsọ kettun zyungittili, tạthoog huịjikidichi kwukhetachyo koohnittlunchịe, akọ kooh huịnjikwidichi kwu ttsun sso koọli ha kwitshilikwutedha, Chih yoo zyunochitukthut Jesus Christ kirre. Amen.

A GENERAL THANKSGIVING.

\section{Tutthug kenjit hui kwuntlukwitäei.}

ITTUKOOCHANCHYO Thlanchi ha ni-
chinttai tinicho, chetrsikoonchyo kwuttutthug Kwittyi ịli, nuwhun nyih ultsunnut elyet kunitili etchunetotidhitinyoo, huị nyih ntlunchitaë nuwhoh ttri ha kwuttutthug nih chetrsikoonchyo akọ nih chettigwinidhun kenjit nuwhoh tsut ako tinjih tutthug tsut. Huị, nih ntlunchitaë nuwhoh dhantsei, nuwhoh kunnyidhantyi, kwuttutthug ekoochichyo kwịrzi ha chih kwundai zit; ko kwiyendo tsut nih chettigwinidhun chịnttsi tinjih zyunnyạsi nuwheh Kuwadhud Jesus Christ kirre ; chetrsikoonchyo kwutsuhtrigwinji kenjit ako nindelh kunvitili. Ako nih tsut zyungittinichi nuwhot ttri zit huị tuntutunaä kwuttutthug nih zyooggutdichi kenjit, azyuk $\mathrm{kwuggut}$ nih tettichile kookunuttunatya, nuwhoh tevah rai ha ukkwa, ko kwuttutthug 
nuwhoh kwundai ha; nyih ntletututazya, nyih kukwutatah, ako nyih ndah kwutsuh tituta rsikotitinyoo akọ tshoogoochoë tsut kwuttutthug nuwhot ttrin zit; nuwheh Kukwadhud Jesus Christ kirre, yaggun tsut, akọ nih tsut, akọ Chunkyọ Rsotitinyoo tsut kwuttutthug chịnttsi ako nindelh tugoochyo sheggu kenjit akọ sheggu kenjit. Amen.

A PRAYER OF ST. CHRYSOSTOM.

Chrysostom rsotitinyoo kwigikhyi.

VITTUKOOCHANCHYO thlanichi ha nichinttai tinichyo, chetrsikoonchyo nuwhoh ntlunchidhịli chihthloog $\mathrm{k}$ wittri ha chunzyunnithlichazi nih tsut zyungittinichi kenjit; akọ kheniteitinịtäeị etchittaị nekunnut ko ttyẹnut thlugachya nyooh rzi zit kooh nittlutilya chitti tte zyunkotutukthut: 0 Kukwạdhud chooggoo zyunodhudhantshei nih ultsunnut kooh tinjikwidichi, akọ kooh zyungichinchi nittso kwenjit kwiyendo tsut kwiterzyah : chih nunh kug nuwhoh ntluntechịli nih thliëtetli kazyuntitundai akọ nunh kug nukootutattha sheggu kwundai. Amen.

\section{Cor. XIII.}

NUWHEH Kukwadhud Jesus Christ vih 1 chetrsikoonchyo, ako Vittukoochanyo vih chettigwinidhum, ako Chunkyo Rsotitinyoo nichya sheggu nuwhah tungoochyo. Amen. 
THE

ORDER FOR EVENING PRAYER DAILY THROUGHOUT THE YEAR.

\author{
mm n
}

KHAH TSUT TRIGITKYI NUKWUTUTHUD TUTTHUG KENJIT.

\title{
mmon
}

HTCHITAI tinjih trandyoh ei troogwan1 dyoh tetiyin akhekonyoo, akọ ei rsittutsut ako tshoogoochöe tsut titiyin tuh nkyo tassi. Ezek. xviii. 27.

Suh troogwandyoh kwulhnduk, akọ suh troogwandyoh suh ndah sheg kooeị. Ps. li. 3.

Suh troogwandyoh kwu ttsut nih nyun ninyai, ako tutthug suh troogwandyoh oounkoochịli. Ps. li. 9.

Kineinjitowhohchi : kwugguh yoo zyeh kug kwu ttsut kookudwadhud tsuh nyakwuth. St. Matt. iii. 2.

Nutihseh akọ ttiạ vuh tsuh tihsyah, akọ akotuvutakhnja: Ttiạ zyeh zit kwu tsut ako nyih ndah troogwandyoh kwudhelhtsei : akọ tthui tte elyet giihthlị sih tinji suh tạja. St. Luke xv. 18, 19.

Elyet troogwandyoh kundhiti zyunchitinyoo chi, zyunnettitinitaë, akọ elyet thliëteg10 
witli nuwhoh zit kwili. Nuwhoh troogwandyoh keh zyunkwanduk chi, thlih ha ako tshitchoë nilị nuwhoh troogwandyoh nuwheh ooun kootelya, ako tutthug troogwandyoh kwu ttsut nuwhun uttata. 1 John i. 8,9.

K WINTLUTH nuwhet tunyinutudhun Il kwuchanut, Etunetle choh kwi zit nikhun kwitekit akotunnuhwhahnyoo nuwhoh troogwandyoh kwilyei zyunkwutanduk : ako elyet tunnyinudhaä Vittukoochanchyo thlạchi ha nichinttai zyeh kug nuwhoh Ttyi vuh ndah : ko kwittri chettukotithinyoo, thlitseyinjikwitunzhit, ako kookukwathut ha; ei kwu tsut nuwhoh troogwandyoh ekooteta, vih chetrsikoonchyo ako vih rsinichi ha kirre. Akọ Vittukoochanchyo ndah chettukotithinyoo ha nuwhoh troogwandyoh sheg zyunkwanduk chi ; kwuttuzug kwiyendo tsut akotunitiyin chi, etchittaị chunzyunnithlichazi huị vuh ntlunchitaë tutthug vih chehrsikoonchyo kenjit, vih chile akọtuntanja, akọ zyunotutakhut tutthug ei ekoochichyo tinjikwitizhit kwizyin akọ kwunkyọ enjit. Azyuk kwuggut akọtunnuwhohchikhnyoo nuwhot tutthug tzet zyunohthli sih kit teigokhyah kwittri rsittuttsut ako kwitezyoo chettukotithinyoo ha zyeh kug tetletchiti chetrsikoonchyo kwu tsut, akọtunchitinyoo:

II Kehkwanduk thlachya tutthug akotungititenja Gidhyi ha, tutthug nuntshitcwuttrule.

THLACHI ha nichinttai tinichyo akọ kwitI tyi kwintluth nyetrsikoonchyo: Nyih 11 
netinde kwuttsun thlettinidhidichil, akọ tettinidhidichil tivi hazyuntrookwetunduk kit. Nittso nuwhot ttri zit nunyinjidhidichik ako tunyinjidhidıchik treshit kwikit tedhidichil. Iih kinjik rsotitinyoo tsut koottsite zyunkwudhatseị. Elyet akọtungwaya ei akọtungwayin chi: akọtungwayin ei elyet akọtungwaya chi: akọ nuwhoh zit kwịrzị kookwundai kwilị. Kọ nun Kukwạdhud nersigoochachyo tunnuwhoh chịnyoo huịjiidichi troogwandyoh akọtungwayịn. O Vittukoochanchyo rsyunkoohchanchyo einut kuttuh troogwandyoh nyih ndah keh zyunkookwanduk. Chun kwettunyinịdhun nih tsuh kinugutta; nyih khenitteikwichittäei kit tinjih tsut nuwheh Kukwadhud Jesus Christ kirre. Akọ Kwittyi kwintluth nyetrsikoonchyo, vih kirre akotunnuwhohchịnyoo; choog kwu ttsut shọ elyet zyunkwutitundai nih kinjik kit, tshoogoochöe tsut, akọ rsittuttsut, nindelh tsut nyooh rzi rsotitinyoo. Amen.

\section{ब Troogwandyoh ekoodichya, Gizhyi rzi nu- dhut alotititenja; tinjih tthuih tte nuntshi- kwuttrule.}

THLANCHI ha nichinttai Vittukoochan1 chyo, Kwittyi nuwheh Kukwadhud Jesus Christ, ei yoo elyet akọtinidhun ihthlẹ trandyoh nutedha, Ko kwiyendo tsut yih troogwandyoh akhekotitenja ako kwutendai : ako kwuttai akọ kettugwichiltshei tih gịkyinut kwuntlutetäei, akọtungititenja akọ kunkwutanduk tih tsyahnut kooh troogwandyoh akhekotitinyoo: Ei yoo tutthug einut thlih 
ha thlitsetunyinjigichi kooh troogwandyoh oounkootili. Azyuk kwuggut vuh tsuh zyungittinichi thlih ha thlitseyinjikwitizhit nuwhoh ntlutetua, ako vuh Nkyọ Rsotitinyoo, ei ekoochichyo choog tunitiyin sso vuh tsun yih tatsya, ako choog kwu ttsut nuwhoh kwundai rsikotitinyoo ako kwịrzi kwitelya: akọ tukoonchyo huiko tsut sheggu vih ssokoọli nutituta; Jesus Christ nuwheh Kukwadhud kirre. Amen.

9 Tzet ako tutthug trigikhyi kwittsi, tinji akotuingititenja. Amen.

बा Ako tle Gikhyi nitshikwuttuttaë, ako Kukwadhud kwigihyi ahotititenja; tinji thuị nuntshikwuttrule, gih kit teikigikhyi.

NUWHOT Ttyi zyeh zit nih kwilnjik. 1 Nyooh rzi chootsi. Nih kookukwadhud nuvizi. Tinịdhun, nunh kuggu tugoochyo nittso zyeh zit troogwatsi. Chih ttrin zit nuwhun enyantsit ttrin ndo thloogootunazya nuwhoh ssih. Nuwhot troogwandyoh nuwheh unkoochịli nittso unkoochitili einut troogwán. dyoh nuwheh tungitiyin. Kookut-kukwadhud kwa tsut nuwhoh tunoe rshọ: ko troogwandyoh kwu ttsut nuwhoh yunninji. Kwugguh yoo nih ttsun nili kookukwadhud, ttai, ako nindelh sheggu kenjit akọ sheggu kenjit. Amen.

Nunc dimittis. St. Luke ii. 29.

K UKWADHUD chooggoo nih ultșun kethlechoozi nih kinjik kit. 13 

yin :

Kwugguh yoo suh nde nyih chassi kwunạ-

Ei yoo tinjih tutthug ndah rsyenukwiniei ;

Attri atshinkutchin khuạttri, akọ, nih tsyahnut Israel kooh chịttsi.

Kwittyi akọ kwitinji : akọ Chunkyo Rsotitinyoo ha chịttsi kolị ;

Nittso koogwinyathud kwịlị, chooggoo koọli, akọ sheggu kenjit kwittelya: nunh kwilikwutetha kwu tsut. Amen.

ๆ Akọtle Chilehnut kooh kwikyinjigwizhit akotititetinja Gibhyitinjihnut ha nugichilzi kirre.

TW WIKYINJIHSHIT Vittukoochanchyo 1 kooli kwittyi Thlanchi ha nichinttai, yzeh nunh ha eltsei :

Akọ ei rzị vih Tinji Jesus Christ nuwheh Kukwadhud, Ei Chunkyọ Rsotitinyoo kirre tinjih ulttsei Nichid Mary ttsut vih kwịlị, Pontius Pilate teggug huịnjithudhut, Tuchun kwinititä̈ kug gulttsuk, ninidhud akọ vuh nanjik, Nunh nikyo tinjih nirzịnut kwuh tsuh chozi; Nekuddi ttrin zit kendo ttrin zit tthunkit kwu ttsun ninechin, zyeh zyọ nethizi, Ako Vittukoochanchyo Kwittyi Thlanchi ha nichinttai nandei tsei nyoti: Azyuk kwu ttsun kunutetik kwundaikthut nininchikkthut ha untoututatri kenjit.

Kwikyinjihshit Chunkyo Rsotitinyoo koolị; Thlachya Rsotitinyookthut nikyo kootukookwinyachyo zyunkoọlị; trigichinchik nichya zyunkwitilị; Troogwandyoh ekootichya; kwizyin nunukwutalye, Akọ sheggu kwundai. Amen. 
KHAH TSUT TRIGIKHYI.

ๆ Azyuk kootle chih kwigichinchi akotigwititetinja, tutthug nuntshikwuttrule: Gikhyi trootshit akọtititenja kwitezyoo nichinttai ha:

Kukwadhud nuwhah koli.

Kwiti-kwigikhyi. Akọ nyuh nkyo ha.

Gikhyi Zyunguttochya.

Kukwạdhud, nersingichachyo tunnuwhohchịnyoo.

Christ, nersingichachyo tunnuwhohchịnyoo. Kukwạdhud, nersingichachyo tunnuwhohchịnyoo.

(T Azyuk kootle Gikhyi, tinjihnut ha kukwadhud kwigikhyi kwitezyoo nichinttai ha akotungititenja.

NUWHOH Ttyi zyeh zit nih kwilnjik. 1 Nyooh rzi chootsi. Nih kookukwadhudnuvizi. Tinịdhun nunh kuggu tugoochyo nittsọ zyeh zit troogwattsi. Chih ttrin zit nuwhun enyantșit ttrin ndo thloogootunazya nuwhoh ssih. Nuwhot troogwandyoh nuwheh unkoochịli nittso unkoochitili einut troogwandyoh nuwheh tungitiyin. Kookukwundai kwu tsut nuwhoh tunöe rsho: kọ troogwandyoh kwu ttsut nuwhoh yunninji. Amen.

THE COLLECT FOR AID AGAINST ALI PERILS.

Kwigichinchi kwutsuhtrigwinji kenjit tutthug koogwunachut kwu tsut.

KUKWADHUD nuwhoh tsegga anttri nih tsut zyungittinichi; akọ nih zyoo15 


\section{KHAH TSUT TRIGIKHYI.}

ggutdichi kirre koogwunachut unduihkwichanchyo ha kwuttutthug kwu ttsun nuwhoh kunnyatyi chooggoo ttodh; chihthloog rzi nih Tinji Jesus Christ nuwhoh Assi vih chettigwinidhun kirre. Amen.

A PRAYER FOR ALI CONDITIONS OF MEN.

Kwigichini tinjih nittso tsut koohzyunkwilnjik enjit.

VITTUKOOCHANCHYO, tinjih tutthug zyunkoohdhantsei, ako kooh kunnyatyi chettukotithinyoo ha nih tsut zyungittinichi tinjih tutthug nithlinetsi tunthichyo akọ nittsọ tsut kooh zyunkwilnjik: sso ịi chih nyih nutinde kazyunkootandul, nih chassi tutthug kutchinut tsut. Treshit tsut nih tsut zyungittinichi trigichinchi tutthug kwịrzi zyungittelyah : Chunkyọ Rsotitinyoo kwikit zyunkoohtuneä akọ kooh tsuh kunkwutadhud, akọtukoonchyo tutthug Christ ttsun gịlị zyunuttugutatinyoo thliëtetli kwundo zyunkoohtunetuä, akọ kwikyinjigwizhit zyunkotetunh chihthloog kwunkyọ ha, ttsinettun ha, akọ tshoogoochoë kookwundai ha. Huịko tsut kwittyi nyeh rsikoonchyo nih tsut zyungittinichi huịujikidichinut enjit, kwaütseịnugwichyanut ha kwiyinji, akọ nittsọ tsut koohzyunkwilnjik ha: sso ịli chi khukohtạtah akọ kooh tsuh truntịndai nittsọ kettun zyungittilị, tạthoog huịjikidichi kwukhetachyo koohnittlunchịe, akọ kooh huịnjikwidichi kwu ttsun sso koọlị ha kwitshilikwutedha. Chih yoo zyunochitukthut Jesus Christ kirre. Amen. 
A GENERAL THANKSGIVING.

\section{Tutthug kenjit hui kwuntlukwitäei.}

JITTUKOOCHANCHYO Thlanchi ha nichinttai tinichyo, chetrsikoonchyo kwuttutthug Kwittyi ili, nuwhun nyih ultsunnut elyet kunitili etchunetotidhitinyoo, huị nyih ntlunchitaë nuwhoh ttri ha kwuttutthug nih chetrsikoonchyo ako nih chettigwinidhun kenjit nuwhoh tsut akọ tinjih tutthug tsut. Huị nih ntlunchitä̈ nuwhoh dhantsei, nuwhoh kunnyidhantyị, kwuttutthug ekoochichyo kwịrzi ha chih kwundai zit; kọ kwiyendo tsut nih chettigwinidhun chịttsi tinjih zyunnyasi nuwheh Kukwadhud Jesus Christ kirre: chetrsikoonchyo kwutsuhtrigwinji kenjit ako nindelh kunvitili. Ako nih tsut zyungittinichi nuwhot ttri zit hui tuntutunaä kwuttutthug nih zyooggutdichi kenjit, azyuk kwuggut nih tettichile kookunuttunatya, nuwhoh tevah rzi ha ukkwa, ko kwuttutthug nuwhoh kwundai ha; nyih ntletututazya, nyih kukwutatah, akọ nyih ndah kwutsuh tituta rsikotitinyoo ako tshoogoochoë tsut kwuttutthug nuwhot tirin zit; nuwheh Kukwadhud Jesus Christ kirre, yaggun tsut, ako nih tsut, ako Chunkyo Rsotitinyoo tsut kwuttutthug chịttsi akọ nindelh tugoochyo sheggu kenjit akọ sheggu kenjit. Amen. 
A PRAYER OF ST. CHRYSOSTOM.

Chrysostom rsotitinyoo kwigikhyi.

TITTUKOOCHANCHYO thlạnchi ha nichinttai tinichyo, chetrsikoonchyo nuwhoh ntlunchidhịli chihthloogkwittri ha chunzyunnithlichazi nih tsut zyungittinichi kenjit; akọ kheniteitinịtäeị etchittaị nekunnut kọ ttyenut thlugachya nyooh rzi zit kooh nittlutịlya chitti tte zyunkotutukthut: O Kukwạdhud chooggoo zyunodhudhantshei nih ultsunnut kooh tinjikwidichi, akọ kooh zyungichinchi nittso kwenjit kwiyendo tsut kwiterzyah : chih nunh kug nuwhoh ntluntechịli nih thliëtetli kazyuntitundai akọ nunh kug nukootutattha sheggu kwundai. Amẹn.

\section{Cor. xiii.}

NUWHEH Kukwadhud Jesus Christ vih 1 chetrsikoonchyo, ako Vittukoochanchyo vih chettigwinidhun,akọ Chunkyọ Rsotitinyoo nichya sheggu nuwhah tungoochyo. Amen. 


\section{PRAYERS. \\ (KWIGICHINCHI.)}

FIRST SUNDAY AFTER EPIPHANY.

KUKWẠDHUD nih tsut zyungittinichi nih chetrsikoonchyo kirre nih tsyahnut nyoọkkunkigịkhyi kooh zyungichinchi tukwạnji ; akọtunkoochịnyoo kunyinjigitteyin akọ kazyunguttetundai chitti tte kwikit tungitettiyah, ako kettungittinezhya akọ nungututettai rsittuttsut akọtungitenjah; nuwheh Kukwadhud Jesus Christ kirre. Amen.

SIXTH SUNDAY AFTER TRINITY.

VITTUKOOCHANCHYO einut nyettunginidthun enjit kihrsyenugwinyạei ekoohichyo kwirrzi tinjih kwikittutzigwele $\mathrm{kwi}$ yendo tsut kwuttoyinjikwitizhit : Nuwhoh ttri zit nih chettigwinidhun ha tuntinịli kwuttuthugekoochichyo kwiyendo tsut nyet tunyinitudhun chi nih kheniteikwichitäei kuntitiyah, ei yoo kwuttutthug tunyinjidichi kwiyendo tsut kwichịnttsi ; nuwheh Kukwadhud Jesus Christ kirre. Amen.

* a prayer for a sick person.

Kwigichinchi ei eltsik enjit.

VITTUKOOCHANCHYO thlanchi ha nichinttai, tinichyo, ako Kwittyi nyeh rsikoonchyo kwundai etshị ha chutsut kukwạ-

* From Order for Visitation of the Sick. 
dhud: Zyeh zit kwutto nyayin chih tinitye nih zyooggutdichi ha nih tsut zynngittinichi. Nih chassi ha veh niniheih, O Kukwạdhud; vih zyin khursanië kwu ttsut vih yininji, ako vuh nkyọ nyasi nih zyooggutdichi kirre. Sso ịlị, chi chih nunh kug vih ttrin kwinjyo kwutantsya. nih tsut kwuttendai rsittuttsut nih kutanduk, akọ kwịrzi kwikit titettiya; elyet kwikit tittechya chi, ei zyeh kug kootekit kwizit oninji nichin tte kwunkyọ einut Kukwạdhud Jesus zit gelchyuth sheggu nukwazi ako ssokoọlị zyungaị. Kwikit tinịdhun, O Kukwadhud, nih zyooggutdichi kirre nih Tinji Jesus Christ nuwheh Kukwadud kirre, ei nyah kwundai ako nyah Kudwadhud nun akọ Chunkyọ Rsotitinyoo sheggu chihthloog Vittukoochanchyo ịli nunh kwilikwutedha kkwa kwu tsut. Amen.

THE ORDER OF THE

ADMINISTRATION OF THE LORD'S SUPPER.

KUKWADHUT KWI KHAH TSUT CHUTRUA RSIKOTITINYOO, NITSO KWUNTLUKWITAEI.

Kukwadhut Kwigichinchi.

UWHOH Ttyi zyeh zitnih kwilnjik. Nyooh I rzi chootsi. Nih kookukwadhud nevizi. Tinịdhun nunh kuggu tugoochyo nittsọ zyeh zit troogwattsi. Chih ttrin zit nuwhun enyantsit ttrin ndo thloogootunazya nuwhoh ssih. 20 
Nuwhoh troogwandyoh nuwheh oounkoochịli nittso oounkoochitili einut tsut troogwandyoh nuwheh tungittiyin. Kookukwundai kwu tsut nuwhoh tunọë rshọ: kọ troogwandyoh kwu ttsut nuwhoh yunninji. Kwugguh yoo nih ttsun nilị kookukwadhud, ttai, akọ nindelh sheggu kenjit akọ sheggu kenjit. Ámen.

\section{Kwigichinchi.}

\section{VITTUKOOCHANCHYO thlanchi ha} nichinttai tinichyo, nih tsut kwittri tutthug nikinichya, koọuttigwinidhun tutthug kakwitundai, ako nih tsut ekoochichyo elyet ninilii : nuwhoh ttri ttsut kwiyinji unchanchye nih Chunkyo Rsotitinyoo tezi kirre, thlanchi ha nyet tunyitinitizhya, akọ nyooh rzi tsenjah zyuntututatsi, nuwheh Kukwadhud Jesus Christ kirre. Amen.

I Azyuk kootle Gikhyi Chihthloogchotyin tugwitetuggun akotititenja kwirzye tsut : ako tinjih tthui nuntshikwuttrule, tutthug tugwitie tle Vittukoochanchyo tsuh zyunkigititechya kooh troogwandyoh unkootelyah kenjit, ako yendo kwundo kooh tsuh truntendul, ei yoo kunkutunatya.

Gikhyi.

JITTUKOOCHANCHYO chih kinji ha gikhe: Kukwadhud nih Vittukoochanchyo ihthlị :

Elyet chizi Vittukoochanchyo vih tudhintiya sih rzi.

Tinjih Kukwadhud nersingichachyo tunnuwhohchịnyoo, akọ nuwhoh ttri zyunnịa chih tugwittië kookuntunnatya. 
Elyet ekoochichyo kwikittelttsi tutudhan. tsya, elyet ekoochichyo yette zyeh zit kwikit tigwichyo, nunh kuggu zuggudhud, choọ zit nunh yezyuggu thui. Elyet kooh ndah nitshikwuttutudhạdhud, ako elyet kooh tsuh gittitidhinchya: Kwugguh yoo sih, Kukwadhud nih Vittukoochanchyo, Vittukoochanchyo kwantrhị ihthlị, akọ kwittyinut kooh troogwandyoh kwikkyinut tsut nunkootulhah ei tsut ttyid tank thut nizyoọnedichi einut truttun suhgoochachyo; akọ chetrsikoonchyo kunonulhtun chihthloog tinili kit chotyin tsut chotyinut einut set tunginidhun, akọ sih tugwitetuggun kunkunatye.

Tinjih. Kukwạdhud nersinginchachyo tunnuwhohchịnyoo, akọ nuwhoh ttri zyunnịa chih tugwittịe kookuntunnatya.

Kukwadhud nyeh Vittukoochanchyo vooh rzi zyọ ọnji rshọ: kwugguh yoo ei yooh rzi zyọ oonji troogwandyoh kwatsi kkwa Kukwadhud yoohtittenja.

Tinjih. Kukwadhud nersingichachyo tunnuwhoh chịnyoo, akọ nuwhoh ttri zyunnịa chih tugwittịë kookuntunnatya.

Nukwazi ttrin kununyandai rsikotitinyoo kookutunantya kenjit. Nikyittyid ttrin zit koottrid tugswutạya, akọ chitti koottrid kunitiyin kwikit titintiya : ko nikittyid ttrin zit kendo ttrinzit nukwazi ttrin zit koọlị Kukwadhud nyeh Vittukoochanchyo ttsun nili. Ei ttrin zit elyet ekoochichyo koottrid tug: witutudhayah, nun ako nih tinji, nih ttshị ha, tinjih nyih kanduk, ttrig nyih kanduk ha, nih chutthuị, akọ atshin kwizit nitugwiti. Kwugguh yoo nikittyid ttrin zit Kukwadhud 22 
zyeh nunh ha eltsuị, choochoh tutthug kwizit koọli ha, akọ nikittyid ttrin zit kendo ttrin zit nazi : azyuk kwuggut nikittyid ttrin zit kendo ttrin zit Kukwadhud kwịrzi kwuh tsuh gịkhe, akọ rsikotitinyoo kweltseị.

Tinjih. Kukwạdhud nersingichachyo tunnuwhohchịnyoọ, akọ nuwhoh ttri zyunnịa chih tugwittie kookuntunnatya.

Nih ttyi nuyuh hun ha yinjikoohchidhä̈: ei kthoikit kwizit Kukwadhud nyeh Vittukoochanchyo nih ntlukwüei, nih ttrin zit kwutenjyo.

Tinjih. Kuḳwạdhud nersingichachyo tunnuwhohchinyoo, ako nuwhoh ttri zyunnia chih tugwittië kookuntunnatya.

Tinjih dhọkthah rshọ.

Tinjih. Kukwạdhud nersingichachyo tunnuwhohchịnyoo, akọ nuwhoh ttri zyunnịa chih tugwittië kookuntunnatya.

Tseyinọzhin rshọ.

Tinjih. Kukwadhud nersingichachyo tunnuwhohchịnyoo, akọ nuwhoh ttri zyumnịa chih tugwittịe kookuntunnatya.

Chunọi rshọ.

Tinjih. Kukwạdhud nersingichachyo tun. nuwhohchịnyoo, akọ nuwhoh ttri zyunnịa chih tugwittịe kookuntunnatya.

Nyih neäggoo vọtsit rsho.

Tinjih. Kukwạdhud nersingichachyo tunnuwhohchịnyoo, ako nuwhoh ttri zyunnịa chih tugwittië kookuntunnatya.

Nyih neäggoo vih zze kwu tsuh tinjotizhit rsho, nyih neaggoo trenjo tsuh tinjọtizhit rshọ, tinjih yih kanduk, trig yih kanduk ha 23 
vuh aki tthui, vih thlui choh utze choh, ekoochichyo vuh ttsun koọlị tthui.

Tinjih. Kukwạdhud, nersingichachyo tunnuwhohchịnyoo, ako kwuttutthug chih nyih tugwitetuggun nuwhot ttri zit zyunkwittinintluth nih tsut zyungittinichi.

\section{ब Azyuk kootle chihthlud chih kwigichinchi akọtititetinja Kwiyäutnjo enjit, gịkhyi nud- hut akotititenja, \\ Zyunguttochya,}

THLLANCHI ha nichinttai. Vittukoochan1 chyo, nih kookukwadhud sheggu koọli, akọ nyih ttai kwilinkootizhit kkwa: 'Trigichinchi thlachya zyooggutdichi ha tunkoohchịnyoo; akọ nih Ultsun uttotitinyoo $V I C$ $T \dot{O} I A$, nuwhoh kwiyeutnjo akọ nuwheh Kukwadhud vih ttri kwikit kukwadhud, (chootyin ultsun katundai) tutthug ekoochichyo kwiyendo tsut nih yinjikwichile ako nih chịnttsi koọkkuttunetya: akọ nuwhun, tuthug vih tsyahnut ha, (tsenja nunyinjidichi chootyin kookukwadhud tiyin), kwịzị tsut vih kuntututanduk, zyunyinjitututaä, akọ huzuggutidichyo ha vih kunkwutata, nih zit akọ nyeh njit, nih kinjik nirzị akọ nih kuttukwitadhud kit: nuwheh Kukwadhud Jesus Christ kirre, ei nun ha akọ Chunkyo Rsotitinyoo ha kwundai ako kudwadhud, sheggu chihthloog Vittukoochanchyo ịi nunh kwilikwutedha kkwa kwu tsut. Amen.

\section{Ko chih.}

THLANCHI ha nichinttai ako sheggu 1 Vittukoochanchyo, nih etunetle rsotitin24 
yoo kirre kunnuwhohnatun, kukwadhudkthut kooh ttri zyunnịa akọ kwu tsuh kukwạdhud, akọ nunyinjikoochịë akọ uttsetinịlië nittsọ kwiyendo tsut kwịrzi nih kwizzi kwu tsut: Chettukotithinyoo ha nih tsut zyungittinichi VICTORIA nih ultsun nuwhoh kwiyëut njo, ako nuwheh kukwadhud vih ttri kwikit ninjikootiniei ako kwu tsuh kukwạdhud, vih yinji tutthug, vih kinjik, vih koottrid ha, nih yinjikwichile ako nih chịnttsi sheg kookuttunetya, ako ninjitedhut nih tsyahnut kih nili zit nunkoohtaë kookuntunatya kwichile ha, tsinettun ha, akọ nih kinjik kit: Akotochịnyoo. K.wittyi zyoogguttinidichyo, nih 'Tinji vet tinuthun kirre, nuwheh Kukwadhud Jesus Christ. Amen.

THE NICENE CREED.

\section{Azyuk kootle chih kwikyinjigwizhit akọtititetinja.}

K WIKYINJIHSHIT Vittukoochanchyo chihthloog kooli, kwittyi thlanchi ha nichinttai, zyeh nunh ha eltseị. Akọ ekoochichyo tutthug kwunayin ako elyet kwiniliyin :

Ako Kukwadhud ihthle Jesus Christ. Vittukoochanchyo vih Tinji chihthloog rzị, vih Ttyi ttsut vih kwịlị nunh kwuttutthug kwiyetshi tai, Vittukoochanchyo ttsut Vittukoochanchyo nili, Attri ttsut Attri, Thlih ha Vittukoochanchyo thlih ha Vittukoochanchyo ttsut, Vih kwilịh elyet Attsya, Kwittyi ha chihthlug ttsun nili, Ei kirre ekoochichyo tutthug kwulttsei : Ei nuwheh njit, tinjihnut zyunitili, ako nuwhoh chassi kenjit, zyeh kug kwu ttsun neịzye. Akọ kwutthuị dhitlit Chunkyo 
Rsotitinyoo kirre nichid Mary ttsut. Ako tinjih ulttsei, Akọ gulttsuk ei thui nuwheh njit. Pontius Pilate teggug. Huịjithudhut ako vuh nanjik, Akọ nekuddi ttrin zit kendo ttrin zit, thunkit kwu ttsun ninechin attunututluth kit. Ako Kwittyi nandei tsei nyoti. Ako nindelh ha kunutetik kwundaikthut nininchikkthut ha untoututatri kenjit: Vih kookukwadhud kwilikwntedha kkwa.

Akọ Chunkyọ Rsotitinyoo koọli kwikyinjihshit. Kwundai kwu tsuh Kukwadhud ako kwuntlukootäei. Ei Kwittyi Kwitinji ttsun ha niheih. Ei Kwittyi $K$ witinji ha vuh tsuh trigichinchi akọ vuh tsuh tutichile. Ei kuttugwitäugkthut kirre gịkhe. Akọ kwikyinjihshit chihthloog thlachya nikyo kootekookwinyacho, ako thlictegwitli. Chihthloog kwinenyitinjah troogwandyoh ekootichya kenjit kwikit kahsundai. Ako nininchikkthut nunnukootalye kukovihthli. Akọ nunh nukotutattha kookwundai. Amen.

Tा Tuhkwanduk tle Gikhyi Kuhwadhud vikugchï̈ tsuh tahah, ako ihthlud ko kwiyendo lyei chih vikuazye tsul akotititenja, nittso tsut kwirzi teyitinezhya.

TUWHOH attri tinjih ndah vattri, nuwhoh njit, ako nuwhoh Ttyi zyeh zit nili zyunguttutasi. St. Matt. v.

Nunh kug tette ukohzye rshọ; nichin kwitittrhyo akọ kooka rsyetetkoogootathlye, akọ nichin chunikthut thlunkootanei akọ zyunchuni : ko zyeh zit tette ukwudhohzye; nichin tte kwitittrhyo ako kookạ rsyetetkoogoo26 
tilthlye, akọ nichin chunikthut thlunkootulhnei akọ zyunchuni kkwal \$t. MLatt. vi.

Chitti tte tinjiwhohchi tinjih nuwheh njit kwikit tungittetiya, kwikit tukoonchyo kooh tsuh tunwhohyin: kwugguh yoo chih yoo Tugwittịe akọ kuttugwitaugkthut. St. Matt. vii.

Elyet titugookwinyachyo akọtsusahnyoo, Kukwạdhud, Kukwạdhud, zyeh kug kookukwadhud nitte tehah : kọ eị Ttiya zyeh zit nilị nih nyun kit titiyin. St. $\dot{M} a t t$. vii.

Zacchæus Kwiyëudh nudhut, akọ Kukwadhud tsut akọtitinyoo: Kukwạdhud kwinyạyin, suh kwuttul tenjirh tsut nersichachyonut kooh nittluihthli: akọ tinjih ihthle tsut teko tihsiyin chi tankthut koogwantsi tsut vuh ntlekwilhnji. St. John, xix.

Chootyin nukoove kwuh tsuh taheih attun thun etti kirre? Chootyin chug choh kwunzi kwauntutthui, akọ yih chug elyet eä? Ako chootyin tivi unaä, ako yih ttok elyet thinyin? 1 Cor. ix.

Kwunkyọ enjit ekoochichyo nuwhoh tsut nunyitunja chi lyet kwịlẹ nunh kug nuwhoh echichi nunkwutunatsya. 1 Cor. xi.

Elyet lyeh kazyundhohwhunjik, einut ekoochichyo rsikotitinyoo kenjit kukunkanduk, tshihtakyi ha zyunkookwundai; ako einut vuh kug tshihchikyi nunkohlii, tshihchikyi ha zyunkoonjih? Kwikit tukoon chyo tthui Kukwadhud kuttukoochildhud, einut kwunduk nirzi zyunkookwanduk, kwunduk nirzị kirre zyunkookwundai. 1 Cor. ix.

Ei kwintsul nukwili kwintsul hugwitetul : akọ ei kwịlyeị niili kwịlyeị kwutunatsya. Tinjih tutthug nittṣo tih titi zit tinidhun 


\section{CHUTRUA RSIKOTITINYOO,}

kwikit titettiya, ettinetsi tugwitiyin ukkwa, akọtugwitechya lyẹ, tthui akkwa : kwugguh yoo Vittukoochanchyo ei sso ha kwuntleili ggut tinidhun. 1 Cor. ix.

Ei kwikinjik ha kaönattun ei kaönattun, ukuvanduk tutthug ekoochichyoo nirzi ha. Zyunnunnuwhohnyootäe. rshọ: Vittukoochanchyo yoo elyet chetchosyeị: Kwugguh yoo chitti tte tinjih niili ei yoo kwuttunatsya. Gal. vi.

Ihthle kwidhitilye tinjih tsut nirzị tunotiyin: akọ kwiyendo tsut einut tsut kwikyinjikwizhit kwi zze kkọ zyungịị. Gal. vi.

Vittukoochanchyo vuh nuchud ettet ha ninjiikwudhut ha ukwanji nittsye nilị. Kwugguh yoo chih nunh kug ekoochichyo nukwenyanjik kkwa, ako ettelye kakwutundai shọ ekoochichyo Kethlechidichya. 1 Tim. vi. 6.7 .

Einut chih nunh kug zyungichile akọ tunkohvanyoo, (uhzyunyinjigichöe rshọ akọ kwichile thlih ha tigwinidhun kkwa kwikyinjigoochye rshọ, kọ Vittukoochanchyo sheggu kwundai zit, ei yoo tutthug ekoochichyo kwịlyei ha nuwhoh ntlukwili sso kooli kenjit, kwịrzị kwikit tungitetiya,) koottrid nirzị ha zyunguttaä, chungutterzya, kwu tsuh zyoogguttungittetidichya: kootle chyotlye nirzi enjit tugguthluggutunazya kwi tive kenjit shegg" kwundai zyunkotetun kenjit. $1 \mathrm{Tim}$. vi

Vittukoochanchyo tshitchië vah ukkwa, nuwhoh koottrid, akọ chettigwinidhun ha tigwitiyin kunutande: ei kunkoonyidhohtyin vooh rzi kenjit, rsotitinyookthut kooh tsuh 28 
nohnyoo, akọ thui tte kooh tsuh nohnyoo. Heb. vi. 10.

Kwịrzị tuntohwhoya, akọ kwuntlutohthlya kunnouhnde rshọ: kwugguh yoo tshihtakyi ako tigwinchyo Vittukoochanchyo sso nilị. Heb. xiii. 16.

Chootyin tte chih nunh kug kwu tette tiyin, akọ tuh chah ekoochichyo kih tiyin kkwa nayin, ako yih tsuh trinitsi, nittso tte vih zit Vittukoochanchyo vih chettigwinidhun koọlị ? St. John iii. 17.

Nuwhoh kwutcha kwu ttsun kwuntlouhthli, akọ tinjih nersichachyo ttsye uttsunetunọtaë rshọ: azyuk kwuggut kukwadhud elyet nittsye uttsunetutunethaë. Tobit iv.

Nittso kookwudhun tinitiyin kwikit zyoogguttinidichyo. Kwịlyei kinitiyin chi, lyei unchịli : kwintsul kinitiyin chi, ei kwintsul kwu ttsun sso ha kwuntlunili : akotukoonchyo kwitti dhitsi enjit chunchạzi tuttsun koọlị taị. Tobit iv.

$\mathrm{Ei}$, tinjih nersichachyo akọtounyoo, Kukwadhud heh elnjik: akọ kwinyayin, chitti tte untili, chun kwitti vuh nukwutatsya. Prov. xix.

Ssokolị ei tinjih tinityekthut nersichachyonut ha onassi : Kukwadhud kethle yittutatha kaütseịnukwattshit taị. $P s a$. xii.

\section{T Ako tle Gikhyi akotititenja :}

Tutthug Christ vih ttsyahnut nunh kug zyungịi enjit zyunguttochya.

THLANCHI ha nichinttai ako sheggu Vit1 tuckoochanchyo tinichyo, nih chileh Paul 29 
kirre, kunnuwhohnyidhantyin zyungittitichya, ako zyuntititshik, akọ huị kwuntluntechitaë, tinjih tutthug enjit: chettukotithinyoo ha nih tsut zyungittinichi zyooggutdichi ha chih nuwhoh gichinchi tukwutandul, ei nih ntluntechitaë Kukwadhud Vittukoochanchyo ili : nih tsut zyungittinichi trigichinchilk nikyo zyungịli sheg kooh zit tungwututunaä, thliëtegwitli kwunkyo chihthlug kwiyinji ha, ako thlunkwinadhun ha: Akọ tunkochịnyoo tutthug einut nyooh rzi rsotitinyoo kehzyunkookwanduk, nih kinjik rsotitinyoo kwi thliëtetli zit thlungittinazhya, akọ chihthloog kwiyinji ha zyunkookwutendai, akọ Vittukoochanchyo kit chettigwinidhun ha. Nih tsut zyungittinichi thui kukwadhud njohk thut zyungichinchi, Kukwadhudkthut, hakthuihnut ha tutthug zyunkoohnyạsi akọ kooh kunnyạttyi; ako kwiyendo tsut nih ultsun VICTORIA nuwhoh K wiyeüt : vih kirre Vittukoochanchyo tsut khetachyo ha nuwhoh tsuh keikwutadhud: Akọ tunkohchịnyoo vuh uttunegichikthut tutthug, akọ einut venjit kwuttui kooh nitluntazye, thlih ha ako tsenja tshoogoochoë tigwitiyin akọtungitettiya, troogwandyoh akọ kwịrzi kkwa zyunkwuttunazye, ako nih kwigichinchi thlietegwitli, akọ kwịrzị tigwitiyin kunkutunatya. O Kwittyi zyeh kug, tutthug Gịkhyichoh ako Gịkyinut, kwu tsuh trigwinji koohnittlunchịli koohkwundai ako kooh kunonatun kirre kookunkutunatya nih kinjik thliëtetli akọ kookwundai, ako tshoogoochoë akọ tsenjah tsut nih kuttugwitatshi rsotitinyookthut koohnittlukwutazya. Akọ tutthug nih ttsyahnut zyeh kug kwu ttsut 
nih kwu tsuh trigwinji koohnittluchili; ako kwiyaün tsut chih thluchunachya tzet zyun. gịlị; kwittri huzugitili akọ kwunuchut tsenjah tsut nih kinjik rsotitinyoo zyunkoogootutetthuk akọ zyunkookootendul: thlih ha nyih kunkutanduk rsikotitinyoo ha ako tshoogoochoë tsut tutthug kooh kwundai kwi ttrin zit. Ako chettotithinyoo ha nih tsut zyungittinichi nih chetrsikoonchyo kirre. O Kukwadhud, tutthug einut chih kwundai nyukwudh kwaüttseịnugwichya, zyuntroogoogwitti, kettunzyunkitilị, zyungittinitye, akọ chizi koogoontrhi kwizit zyungịlị. Ako nyooh rzi rsotitinyoo kwu tsut kwịzi zyunginitikhyi nih ultsunnut tutthug enjit chih kwundai kethleh. gichochil nih kwikyinjigwizhit akọ nih nuchut ha: nih tsuh zyungittinichi, kwu tsuh trigwinji nuwhoh ntluchịli kwịrạ kwikit tungettiyin kwikit tuntitudhut, zyeh kug nih kookukwadhud einut ha kuntitiya. Kwikit tochinyoo O Kwittyi, Jesus Christ kirre, ei ri nuwheh chikyidetuggikhyi ako nuwheh Tsbid gịkhyi nilị. Amen.

थ Etchi tai Gikhyi aKotinyoo KuKwadhud ninjikwudhud rsotitinyoo kwuntilutazya, chih kinji akotititenja :

NUWHET tunyinutudhun kendo nukwazi 1 ttrin zit Vittukoochanchyo vih kwu tsuh trigwinji kirre, ako tinihthun einut tutthug rittukoochanchyo tsut ako kwịzi tsut tunginidhun, ei kuttugwitatshi kwintluth hugwizhit Christ vih zyin akọ vuh tta ttsut kooh nittluntihthlya; einut kooh kirre otetundul vih nittitä̈ akọ vuh huinjikwutudhut ginili 31 
kunugandei gelyẹ; ei rzị kwi kirre nuwhoh troogwandyoh ekootichya kunitiyin, ako zyeh kug kookukwadhud kwizit tiya zyunkonitinji. Azyuk kwuggut nuwheh njit rsittuttsut Vittukoochanchyo thlạnchi ha nichinttai nuwhoh Ttyi zyeh kug chettotithinyoo ha ako kwittri ha hui vuh ntluntechitaë, kwugguh yoo vih Tinji Jesus Christ nuwhoh Assi nuwhoh ntlantshi, nuwheh njit ninidhud rzi ukkwa, ko nuwhoh ssi akọ nuwhoh kwundai tthui, ei kuttugwitatshi rsotitinyoo kwizit. Ei yoo Vittukoochanchyo kit akọ hugwizhit koọlị einut tsut ginilị ha zyunkookoonji, akọ nittsọ koukoonyoo einut tsut kunettukotutanja ginilị kkwa ha zyunkookootendul: Seh njit kooli yendo kwedhah nuwhoh tsut ginihkhyi kununyinjitohtah nittso kwichinttsi ei kuttugwitatshi rsikotinyoo, akọ koukoonyoo kwintsi gili ha otetundul: ako kwikit khe nuwhoh uttakwutundai nunnohtye gitshunnohndai, (akọ elyet girsyehtugookwiliị akọ elyet tuchudhiyinkthut kit Vittukoochanchyo ha: ko kwikit tukoonchyo) rsikotitinyoo ha akọ dhitsyạ ei ninjikwudhut zyeh kug kwu ttsun kwu tsuh tohtah, ei nikhut triniditi kwunyikk Vittukoochanchyo kirre tinjikwitizhit uttunututluth rsotitinyoo kwizit zyunnuwhoh tetundul kungịilinut tiyazyunkookoonji ei vih kugguchiäkug rsikotinyoo kwu tsut.

Ei kwu tsut chyo tukoonchyo: Trootshid nuwhoh kwundai akọ nuwhoh nutinde gitshunnohndai Vittukoochanchyo vih tugwitetuggun ha: ako nichin tte uttakunyinjitohyin teko tunowhohyin, kwiyinji ha, kwikinjik ha thui, kookoottrid ha tthui, azyuk khe nuwhoh troo- 
gwandyoh ttrhetohnyoo, ako uttazyunkohndul Vittukoochanchyo thlanchi ha nichinttai tsut, kookwundai etchooggootinjye kinjikootanyoo ha. Ako nuwhoh troogwandyoh kunyinjitohyin Vittukoochanchyo tsut rzị ukkwa, kọ nuwhoh neäg tthui: Azyuk kooh tsuh rsyenunohchi: kwitti zyunkohtsi akọ ette tugwantluth tunwhohyin trichit kookwudhun tunwhohyin kwikit: tutthug koogwichinji akọ teko tigwitiyin chizi tsut tunohwhoyin: ako kwikit zyunohthli kwu tsut oounkootohthli einut kwirzi kkwa nuwhoh tsut tungettiyin, nittso tinjiwhohchi Vittukoochanchyo ttsut nuwhoh troogwandyoh ekooteta: Kwugguh yoo nithlinetsi tigwidichyo ninjikwudhut rsikotitinyoo otinji nuwhoh unkinjiikwitizhit kiyendo tsut kwilye. Azyuk kwuggut ihthle nuwhoh tet Vittukoochanchyo enjit kwịrzị kkwa gịkhyi chi, akọ vih kinjik truttukoochachyo ako kwịrzi kkwa gịkhyi dzetitiyin tthui, kwutsuhkwilhei kugwitrintachik, akọ chizi troogwandyoh kah kooli, nih troogwandyoh kenjit troogwinti, akhekochinyoo, ukkwa chi, ei vihkugchiä rsikotitinyoo kwu tsut nọzyi rshọ; kwurshọ ei kuttugwitatshe rsikotinyoo odhịjik tlẹ, chunkyọ trandyoh nih zit tedha zhit, nittso Judas zit iddhut, ako nih zit troogwandyoh tutthug ha tugwitinili, akọ kwizyin kwunkyo ha rsyehtetginyitathlye.

Ako kwugguh yoo tinjikwitizhit lyee, tinjih ihthle kuttugwitatshe rsikotitinyoo kwu tsuh nutehah lyee, ko Vittukoochanchyo vih zyooggutdichi kwikyinjinizhit rzi, ako uttakwutundai khetachyo ha: azyuk kwuggut nuwhoh tet ihthle koọli chi, vih uttakwutundai dh 
kenjit shọ tih zit tsinettun kwatsi, ko kwiyendo tsut hugwizhit ako uttiigichi tinjitizhit, sih tsuh nuvizi, ko chizi Vittukoochanchyo kinjik kwuultsun vih kwirzi koọli akọ tsenja katundai, akọ nittsọ troogwitti kinikikuttunathlya: Vittukoochanchyo kinjik rsotitinyoo kouinatun kirre vuh tsuh ekootichya otendul, uttiigichi kwunkyo kit otendul, vih uttakwutundai tih zit tsinettun kwittelya, akọ nukweyinjikwitizhit tutthug oounkootazya kenjit.

ब Ko Gikhyi kwunayin chi tinjih Kukwadhud ninjikwudhut rsotitinyoo neginchya kkwa, chih kinjih ei trootshid kwitti akọtititenja :

KWUCHANUT kwintluth nuwhet tunyi1 nutudhun, nukwazi ttrin zit kendo akotinihthun, Vittukoochanchyo vih chetrsikoocnhyo kirre, Kukwadhud khah tsut chutruä kunukwandai ha kookutunelhtya : ei kwu tsut Vittukoochanchyo vooh rzi ha, nuwhoh tutthug tzet zyunohthị nutohta akọtunnuwhakhnyoo: ako tunnuwhoh chihnyoo, $\mathrm{Ku}$ kwadhud Jesus Christ venjit elyet unkwuntutudhoha ei kwu tsuh nutohta, chettigwinidhun ha nuwhọkkunginchi Vittukoochanchyo attun thun kirre. Kazyunwhuhndai nittso kwittsitukoonchyo ako rsinichi kkwa, ninjikwudhut kwichile tinjih rsyenugwiei taị, tih vuhkugchiä ssi uttutonyachyo nouitinethli, azyuk kwuggut ekoochichyo kookkuttigwinidhun kkwa einut vookuginchi, guttutatsha tsut kwirzị akọ kwutturug einut vookunginchi(kwugguh kkwa) kwintluth hui kwinidhun kkwa elyet kweẹ tunginidhun. Chootyin rsit nuwhoh 34 
tet kwikit tukoonchyo koọli elyet ninjitulhndai? Chootyin tteakotiyitineshya kwintluth tshoogoochoë kkwa akọ tekọ akọtayin? Azyuk kwuggut, $\mathrm{k}$ wintluth nuwhet tunyinutudhun Christ zit, koochid rshọ, chih khah tsut chutrua rsikotitinyoo kethle tuttutohzi chi, Vittukoochanchyo vecho uttuttsut zyunnyidhotzye. Kiyikwudhun koọli tinjih akọtititenja elyet otudhihndul, kwugguh yoo kisigwichaë nunh kug kwi echichi kirre. Ko akọtukoonchyo tugwinyoo elyet sso ha kotinji ako akheötitinji Vittukoochanchyo ndah. Tinjih ihthle akotinyoo chi, treshit trelhndyoh azyuk kwuggut kwunulhchut nutihha; chyaggut tte thlitseyinjitinidhohzhit ako rsyenudhohzhit? Vittukoochanchyo nuwhọh kungikhyi taị, lyet truttunnohnji akotuntohnyoo elyet kweetudhota? Etchi taị Vittukoochanchyo vuh tsut uttsunetutunohta lyẹe, lyet uhzyunguttohchya, akọtuntutohnja elyet rsyehnudhohchya? Tsenja tsut ettininjiohchi tugwantsul tsut akotukoonchyo tunchoui ha tuntohnyoo Vittukoochanchyo ndah khunkoogwitazya. Sso kooli Kwunduk zit einut ninjikwudhut elyet kwu tsuh tunginizhin, kwugguh yoo kwunzi tekit zyunkookovịkthut, ko kuttu aki kunkookoonjik, kọ nikhut triniditi gelyẹ, akotukoonchyo kwenjit ekoochiyo elyet tunkotithinyoo: ko elyet kungilya zyeh kug ninjikwudhut kenjit akotunkotetinyoo. Sih yoo rsyenutihsya ; akọ suh kuttukootatshe kit akọtunnuwhohchihnyoo Vittukoochanchyo vooh rzi ha, nuwhọh kunginihkhyi Christ enjit, nuwhoh tsuh ginihkhyi, nuwhun thun nuwhoh chassi ket-tunyinoohthun kit, chih thlunovatun 
rsikotitinyoo nikha zyunotohndul. Akọ nittsọ Vittukoochanchyo Tinji akọtinizhin tuchun kwinititaë kug ninidhud, tuh nkyọ kwuntlantshị nuwhoh chassi kenjit: kwikit tukoonchyo nuwheh njit koogwichiltshei koọli thlunovatun zyunotohndul kunnutondai kenjit ninidhud kwi utteinjikwatsi, nittso thun kuttukoochildhut: ei akọtunwhohyin kkwa chi, ettininjiöhchi, tugwantluth Vittukoochanchyo tsut kwịrzi kkwa tunwhohyin, akọ nittsọ kwursanyië kookwunazye nuwhoh tshih tig nuntutsei; kinjikootanyoo ha Kukwadhud vihkugchiä ttsun kwudhohnji, akọ nuwhoh chakthut ttsut dhohchyah, einut zyeh kug kwi ttsi kwi ninjikwudhut kwu tsuh niginchya zyunuguteä kenjit. Chih ekoochichyo tsenja tsut ninjiohchi chi Vittukoochanchyo vih kwu tsuh trigwinji ha, kwiyinji kwiyendo tsut nirzi, kwu tsuh uttsunetutunohta ; ei kuntohwhoya kenjit elyet akhezyunkotitidhitinja, Vittukoochanchyo thlanchi ha nichinttai nuwhoh Ttyi zyeh kug vuh tsuh zyunguttutichya.

ब Ninjikwudhud rsotitinyoo Kwuntlekwanji tai, Gihki chih kinji akotititenja.

TUWHET tunyinutudhun Kukwadhud 1 zit, nuwhun nuwhoh Assi Christ vih kuttugwitatshi rsikotitinyoo, kwu tsuh titutah zyunyinoohthun, kununyinjitohta lyẹ nittsọ Paul rsotitinyoo tinjih tutthug tsuh gikhyi tsenja tsut ettitshungutandai, kunettukotanyoo kwiyetshi ei Thlyedh zyunguä, akọ ei vih zit ettritinyin zyunegitinyin. Kwugguh yoo nittsọ uttrigwittäei kwichintsi nili, kwittri 36 
thlih ha thlitseyinjikwitunzhit ha ako kwikyinjigwizhit kwundai ha ei kuttugwitatshi rsikotitinyoo zyunkonitinji : (kwugguh yoo akọ taị Christ tthuị kwunkyọ kit zyunituä, akọ vuh tta zyunitinyin; akọtai Christ zit tuniảichyo, akọ Christ nuwhoh zit: Christ ha chihthloog zyunitili, ako Christ nuwhah nili ;) kwikit tukoonchyo koukoonyoo kwichintsi, ginili kkwa ha zyunkonitinji chi. Kwugguh yoo akọ tạ nuwhoh Assi Christ zyin akọ vuh tta kwu tsuh kwịrzị kkwa tunitiyin; nuwhun thun nuwhoh unkinjiikwitizhit zyunituä ako zyunitinyin, Kukwadhud zyin kununyinjiidhadhut; Vittukoochanchyo vuh vecho uttutsut zyuntakyi uttutsut zyunnyidhatsye nuwhoh teggah tsik nithlinetsi tunthichyo ha, ako kwitshị nithlinetsi tunthichyo ha. Azyuk kwuggut kwuchanut uttunyinjotohttre Kukwadhud ttsut elyet nuwhoh telhttre: nuwhoh troogwandyoh kenjit thlih ha zyuntroogoohti: Christ nuwhoh Assi kwikyinjigwizhit kookwundai ako yinjikwichintluth ha vih kyinjohchi; nuwhoh kwundai thlitseyinjitunohthli, ako tinjih tutthug ha thlih ha nikhut tunyinohwhudhun; akọtukoonchyo ei nunaị rsikotitinyoo tsenja tiyazyunkookonji zyuntohthlya. Ako tutthug ekoochichyo kwiyendo tsut Vittukoochanchyo tsut Kwittyi, Kwitinji, akọ Chunkyọ Rsotitinyoo hui kwuntluntututohthlya, nunh kwitizi kenjit nuwhoh Assi Christ ninidhud akọ huịjiithudhut kirre, ei yoo Vittukoochanchyo nili ha; ei uhzuggutidichyo, kwinititaë kug ninidhud, nuwheh njit zyuntrandyoh huịnjiidichi, nuwhun tsegga kwizit akọ tshitshi 
kwunkyo kwizit zyunitilye; Vittukoochanchyo kkyinut zyunnuwhoh tatsyah, akọ sheggu kwundai $\mathrm{kwu}$ tsut nunnuwhoh tathlye. Akọ nuwheh Kukwadhud, akọ ei rzị nuwhoh Assi Christ vih chettigwinidhun treshit kwichintsi sheggu kunutandai kenjit, nuwheh njit kwikit ninidhud, akọ kwi echichi nirzị shọ kwittitiditshi, ei vuh tta kwichile nuwhenjit gitunja, nuwheh njit uhgwittäeị; kuttugwichiltsheị, akọ kuttukoochildhut nugwinaị rsotitinyoo, vih chettigwinidhun tsut koogwiteyin nilị. akọ ninidhud sheggu kunutandai kenjit, nuwhoh uhgwizhit kwichintsi, akọ kwilikwutetha. Azyuk kwuggut vuh tsut, $\mathbf{K}$ wittyi, Chunkyo Rsotitinyoo ha, (nittso nuwheh njit tinjikwitizhit) huị sheggu kwuntluntechitaë; ettikwuntlutichazi thlanchi ha vih yinji akọ kettigwinidhun kwu tsut, akọ koọkkunnitityi thlih ha rsikotitinyoo akọ tshoogoochoë tigwitiyin ha vih kuntutanduk nuwhoh kwundai kwi ttrin zit tutthug. Amen.

Tा Ako tle einut tsut ninjikwudhut rsotitinyoo kwu tsuh neginchya zyunkookotendul kenjit, Gikhyi akotititenja:

NUWHUN thlih ha ako kwittri ha nu1 whoh troogwandyoh kenjit zyuntroogoohti, akọ nuwhoh neäg tutthug enjit nuwhoh chettigwinidhun, ako nuwhoh zyooggutdichi koọli, ako kechid kookwundai tuntitudhut zyunyinoohthun, Vittukoochanchyo vih tugwitetuggun kwikit tohchya, ako chooggoo kwu ttsut vih nutinde rsotitinyoo kwikit kunwhohtul: Kwikyinjigwizhit ha kwu tsuh nunohchya, akọ chih kutturwitatshi rsiko38 
titinyoo zyunonohnji nuwhoh uhgwizhit kwu tsut; ako uhzuggutigwidichyo Vittukoochanchyo tsut uttazyunkohnduk ettritsoogoochichyo ha nuntshikwutchidhohe.

Tा Ako tle chih ket kwanduk alkọtititetinja Gikhyi kirre: attun tutthug tinjih ha muntshikwuttrule.

ITTUKOOCHANCHYO thlanchi ha
nichinttai tinichyo, Jesus Christ nuwheh Kukwadhud vih Ttyi, ekoochichyo kwuttutthug kwudhantsei, tinjih tutthug Untoutitinyanttri: Nuwhoh troogwandyoh kwịlyeị ako nuwhoh koottsite kazyunkwanduk ako kuttrhechanyoo, ei yoo ndonithlig treshit tsut zyunkwudhantsei, Kwiyinji ha, kwikinjik ha, ako kookoottrid ha, Vittukoochanchyo Kukwạdhud tsut, thlih ha nih vecho ako nih zinatsye nuwhoh tsut uttu ttsun unchitaë. Thlih ha thliseyinjitinidichi akọ kwittri ha zyuntroogwititi chih nuwhoh troogwandyoh kenjit; kunnandai taị zyuntroogwititi; kwu kdha kookoonttrhi. Nersingichachyo tunnuwhoh chinyoo; Nersingichachyo tunnuwhohchịnyoo, Kwittyi kwintluth nyeh rsikoonchyo: Jesus Christ nih Tinji, nuwheh Kukwadhud vih kirre tutthug kwuttokwuchodhut nuwheh ooünkoochịli : Ako tochịnyoo chooggoo kwu ttsun sheggu nih kuntutanduk ako sso nih tsun zyuntatsya kechid kookwundai ha. Nyooh rzi kwiyinjikwichile akọ kwi nindelh ; Jesus Christ nuwheh Kukwadhud kirre. Amen.

\section{Ako tle Gikhyi (kọ Gikhyi choh dhiti chi) 39}


nutedhut, ako chih troogwandyoh ekootichya tinjih tsut akotititenja:

IITUKOOCHANCHYO thlanchi ha nichinttai, Nuwhoh Ttyi zyeh kug, ei vih zyooggutdichi chintsi kirre einut tutthug thlitseyinjikwitunzhit kwittri ha ako thlih ha kwikyinjigwizhit ha gih tsututtsunetuntakooh ttsut troogwandyoh elkootichya khenitteitinitäeị: Nersingichachyo tunnuwhoh choonyoo ; nuwhoh troogwandyoh nuwhet oounkoochoola, akọ ei kwu ttsut kethleh nuwhoh choolya, nuwhoh yunvinji ; tutthug kwịrzi zit nunnuwhoh choosyei ako nunnuwhoh choottai ; sheggu kwundai tsut nunnuwhohlya; nuwheh Kukwadhud Jesus Christ kirre. Amen.

\section{बा Ako tle Gikhyi akotititenja :}

Zyunkodhudhohtshẹ ei kinjih uhgwizhit nuwhoh Assi Christ einut gih tsuh uttsunnutunta, akọtunnanyoo:

@EH tsuh nunohchya nuwhoh tutthug $\checkmark$ koottridtungohyin oko nuwhoh kwudhah tugwinti, ako nukwazi nuwhoh ntlukootutihthlya. St. Matt. xi. 28.

Vittukoochanchyo nunh kwikit kettinizhin tih Tinji chihtloog rzi kwuntlantshị, ei kwu tsut chootyin tte yih kyinjizhit rsyetetgittitethlye, kọ sheggu kwundai tetiya. St. John iii. 16.

Chitti Paul rsotitinyoo nyoo tthui zyunkcdhudhohtsheị :

Chih yoo kinjih thliëtegwitli, ako gitetchịi tinjih tutthug zyunkookotendul : ei yoo Christ 40 
Jesus nunh kug nuịzi trandyohnut tassi kenjit. 1 Tim. i. 15.

Chitti John rsotitinyoo nyoo thui zyunkodhudhohtshei :

Tinjih ihthle troogwandyoh titiyin chi, Kwittyi ha Tshidgịkhi vunitiyin, Jesus Christ tshitchoë ; akọ nuwhoh troogwandyoh keh njit ttsinettun kwatsi nilị. 1 John ii. 1.

\section{बा Ako tle Gikkhi akotititenja.}

Nuwhoh ttri nuttohthlih.

Kwitti kwigikhyi. Kukwadhud tsut nittitili.

Gikhyi. Nuwheh Kukwadhud Vittukoocharchyo huị vuh ntlutotilya.

Kwittikwigikhyi. Etteye rsittuttsut ako tshoogoochoë kwikit tugwitechya.

ब Akọ tai Gikhyi Kukwadhud vihkugchiä tsut nudhut, akotititenja.

D SITTUTTSUT, tshoogoochoë, akọ nuwheh $\mathrm{n}$ njit tinjikwitizhit koọli. sheggu tsut, akọ tutthug kwitekit hui nyih ntluntutitaë lyẹë O Kukwạdhud, *Kwittyi Rsotitinyoo, Thlanchi ha nichinttai tinichyo, sheggu Vittukoochanchyo.

[*Chih kinjih (Kwittyi Rsotitinyoo) elyet akotititethinja Trinity nukwazi ttrin zit tai.]

I Tzet tshoogoochoë kwiyetshi kwikinjik, akotukoonchyo kooli kuttugwitatshi akotititetinja; ko chih inchit tsut akotititetinja: 
CHUTRUA RSIKOTITINYOO,

ZYUK kwuggut zyehket-kutchin, akọ A zyehket-kutchin tshidkthut ha, akọ tutthug zyeh kug nihazyunkitili ha, nyooh rzi kwichinttsi zyuntettitchidhaë, akọ zyuntetchatsi : sheggu zyuntenyititchidhaë, akọtunchitinyoo, Rsochịtinyoo, rsochịtinyoo, rsochịtinyoo, Kukwạdhud lyeịnut kooh Vittukoochanchyo ịlị, zyeh kug nunh kug ha nih chinttsi ha tunkootinilei : Kwichinttsi nih tsut koli, O Kukwạdhud kwiyendo tsut kwuttug tinichyo. Amen.

\section{Tshoogoochöe Kwiyetshi Kwikinjik.}

Christ vuh kooli ttrin zit, ako chitsuëtsinekthui ttrin zit tle.

WUGGUH yoo Jesus Christ ette vih rzi I. nih Tinji kwuntlantshị chooggoo kit tukoonchyo nuwheh njit vih kwịlịh: ei Chunkyo Rsotitinyoo kirre thlih ha tinjih ulttsei tuh hun nichid Mary ttsut : akọ troogwandyoh koogootettukthut kkwa, troogwandyoh kwu ttsut nuwhun uttata. Azyuk kwuggut zyehket-kutchin ha, \&c.

Ninukwalye Ttrin zit, ako chitsuëtsinekthui itrin zit tle.

To kwiyendo tsut nuwheh njit tinjikwitiI zhit zyuntehnyihtettaä nih Tinji Jesus Christ nuwheh Kukwadhud vih nunukwalye chinttsi; kwugguh yoo thlih ha tivi kki kekokoshit nili, nuwheh njit kwuntlantshị, ako nunh kug kwi troogwandyoh oounokonjik; 
ei ninidhud kirre etshị rsyetetgichiltseị, akọ zinelikhyin kirre sheggu kwundai nuwheh njit khungwithäeị. Azyuk kwuggut zyehketkutchin ha, \&c.

Gittikwichizhit Ttrin zit, ako chitsuëtsinekthui ttrin zit tle.

NIH Tinji kwintluth vet tinịhun Jesus 1 Christ nuwheh Kukwadhud kirre; ei kwchinttsi ha ninelikhyin tle kwirzye tsut vih kwitinchin tutthug vih chilhe tsut, ako kooh ndah zyeh zyọ nethizi nuwheh njit kwitekit rsyenukooteä; nichin tte tinchyo, azyuk kwu tsuh guttutituta, ako vah kunkwutadhud nindelh zit. Azyuk kwuggut zyehketkutchin ha, \&c.

Takui Nukwazi Ttrin zit, ako chitsuëtsinekthui ttrin zit tle.

JESUS Christ nuwheh Kukwadhud kirre: ei vih kheniteikwichitäei kwintluth thliëtetli kit, Chunkyo Rsotitinyco nuteidhut zych kug kwu ttsun chooggoo kit tinchyo, kwintluth kwuttudhatshik inchit tsut ha, kwintluth attrui kit tinchyo kkontsha kit tinchyo, chilhenut tshih tig nukko nunchodhut, kunkotunachya keh njit, akọ thliëtetli tutthug $\mathrm{kwu}$ tsut zyunkoohtuneä; nithlinetsi kwikinjik ozi kooh nittlunchotli, akọ yinjikwichintluth kinjikootanyoo kwichokkun ha sheggu tsut tutthug kutchin tsut ssokoọli koo Kwunduk zyunkookootanduk; ei kwi kirre tsegga ako kwinetsi tigwitiyin kwu ttsun nunnuwhah zye attri kwechin kwu tsut, ako thlih ha nyah 43 
CHUTRUA RSIKOTITINYOO,

zyunitundai, akọ nih Tinji Jesus Christ. Azyuk kwuggut zyehket-kutchin ha, \&c.

\section{Trinity ninjikwudhud tai rzi.}

NUN chihthloog Vittukoochanchyo ịli, 1 chihthloog Kukwadhud; elyet chihthloog rzi Kwundai, kọ ttyid Kwundai chihthlud vih zit. Kwugguh yoo ei kwikyinjidichi kwittyi nindelh kenjit ei chun kwitinji enjit kwikyinjidichi ako Chunkyọ Rsotitinyoo enjit, nithlinetsi tunidichyo, ako thlunachyo. Azyuk kwuggut zyehket-kutchin ha, \&c.

वा Ei kwiyetshi kwikinjik tutthug tle inchit tsut chih kinjih akotititenja :

A ZYUK kwuggut zyehket-kutchin, ako A zyehket-kutchin tshidkthut ha, akọ tutthug zyeh kug nihazyunkitili ha, nyooh rzi kwichinttsi zyuntettitchidhaë akọ zyuntetchatsi : sheggu zyuntenyihtitchidhaë, akọtunchitinyoo, Rsochịtinyoo, rsochịtinyoo, rsochịtinyoo, Kukwạdhud lyeịnut kooh Vittukoochanchyo ịi, zyeh kug, nunh kug ha nyih chinttsi ha tunkootinilei : Kwichinttsi nih tsut kolị, O Kukwạdhud kwiyendo tsut kwuttag tinichyo. Amen.

ब Ako tle Gikhyi Kukwadhud vihkugchiö tsut nitshikwutä, tutthug einut ninjikwudhut rsotitinyoo zyunkookotendul enjit chih kwigichinchi akotititenja :

HLYET kunetotudhanyoo chih nih vuhkugIf chiä kwu tsut ninidichya, O Kukwạdhud nyeh rsikoonchyo, nuwhun thun nuwhoh 44 
tshoogoochoë tigwitiyin kunkovitili, kọ nih zyooggutdichi kwịlyei, ako kwichintsi kirre. Elyet gunitili ei yoo nyih vuhkugchiä zuggu chooliddi zyunnyatsi. Ko nun ihthloognchi Kukwadhud ịlị, sheggu zyooggutdichi nitiyin : Azyuk kwuggut, Kukwadhud nyeh rsikoonchyo, akọtunnuwhoh chịnyoo, nih Tinji Jesus Christ vettinịdhun vih zyin kwikit zyuntituä, ako vuh tta zyuntitinja, nuwhoh zyin trandyoh vih zyin kirre rsyunkwutanja, ako nuwheh nkyo vuh tta kwintluth kwichile kirre kunnutatrha, ako sheggu vih zit tuntidichya, ako attun nuwhoh zit. Amen.

T Gikhyi vihkugchiä kwundah nudhut tsenjah tsut thlyethchyo chugchoo ha niniei tai, chith kwigichinchi rsotitinyoo kookwatsi akotititenja :

VITTUKOOCHANCHYO thlanchi ha nichinttai tinichyo, nuwhoh Ttyi zyeh kug, nih zyooggutdichi chinttsi kirre chihthloog rzi nih Tinji Jesus Christ kwuntlantshị tuchun kwinititäe kug ninidhud nuwhoh chassi kenjit : ei yoo azyuk kweltseị (atteịnjiettichiltsei, chihthlud kwuntlatshị) thloogoodichyo, thlanchi ha ettetugwantsi atteinjikwatsi, ako sso kwatsi, tutthug nunh kug koo troogwardyoh kenjit; akọ kuttukoochiltshei, akọ vih kwunduk nirzi rsotitinyoo ha nuwhoh tsuh kuttukoochildhud zyuntititindyah, sheggu kunukwandai, ei vih tshi chile kunutetik kwu tsut: Nuwhoh dhudhantshei, O Kwittyi zyoogguttinidichyo, kwintluth chettotithinyoo ha nih tsut zyungittinichi; ako tochịnyoo chih dhantseị thlyethchyo chugchoo ha, 
tukwanji, nih Tinji Jesus Christ nuwhoh Assi vih kuttukootatshi rsotitinyoo kit, ninidhud akọ huịnjithudhut kunukwandai gelyẹ, vih zyin ako vuh tta kwintluth chile kunnotandul: ei yoo ei ttodh nuane ha kwuntlatshị thlyethchyo oonjik ; ako huị kwuntlutịeị tiẹ, thleyozudh, akọ tuh kaönattunnut nuttlunyotli, akọtitịnyoo ; Zyunonohnji, zyunoha, chih yoo sih zyin nuwheh njit kwuntlatshi : Chih kwikit tunwhohyin suh nutondai kenjit. Ako khah tsut chutrud tlẹ,' vih zit ettritinyin oonjik: akọ huị kwuntlutịi tlẹ, kooh nittlunyokuị, akọtitịnyoo: Nuwhoh tutthug chih zyunwhohnyin; kwugguh yoo kechid thlugwinadhun ttsut chih suh tta nili, nuwheh njit ako lyei enjit gitunja troogwandyoh ekooteta kenjit: Chih kwikit tunwhohyin tugwinyachiddi zy unuttowhuhnja, suh nutondai kenjit. Amen.

I Ako tle Gikhyi trootshid ehthletsut, thlyethchyo ako chugchoo kotendul, ako kootle Gikhyi choh, gikhyi ha, (zyungili chi) kooh nittluntelya, ako tle tinji thui tutthug khetachyo ha nuntshikwuttrule. Ako thlyethchyo ihthle kwuntlunili tai akotititenja:

WUWHEH Kukwadhud Jesus Christ vih I zyin nyeh njit kwuntlatshị nih zyin ako nyuh nykọ sheggu kwundai kwu tsut kookunyooti. Chih ooninji, nyuä, kununyandai Christ nyeh njit ninidhud, ako nih ttri zit vuh kkọ chịa kwikyinjigwizhit kirre huị ha. 
बI Ako gikhyi vih zit ettritinyin ihthle tsut kwuntlutäei akotititenja :

TUWHEH Kukwadhud Jesus Christ vuh 1 tta, nyeh njit gitunja, nih zyin ako nyuh nkyo sheggu kwundai kwu tsut kwikunyooti. Chih nyitinyin, kununyandai Christ vuh tta nyeh njit gitunja, akọ huị yinọzhin.

๙ Tutthug zyunkookoonjik tle. Gikhyi Kukwadhud vihkugchia tsut tehah, ako $K u$ kwadhud gichinchi alsotititenja, tinjih tutthug gihkit-teingittekhya.

WWHOH Ttyi zyeh zit nih kwilnjik. 1 Nyooh rzi chootsi. Nih kookukwadhud nuvizi. Tinịdhun nunh kug tugoochyo, nittso zyeh zit troogwatsi kwikit. Chih ttrin zit nuwhun enyantsit ttrin ndo thloogootunazya nuwhoh ssih. Nuwhoh troogwandyoh nuwheh unkoochịli nittsọ kooh ttsut unkoochitili einut troogwandyoh nuwheh tungittiyin. Kookukwundai kwu tsut nuwhoh tunọë rshọ; kọ troogwandyoh. kwu ttsut nuwhoh yunninji. Kwugguh yoo nih tsut nili kookukwadhud, ttai, akọ nindelh sheggu kenjit akọ sheggu kenjit. Amen.

9ा Ako tle akotititetinja :

KUKWẠDHUD ako Kwittyi zyeh kug nuwhun nih ultsunnut uhzuggutunidichyo thlanchi ha kookuttunyinitudhun kwittyi kit nih zyooggutdichi chetrsikoonchyo ha nuwhoh utteịji kwatsi, kwittugootichile akọ huị ha oninji; kwintluth chettukotithinyoo ha nih tsut zyungittinichi akọ tochịnyoo, nih Tinji Jesus Christ nittso ginilị, akọ vih tshi kirre, akụ vuh 
tta zit kwikyinjigwizhit kirre, nuwhun akọ tutthug nih ttsyahnut nuwhoh troogwandyoh ekootichya kuntitiya, ako tutthug chizi ubkookwazi huinjithudhut kirre. Akọ tzet at. teịnjizyunatsi, akọ nyih ntlunatshi. O Kukwạdhud, nuwhun thun nuwheh nkyọ nuwhoh zyin ha, kakwitundai ha, rsikotitinyoo, akọ kwundai atteịnjikwatsi nih tsut kwittelya : chettukotithinyoo ha nih tsut zyungittinichi nuwhoh tutthug chih kuttukootatshi rsikotitinyoo zyunonitinji, nih chetrsikoonchyo ako zyeh kug nih kwịrzi tigwitiyin ha nuwhoh zit tungwitutunaä. Akọ kwuttuzug elyet gunitili, nuwhoh troogwandyoh kwillyei kirre, ekoochichyo atteinjikwatsi nyih ntluntititilya; Ko nih tsut zyungittinichi chih ettelye kwikit tuntitiya, akọ nuwhoh koottrid nili oninji : nuwhoh ttsun kookoọlị Keịkwitọtye rshọ kọ nuwhot troogwandyoh oounkootọlya Jesus Christ nuwheh Kukwadhud kirre; vih kirre akọ ei ha thlukoovatun zit Chunkyọ Rsotitinyoo ha, tutthug yinjikwichile ako nindelh nih tsut Kolị. O Kwttyi thlanchi ha nichinttai, kwilitkwutetha kwu tsut. Amen.

\section{Ko chih.}

THLANCHI ha nichinttai ako sheggu kwu1 ndai Vittukoochanchyo tinichyo. Kwintluth tsut kwittri ha huị nyih ntlunchitaë, Kwugguh yoo nuwhoh ạa ssoịli nuwhun chih nunaị rsikotitinyoo tsenja zyunodhitinjik, truä kwunkyo kit ha nih Tinji, nuwhoh Assi Jesus Christ vih zyin ako vuh tta kwintluth tatluth; akọ ei kwi kirre nuwheh kikyunandai nih chetrsikoonchyo akọ nih zyooggutdichi nuwhoh 48 
tsut; ako thlih ha kookwiyerzra kwittsi zyunkwittititshi zyunitili nih Tinji zyin nunai, ei yooehthlugoo tigwidichyo ssozyunkwatsi tutthug tinjih kwikyinjigichye; ako kuntrookolinut zyungili thui kukonyoo kwikirre ei nih kookukwadhud sheggu kooli, nih Tinji vettinutudhun ninidhud ako huịnjithudhut kwintluth tatluth kwiginili kirre. Akọ kwintluth chettukotithinyoo ha nih tsut zyungittinichi. O Kwittyi zyeh kug, $\mathrm{K}$ wikit nuwhoh tsuh trunyundai nih kwu tsuh trigwinji ha, ei kwichya rskotitinyoo zit zyuntititindya, ako tutthug ei koottrid nirzi chitti tte nuwheh nyit rsyenukooniei kwikit titutah; Jesus Christ nuwheh Kükwadhud kirre, ei tsut nun ha akọ Chunkyọ Rsotitinyoo ha, tuthug yinjikwichile akọ nindelh kolị nunh kwilikwutetha. Amen.

\section{Akọ tle akotititetinja, ko ettitetilya:}

ITTUKOOCHANCHYO tsut nindelh
koli tukkudhud, ako nunh kug ttsinettun, kwịrzi tigwinidhun tinjih tsut. Tunnyitichidhaë, tunnyitatsye, nih tsut zyungittinichi, zyunyinjinihchidhaë, huị nyih ntluntechitaë nih niudelh kwichinttsi gelyẹ. O Kukwạdhud Vittukoochanchyo tinichyo, zyeh kug Kukwadhud njo, Vittukoochanchyo Kwittyi Thlanchi ha nichinttai tinichyo.

O Kukwạdhud, chihthloog rzị Kwitinji Jesus Christ, O Kukwạdhud Vittukoochanchyo, Vittukoochanchyo ttsut tivikkyi, Kwittyi Tinji, nunh kug kootroogwandyoh oountukwạjji, nersingichachyo tunnuwhoh chịnyoo. Nun nunh kug kou troogwandyoh oountu49 
kwanjị, nersingichachyo tunnuwhoh chịnyoo. Nun nunhkug kootroogwandyoh oounkoochili, nuwhoh gichinchi oninji. Nun Vitukoochanchyo Kwittyi nandei tsei dhinti, nersingichachyo tunnuwhoh chịnyoo.

Kwugguh yoo nun rzị rsochitinyoo; nun rzị Kukwądhud ịlị; nun rzị O Christ, Chunkyo Rsotitinyoo ha. Kwiyendo tsut kwiyettug ili Vittukoochanchyo Kwittyi vih nindelh zit. Amen.

- Ako tle Gikhyi (ko Gikhyi choh dhiti chi) chih kwigichinchi ha tinjih kethle gitetah otitenja.

VITTUKOOCHANCHYO vih ttsinettun, ei tutthug kwikittutzigwele yendo tsut kwichoë, nuwhoh ttri, nuwhoh yinji ha kookunyooti. Vittukoochanchyo vih kakwutundai, ako vih chettigwinidhun zit ako vih Tinji Jesus Christ nuwheh Kukwadhud ttsun; akọ Vittukoochanchyo, Kwittyi, Kwitinji akọ Chunkyo Rsotitinyoo, vih zyooggutdichi, nuwhah kolị, akọ sheggu nuwhah chooë, Amen.

I Kwigichinchi tititetinja atteinjikwatsi tle, Kuttukootatshi kooli kkwa tai, akotukoonchyo ttrin chihthlud ko kwiyendo tsut; ako ei kookwudhun tuntitetinja tthui tugwinyachiddi tinjikwitizhit kooli vunkwigichinchi Ko Khah tsut kwigichinchi tle, Kuttukootatshi ko Litany tthui gikhyi kwirzi tinidhun kwikit.

KUKWADHUD, chetrsikoonchyo ha nuwhoh tsuh trunyundai, chih nuwhoh 50 
kwittitshik, ako nuwhoh kwigichinchi ha akọ nih ultsunnut kooh $\mathrm{kwinië} \mathrm{rsitkwịli} \mathrm{she}$ ggu tsut kwi chassi kwitetiya kwu tsut; tutthug nikhetungwichithäe ako kwunzikootazhi kwittet chih kwitshị kwundai nih kwutsuh-trigwinji rsinichi akọ inchit tsut koọị kirre sheggu kookunkwuttunatya; Jesue Christ nuwheh Kukwadhud kirre. Amen.

THLANCHI ha nichinttai Kukwạdhud, akọ sheggu Vittukoochanchyo, tinichyo, akotinọzhin nih tsut zyungittinichi, ndokwuttanta, rsikotitinyoo kotsya, akọ kwu tsuh Kukwadhud nuwhoh ttri nuwhoh zyin ha, nih tugwitetuggun koo kwinië zit, akọ nih kuttukootadhud kwi koottrid zit; nih kookunatye nukoochinttai kirre tze tako sheggu, kwizyin zit ako kwunkyo zit, rsyeh nuwhoh kukootunatya ; nuwheh Kukwadhud akọ nuwhoh Assi Jesus Christ kirre. Amen.

KOTINOZHIN. Thlanchi ha nichinttai A vittukoochanchyo tinichyo, nih tsu, zyungittinichi, ei kinjin nouggoo ttrin zyuntitutthuk nuwhoh tzi kwuudh ha, nih kwutsuhtrigwinji ha nuwhoh ttri zit nuntontthui, nuwhoh zit kwịrzị kookwundai kwuttatsyanyooh rzi kwi yinjikwichile akọ tugwitichile tsut; Jesus Christ nuwheh Kukwadhud kirre. Amen.

KUKWADHUD nuwhoh yetshih chịheih, tutthug nuwhoh kottrid zit nih kwutsuh-trigwinji rsinichi ha, ako nuwhoh tsuh trunyundai nih kwutsuh-trigwinji sheggu tsut ha; nuwhoh koottrid tutthug nyah kukakwinyoo shid, kwittichootinyin, ako kwili-

51

D 2 
keozhid, nyooh rzi rsotitinyoo zyuntututaä keh njit, ako huịko tsut nih zyooggutdichi kirre sheggu kwundai kuntitiya ; Jesus Christ nuwheh Kukwadhud kirre. Amen.

THLANCHI ha nichinttai Vittukoochan1 chyo tinichyo tutthug kwirzye hundhutluị, chitti tte nuwhoh tinjikwitizhit kanyitundai zyunochitukthut kwiyetshi, akọ nuwhol katrigwitinjik zyunochitukthut tai ; nuwhoh nutrigwichintlok keh njit nersingichachyo tunnuwhoh chịnyoo; akọ ei ekoochichyo nuwhoh gili keh njit shọ kunettochanyoo, akọ nuwhoh nde-ettun keh njit shọ zyunochitukthut, nuwhoh ntlunili akọtinọzhin, nih Tinji Jesus Christ nuwheh Kukwadhud vih ginili kirre. Amen.

ITHLANCHI ha nichinttai Vittukoochan1 chyo tinichyo nun khenitteitinịtäei otudhantsha kooh kwigichinchi einut nih Tinji vooh rzi kirre zyunkootutukthut: Nih tsut zyungittinichi rsinichi ha zyunnuwhoh dhudhạntshei, nuwhun chooggoo nih tsut zyunititshih, akọ nih tsut zyungittinichi ; akọ tinozhin, ei ekoochichyo, kwikyinjigwizhit ha zyunotitukthut nih tigwinidhun kit, thlih ha kuntitiya, nuwhoh tinjikwitizhit tugwitutunaä, akọ nih nindelh kukootunatya tsut; Jesus Christ nuwheh Kukwadhud kirre. Amen.

I Nulkwazi ttrin zit, chizi ttrin zit tthui (Kukwadhud khah tsut chutruä rsotitinyoo kwuntlukwittäei kkwa chi) tutthug kwigichinchi kooli ninjikwudhud rsikotitinyoo keh njit, ei kwu tsut ei kwigichinchi tutthug Christ vih ttsyahnut nunh kug zyungili enjit akotititetinja. 
" TV WUGGUH yoo chih kuttukookwichiltsei kwizit, ei yoo Kukwadhud khah " tsut chutruä rsikotitinyoo nittso kwuntlu" kwittäei keh njit kwikit kuttukootadhud, " einut zyunkookoonjik nuntshikwuttrule " zyunkookotendul; ei kuttukookwichiltsei " kwịrzi tsut kwinidhun, ei yoo akọtigwini" dhun tigwinidhun, nittsọ chettukotithinyoo " ha akọ huị ha zyunkotinji Christ vih ozi " nirzị; ; ei kwikirre tutthug einut zyunkigịli " zyunkookoonjik kooh ttsun zyunkookwattsi, " ako kwịrzi tsut tutthug zyunkookotendul " keh njit;) kọ kwurshọ ei nitshikwuttrule, " chettenut katroogookwetiujik ko netrigwi" chitlok kirre, kọ kwịrzi kkwa tigwinidhun " kirre, tshitgichië kwikit tutzigwutaä rshọ, " kọ tshitgichië akọtigwinezhya rshọ: Chih " kwikirre akọtititinyoo elyet tte yoo kwu " tsuh nitshikwuttrule, kọ kwu tsuh tetikwi" chile, kwinidhun, kọ akotittetiya ei keh " njit, ei yoo kuttukookwichiltsei thlyeth" chyo ko chug choo kwu tsut, zut kwizyin " ha kotitinji, kọ Christ tthuị akọ tta, kwi" zyin ha koọli kwu tsut kwikit. Kwugguh " yoo ei kuttukookwichiltsei thlyethchyo ako " chug choo tthui tte kwikit kookooli, ako " elyet tte yoo tetitittaä; (kwugguh lyeh, ei " yoo kit elttsi kwu tsuh nitshikwutkwelhe " kwittelya; ei yoo tutthug Christ ttsyanut " thlih ha zyungili truttunkootutachya lyę :) " akọ Christ nuwhoh Assi zyin akọ tta zyeh " zit kookoọli, akọ tzet ukkwa, Kwugguh " yoo Christ zyin kwutthui kwu tsut elyet " tte yoo thliëtegwitli kwili ihthlud te ihthloog " kwitekit kwiyendo tsut kwittelya." 


\section{BAPTISM OF INFANTS.}

\section{TRINYUN EH NJIT BAPTISM KWUNTLUKWANJI.}

I Trinyunkthut zyunkooli baptize tunetunja kenjit gikhyi kwiyetshi tai akotetatinja. Ei ggut Etunetle choh kwu ttsut kwititititshi tle, einut trinyun negili kwah nugututazya nichin kwineinitinja ket kooei. Ako gikhyi zut niheih tai akotititenja:

YE'T chih trinyun chuttuị baptiznitunja, 1 ukkwa rshọ?

9 Ukkwa kwitizyunginyoo chi : azyuk kootle gikkhyi akọtititenja :

NUWHET tunyinutudhun, kwugguh yoo I tinjih tutthug troogwandyoh ha zyunkoọli : ako Christ nuwhoh Assi akotinyoo: Elyet ihthlud Vittukoochanchyo kookukwadhud kwi zit nitte tahah. Choo kwu ttsun akọ Chunkyo Rsotitinyoo ttsut kechid vuh koọị rzị : akotunnuwhoh chihnyoo Vittukoochanchyo Kwittyi vuh tsuh zyunguttutohchya nuwheh $\mathrm{Ku}$ kwadhud Jesus Christ kirre vih zyooggutdichi chintsi kwu ttsut chih trinyun kooh nuttlukootazya nuttlukwutandulh ei kwizyin kooli kirre shọ yitiyin: choọ ha akọ Chunkyo Rsotitinyoo ha baptize, vih tunetunja, a ako

a Koohtunetunja, Kwuntunetunja. 
Christ thlachya rsotitinyoo tet otetundulh ${ }^{2}$ ako kooh tet ihthlug trigwutundai tattsya.

I Azyuk kootle gikhyi alootititenja:

\section{Zyungutochya.}

TIHLACHI ha nichinttai akọ sheggu Vit1 tukoochanchyo tinichyo nih zyooggutdichi chob kirre Noah ako vih zzekkọ k wassittrih choh zit choo kirre elyet rsyetetkungichilya kenjit: ako Israel kkyinut nih tsyahnut rsittuttsut zyunkoohniniuk choo choh chithitsik nukuggu ei yoo kirre nih ttsun Baptism rsikotitinyoo kaönattun: akọ Jesus Christ nih Tinji vet tinutudhun baptize nitunja kirre Jordan kwinjik Choo rsotitinyoo dhantsei troogwandyoh ukwutattrhya kwu tsut kukoonatun: Nih tsut zyungittinichi nih zyooggutdichi chintsi lirre rsinichi ha chih trinyun tunaya vuk anttrhya, ${ }^{\text {b }}$ ako rsotitinyoo nyantsi ${ }^{c}$ Chunkyo Rsotitinyoo kirre: nih vecho kwu ttsut vil titinji ${ }^{\text { }}$ Christ thlachya tet ttrih choh kittukoonchyo otetundul :e ako kwikyinjigwizhit ha yinjichintluth : ${ }^{\S}$ nokonyoo kirre ssonili,g ako chettigwinidhun ha nichinttai, ${ }^{\text {h }}$ kwautseinukwattshit kwu nun kwu ttsut tyit kechoozhit ${ }^{\mathrm{i}}$ huịko tsut sheggu kwundai kthoikit kwu tsut nutahah $^{\mathrm{k}}$ azyuk nyah kukwutadhud ${ }^{1}$ sheggu

a Kotetundulh, zyunkotetundulb.

b Zyunkoohtunaya akọ zyunkoohanttrhya.

c Zyunkoohnyantsi. d Koohtitinji, kwuntitinji. e Kotetundul, zyunkotetundul.

f Yinijigichintluth, zyunyinjigichintluth. g Ssogili, ssozyungili. h Nugichinttai, nungichinttai. i Kegichoozhit, kungichoochi. k Nuguteul, nugutetal.

1 K.ukook wutadhud, kunkookwutadhud. 
kwu tsut nuwheh kukwadhud Jesus Christ kirre. Amen.

THLACHI ha nichinttai akọ sheggu Vit- ; 1 tukoochanchyo tinichyo kooh tsuh trunyundai tutthug einut ekoochichyo kunkithiyin, kooh tsuh trininji tutthug einut nih tsuh neginchya kooh tsuh truntindai kenjit, kwikyinjichinut kooh kwundai, akọ etshị kwu ttsut nuuukwalye ịlị: Nih tsut zyungittinichi chih trinyun eh njit Baptism rsotitinyoo kwu tsuh niheiha vih troogwandyoh ekootichya kotendul b kwunkyo zit kwinukwitilye kirre. Kukwa adhud oninjic nittso khenitteitinittuei nih Tinji $\mathrm{kwintluth}$ vet tinu. tudhun kirre, akọtitịnyoo: zyunotuwhohkthut, akọ kuntowhohya; koọkkunnohtyi ako kunkwutoha; Kunnulutohnei akọ nuwhoh kunuttutuneta: kwikit tukoonchyo nuwhun choog zyunochitukthut nuwhuntlunili: nuwhun kcokkunnitityi kunkoä; nuwhun kunnuluchanei nuwhet kiitininili; chih trinyun nih zyeh kug kwu ttsut kwuättrhya ttsut sheggu sso koọlị kotendul, ${ }^{d}$ akọ sheggu tsut kookukwadhud kwu tsuh nutatah e ei yoo khenitteitinittuei nuwheh Kukwadhud Jesus Christ kirre. Amen.

\section{ब1 Akotai tinjih nugututazya, akọ gikhyi akotititenja:}

Kwunduk nirzị kwi kinjik Mark Rsotitinyoo kirre uttunnututluth ihthloogchotyin

a Nigịo, neginchya. b Kookotendul, zyunkookotendal.

c Kohninji, zyunkohninji.

d Kookotendul, zyunkookotendul.

e Nuguteul, nugutetah.

56 
vih kuäzye zit, ako ihthloogchotyin tsut ttyig vikuazye tsul kwu ttsut zyunodhudhohtshei:

TRINYUNKTHUT Christ tsut nuginilye 1 kwuntitendyah kenjit : akọ vih kaönattunnut ei nukoginilyekthut zyunegigichokhe. Ko Jesus kwunayin taị elyet ssöilyah, akọ kooh tsut akotitịnyoo: Trinyunkthut seh tsuh kidichya tunkovohthun, akọ kinuntukoohtunohun rshọ: kwugguh yoo Vittukoochanchyo kookukwadhud einut kit tunginchyo enjit koọlị. Thlih ha akọtunnuwhakhnyoo: Chootyin tte Vittukoochanchyo kookukwadhud elyet trinyun kit otethundul elyet kwi zit nitte ttahah. Akọ nukoohchịli, kooh kug nunchilnjik, akọ kweh njit kwịrzị gịkhe.

ศ Kwunduk nirzi kwititititshi tle, gikhyi chih kinjik ha Kwunduk nirzi kko gitekhyah.

$\mathbb{W}_{\text {nirzi zit Christ nuwhoh Assi vih kinjik }}^{U \text { it tunyinutudhun, chih Kwunduk }}$ zyunkwutohtthuk nittso kuttukoochildhud vuh tsut nitritelya: nittso einut zukkwunkntäe ko@kunkinjichoza; nittso tinjih tutthug tsuh gikhe kooh rsittuchanchyo kwikit tunkwutedhud. Zyunyinjikwunohyin nittsọ tettiyin kirre vih chehrsikoonchyo kunkoniltyị : Kwugguh yoo nukoohchịli, kooh kug nichilnjik, akọ kweh njit kwịrzi gịkhe. Azyuk kwugguh yoo nitsye ndo ninjiowhohchi rshọ, Kọ thlạchi ha kwikyinjohchi chih trinyun thiui rsinichi ha otitetundul: ${ }^{a}$ vih chehrsikoonchyo ha niyitutachya; sheggu kwunda:

a Kokitetundul, zyunkotitetundul.

b Nukotutachya, nukotitelya. 
ttsun ssokoọli yih ntlututaä a ako sheggu tsut vih kookukwadhud kwi zit kwitekit yih ntlukootuteä. ${ }^{b}$ Azyuk kwuggut Kwikyinjinuwhahchye zyeh kug nuwhoh Ttyi vih chehrsikoonchyo chih trinyun tsut, vih Tinji Jesus Christ kirre akọtitịnyoo : akọ elyet nitsyẹ ndo ninjiidhidichi ei yoo chih nuwhoh koottrid rsinichi koọli chehrsikoonchyo ha kwunayin chih trinyun vih kwinenyitinja rsotitinyoo kwu tsuh ninyatshi: ${ }^{c}$ kwikyinjigwizhit ha akọ keịnjitchoeị ha huị vuh ntluntutotuë, akọ akọtuntotinyoo:

THLACHI ha nichinttai akọ sheggu Vit1 tukoochanchyo tinichyo, nuwhot Ttyi zyeh kug, huị nih ntluntechituë chetukotithinyoo ha ssoịlị nih chehrsikoonch yo kazyunitundai akọ nih kyinjidichi: Chih kakwutundai nuwhoh zit kwiyendo tsut zyunkwantsi, ako chih kwikyinjigwizhit nuwhoh zit nugwichinttai ha nunchilye sheggu kenjit. Chih trinyun Chunkyo Rsotitinyoo vuh ntlunyantshi, ${ }^{\text {, chun }}$ vih nukwitetilya, ${ }^{\mathrm{e}}$ kenjit, ako sheggu tsut kwuchassi tettiya: ${ }^{\mathrm{f}}$ nuwheh Kukwadhud Jesus Christ kirre, ei yoo nyah kwundai ako nyah Kukwadhud nun akọ Chunkyọ Rsotitinyoo, chooggoo ako sheggu kenjit. Amen.

I Azyuk kootle gikhyi kwittyi nilikthut ako kwuhun nilikthut akotetanjah :

a Kooh nittlukootuteä, kooh nittlukootitelya.

b Kooh nittlukootuteä, kooh nittlunkwututeä.

c Nukoohnyatshi, nunkoohvachye.

d Kooh nittlunyantshi, kooh nittlunnyantshi.

e Kooh nukwitetilya, kwrn nuk witetilya.

f Gitettisa, zyurgitettiya.

58 
NWHET tunyinutudhun chih trinyun tzet 1 nunohtshi ${ }^{\text {a }}$ baptize vih tunetunja ${ }^{\text {b }}$ kenjit, zyungutohti nuwheh Kukwadhud Jesus Christ ssonili chi yoohtendul ${ }^{\mathbf{c}}$ yih $\mathrm{d}$ troogwandyoh ekootelya, Chunkyọ Rsotitinyoo ha rsotitinyoo yih tatsya ${ }^{\text {e }}$ zyeh kug kookukwadhud ako sheggu kwundai yih ntlutitelya. ${ }^{\text {. }}$ Zyunkwutohtthuk thui nuwheh Kukwadhud Jesus Christ vih kwunduk nirzi kwi zit kheniteichituei tutthug chih ekoochichyo vookut zyungutohti kwuntleitelya; ei kheniteikwichituei thlih ha ukutututanduk kao kwikit titettiya. Azyuk kwuggut chih kheniteikwichituei tle Jesus Christ kirre kwelttsei chih trinyun thui thlih ha kheniteititetuä $g$ nuwhoh kirre nuwhun venjit ${ }^{\mathrm{h}}$ nunchidhohzi $i^{\mathrm{i}}$ ette nichizhit ${ }^{\mathrm{k}}$ kwu tsut kotitetundul ${ }^{1}$ chunkyo trandyoh, tutthug vih koottrid ha akhezyunkotitenja, ${ }^{m}$ ako Vittukoochanchyo kinjik sheggu kwikyinjitedha ${ }^{n}$ ako vih tug'witetuggun kookukwathut ta ukutututanduk. ${ }^{\circ}$

Azyuk kwuggut nuwhoh telhkhut,

[YET, chih trinyun vooh rzi zit, chunkyo $U$ trandyoh, tutthug vih kootrid ha akhe-

a Nukoohnohtshi, nukoohnohthli.

b Kooh tunetunja, kwun tunetunja.

c Koohtendul, zyunkoohtendul.

d Kooh, koohzyun.

e Kooh tatsya, zyunkooh tatsya.

x Kooh nittlutitelya, kooh nuttluntitelya.

$\mathrm{g}$ Kheniteigitetuä, kheniteiguttutazya.

h Kweh njit.

$\mathrm{k}$ Nigichizhit, nigichichye.

i Nutohthut.

I Kookotitetundul, zyunkotitetundul. m Akhezyukookotitenja.

n Kwikyinjigitedha, kwikyinjigueta.

- Ukutugututanduk, ukuntugutanduk.

59 
zyunkochịnyoo, chih nunh kug ttsun zyo kwichile ako kwichịttsi, tutthug tinjikwitizhit koọkkuttigwinidhun ha, ako kwutthuị ttsun kwitinjikwitizhit elyet kwikit tutudhịdha, akọ elyet kwíkit nih kwutinethua?

Kwiti kwi kinjik. Tutthug akhezyunkochihnyoo.

\section{Ochikkud.}

ITTUKOOCHANCHYO koọli Kwittyi
thlachi ha nichinttai zyeh nunh ha eltsei lyet kwikyinjinizhit?

Ako ihthloog rzị vih Tinji Jesus Christ nuwheh Kukwadhud? ako Chunkyọ Rsotitinyoo kirre tinjih ulttseị : nichid Mary ttsut vih kwilih; Pontius Pilate tegguk huinjithudhut, gulttsuk, ninidhut ako vuh nanjik: kwiyezyuggu kwu tsuh chozye, ako nekuddi trinzit kendo ttrin zit thunkit kwuttsun ninelikhyin: zyeh zyo chozye, ako Vittukoochanchyo Kwittyi thlạchi ha nichinttai, nandaitsei nyoti; ako azyuk kwu ttsut gunutetik nunh kwilikwutedha taị, kwundaikthut nininchikkthut ha untoututatri kenjit?

Akọ lyet kwikyinjinizhit Chunkyọ Rsotitinyoo kooli ; thlachya rsotitinyookthut nikyo zyunkooli ; rsotitinyookthut nichya zyunkwitili ; troogwandyoh ekootichya ; kwizyin nunukwutalye, akọ sheggu kwundai etshị tlẹ?

Kwiti kwi kinjik. Chih tutthug tsenja tsut kwikyinjihshit. 


\section{Gikhyi.}

TET tinjinitizhit chih kwikyinjigwizhit $\mathcal{U}$ zit baptize nih tunetunja?

Kwiti kwi kinjik. Kwikit tìnjisihzhit.

\section{Gikhyi.}

A ZYUK kwuggut lyet Vittukoochanchyo A yinji rsotitinyoo ako vih tugwitetuggun kookukwathut ha ukutạnduk, akọ kwikit tịhah tạthoog kwindai kwu tsut?

Kwiti kwi kinjik. Akọtinihthun.

I Akotai gikhyi akotititenja.

VITTUKOOCHANCHYO nyeh rsikoonchyo, akọtinidhun chih trinyun zit Adam tinyanchi kwikit vuh nutandul, tinjih kechid vih zit nuttutedha. Amen.

Akọtinịdhun kwutthuị kwu ttsut chettigwinidhun vih zit nutedha, ako tutthug ekoochichyo Chunkyo ttsun koọl vih zit kwutendai ako tinezye. Amen.

Akọtinịdhun ttuih tettiya chunkyọ trandyoh, nunh kug ako kwutthuị kwu tsut kwutugtichichyo ako kwu tsut kukwutadhud. Amen.

Akọtinịdhun chootyin tte tzet nyih ntludhitshi nuwhoh koottrid kirre, zyeh kug kwu ttsut kwiyinji nirzi ha vih zit tugwitutunaä, ako sheggu kwu tsut vuh ntlututazya nih zyooggutdichi kirre, O Kukwadhud Vittukoochanchyo ssoili, sheggu kwindai, ako tutthug ekoochichyo kwu tsuh kukwạdhud sheggu kenjit. Amen. 
THLACHI ha nichinttai, sheggu kwundai Vittukoochanchyo tinichyo, nih Tinji Jesus Christ kwintluth vettinutudhun, nuwhot troogwandyoh ekooteta kenjit vih ttsyeko kwichile kwu ttsut choo tta ha neichonja; akọ tuh kaönattunnut kutukoochildhud tutthug kutchinnut tsuh gutetah akọ kunukotunachya, ako baptize gutunenja, Kwittyi, Kwitinji, Chunkyo Psotitinyoo ha koh rzi zit: Kwinyayin nih tsut zyungittinichi, chih ehthluggoonidichyakthut kooh gichinchi ; chih choo rsotitinyoo nyantsi troogwandyoh ukootatrha kookukwitunatya kwu tsut; ako akọtinịdhun chih trinyun chooggoo kwizit $v i h^{a}$ neitunetunja nih chehrsikoonchyo thlạchi ha kotendul b ako nih kkyinut kwikyinjichyenut akọ uttoutitinyookthut tet sheggu telya nuwheh Kukwadhud Jesus Christ kirre. Amen.

I Azyuk kootle Gikhyi trinyun nututachya, akọ kwiti nugichilzikthut tsut akọtitenja:

Chih trinyun onirzi.

9 Ako tle trinyun kooh kyit yootirzi, akotititenja:

N Baptize nyih nihnja Kwittyi, ako Kwitinji, akọ Chunkyọ Rsotitinyoo koh rzi zit. Amen.

T Azyuk kootle Gikhyi akọtititenja:

CHIH trinyun onitinji Christ vih thlachya $U$ tet, akọ kwinitituëkoogooteyin ha vih kwitilhtshi, kakwutetundai kenjit chooggoo
a Kooh, kwun.
b Kookotendul, zyunkookotendul. 
kwu ttsut elyet trituttenji, Christ gulttsuk kwikyinjigwizhit ket kwutanduk, ako yinjikwichintluth ha Christ vunuäe zuggu, troogwandyoh, nunh kug, ako chunkyo trandyoh kwu tsut niitsitathut; akọ Christ vih nuve kyinjizhit akọ ultsun telya tạthoog kwutendai kwu tsut. Amen.

\section{Tा Azyuk kootle Gikhyi akọtititenja :}

NUWHE' $\mathrm{C}$ tunyinutudhun chooggoo zyun1 kwunohyin chih trinyun vih nukwitili, a ako Christ thlachya tet kwunkyo nili ${ }^{\text {b Vit- }}$ tukoochanchyo thlạchi ha nichinttai huị vuh ntluntutotuë chih ekoochichyo kwịrzị kenjit; ako kwittri ihthloog vuh tsuh zyungutohchya, chih trinyun tathoog kwutendaic chooggoo koogwinyäei kit kwutendai.d

T Akọ tle tutthug nuntshikwuttrule, akọtititetinja:

NUWOT Ttyi zyeh zit nih kwilnjik. I Nyooh rzi chootsi. Nih kookukwadhud nuvizi. Tinịdhun nunh kuggu tugoochyo nittsọ zyeh zit troogwatsi kwi kit. Chih ttrin zit nuwhun enyantsit ttrin ndo thloogootunazya nuwhoh ssih. Ako nuwhot troogwandyoh nuwheb nnkoochịli nittsọ kooh ttsut unkoochitili einut troogwandyoh nuwhet tungittiyin. Ako kookukwutundai kwu tsut nuwhoh tunọe rshọ; ko troogwandyoh kwu ttsut nuwhoh yunninji: Kwugguh yoo

a Kooh nukwitili, koohzyun nukwitili.

b Gili, zyungili.

c Kookwutendai, zyunkookwutendai. 63

d Kookwutendai, zyunkookwutendai. 
nih ttsun nili kukwadhud, ttuih, akọ nindelh sheggu kenjit ako sheggu kenjit. Amen.

\section{Akọtai Gikhyi akotititenja :}

K WITTYI kwintluth nyeh rsikoonchyo hui I nyih ntlunchituë kwittri ha ssoịlịi chih trinyun vih nukwitilị dhantsei gelyẹ, Nyuh Nkyọ Rsotitinyoo kirre, nih kkyi eh njit ochidhitinjik, akọ nih thlachya rsotitinyookthut tet nạntshị. Akọ chetukotithinyoo ha nih tsut zyungittinichi akotiyitinizhya chi, troogwandyoh kwu tsut nutedha, ako tshoogoochöe kwu tsut kwutendai, akọ Christ vih tshị zit ha vunanji, tinyanchi gutatsuk, ako troogwandyoh kookwizyin thlạchi ha rsyetekoogootutathlye a ako nih Tinji ninidhud veh koochattshit, ${ }^{b}$ vih nunukwalye thui vuh kootutattha ${ }^{c}$ akotukoonchyo huiko tsut tutthug nih thlachya rsotitinyookthut ha nih kookuwadhud sheggu kwu tsut koọl kwi zit telya; nuwheh Kukwadhud Christ kirre. Amen.

9 Ako tle tutthug nungichilzi, Gikhyi kwiti nichilzikthut tsut akotititenja:

K WUGGUH yoo chih trinyun, nuwhun I veh njit nuchidhohzye kirre kheniteichittueị, chunkyo trandyoh tutthug vih koottrid ha akhezyunkotitenja, Vittukoochanchyo kyinjitedha, ako yih kukwutathut: kunnutohndai lyẹ, nuwheh njit kookooli zyunkwutunohya chih trinyun kouttunachya, ettet $\mathrm{kwu}$

a Rsyetekoogookwututathlye, rsyetekungookwututathlye.

b Kweh koochattshit, kwun kootatthut.

c Kweh kootutattha, kwun kootutattha. 
tsut kudhun kikyitandul, tugwantluth kiuji nichinttai, kheniteikwichittuei ha keh kwanduk ha kechikwiyin tzet kweltsei nuwhoh kirre. Akọ chih ekoochichyo kwiyendo tsut kwirzi. katetundai kenjit, vuh tsuh zyungutohkhya Vittukoochanchyo kinjik gitshigilili kotudhatsha ; ako kwiyendo tsut zyunkwutunohya ei yoo kwikyinjigwizhit, Kukwadhud vih gichinchi, ihthloogchotyin tugwitetuggun ha tinjih kinjik zit kikyitandul, ako tutthug chizi ekoochichyo chitti tte Christ ttsun nili ettelyẹ katetundai akọ kwikyinjitedha vuh nkyọ rsyetelya kwu tsut; ako chih trinyun kwịrzi tsut tikyittadha ${ }^{\text {a }}$ kwịrzi tsut ako tshoogoochöe tsut kwutendai ; ${ }^{b}$ sheg kunudhundai ${ }^{c}$ ei yoo kwinenyitinja kookunuwhohnatye nittso tsut zyunkwutitundai; ei yoo nuwhoh Assi Christ nittso kwundai kwikit tituta, ako vih kyit zyunnuwhoh ttatsya: nittso nuwheh njit ninidhud ako ninelikhyin. kwikit tukoonchyo nuwhun tte, nuwhun neinyithunja, troogwandyoh kwu tsut nuttitutah akọ tshoogoochöe tigwittiyin kwu tsut nutituta; sheggu khuinjjikwuächi tutthug nuwhoh yinji kwịrzi kkwa, ako ndothloogootunazya kwiyendo tsut kwịrị kookwundai tikyitituta.

\section{T Azyuk kootle chih chun akotititenja:}

$\mathrm{K}$ UNNOUHNDAI chih trinyun gikhyi I choh tsut nuttachya ${ }^{d}$ yih kug nunulutazya, kwikyinjigwizhit kwi kinjik, Kukwa-

a Tikyitkotadha, tikyitkotata.

b Kookwutendai, zyunkookw utendai.

c Kunugandai, kunnugandai.

d Nukoohttachya, nunkoohttachya. 
dhud vih gichinchi, ako tugwitetuggun ihthloogchotyin, tinjih kinjik zit, katetunda $i^{\text {a }}$ tai, ako kwiyendo tsut koutunachya ochikkud etenetle, ei keh njit kuttukootattshe.

- Ettelye kakwitundai Vittukoochanchyo kinjik kirre, trinyunkthut vuneinyithunja, nigininchik troogwandyoh tungittihin kwiyetshi, ettethlih ha zyungitizi.

\section{BAPTISM OF ADULTS.}

\section{TIKYINCHIKTHUT EH NJIT BAPTISM KWUNTLUKWANJI.}

बा Tikyinchikthut zyunkooli baptize kwun tunetunja kenjit, gikhyi kwiyetshi tai akotettatinja. Azyuk kwuggut etenetle choh kwu ttsut kwititititshi tle, einut nichin tte Baptism ket kooei zut nugututazya. Ako gikhyi akotititenja :

TUWHET tunyinutudhun kwugguh yoo 1 tinjih tutthug troogwandyoh ha zyunkoọlị (akọ ei kwutthuị kwu ttsut vuh koọli kwutthuị nilị) ako einut kwutthuị zit zyungịlị elyet kunkidhun Vittukoochanchyo sso ttsun zyungutatsya, ko troogwandyoh zit zyunkookwundai, akọ kwịlyeị kwịrzị kkwa tungittiyin : ako Christ nuwhoh Assi akọtitịnyoo: Elyet ihthle kidhun Vittukoochanchyo kookukwadhud kwizit nitte tahah, vih nukwitili rzi choo ha Chunkyo Rsotitinyoo ha: nuwhoh chisuhkthut Vittukoochanchyo kwittyi zyun66

a Kagitetundai, kazyungutetundai. 
guttunohchi nuwheh Kukwadhud Jesus Christ kirre, vih chehrsikoonchyo kirre chithnut kooh nittlunkootitelya a ei yoo kwutthuị kirre shọ kittiyin $;^{\mathrm{b}}$ choo ha ako Chunkyo Rsotitinyoo ha baptize kwuntunetunja ${ }^{c}$ ako Christ thlachya rsotitinyookthut tet zyunkotetundul, ako trigwutundaikthut zyunkoohttatsya:

I Ako tle gikhyi akotititenja :

Zyungutochya.

( $($ A Akotai thlachya tutthug nuntshikwutguttä̈.)

THHLACHI ha nichinttai ako sheggu Vittu1 koochanchyo tinichyo nih zyooggutdichi choh kirre Noah vih zzekkọ ha zyunkwạssi ttrih choh zit choo kirre elyet rsyetetkungichilya keh njit; ako tte Israel vih kkyinut nih tsyahnut rsittuttsut zyunkoohniniuk choo choh chithitsik nukuggu ei yoo kirre nih ttsun baptism rsikotitinyoo kaönattun; ako nih Tinji Jesus Christ vettinutudhun baptize vih nitunja kirre Jordan kwinjik choo rsotitinyỏo kwudhantsei troogwandyoh ukwutattrhya kwu tsut kukonatun: Nih tsut zyungittinichi nih zyooggutdichi chịttsi kirre rsinichi ha chih nih ultsunnut zyunkoohtunaya; kwun anttrhya ${ }^{e}$ ako rsotitinyoo zyunkoohnyantsi Chunkyo Rsotitinyoo kirre, nih vecho kwu ttsut kwun titinji, Christ thlachya tet trih choh kit tukoonchyo zyunkotetundul; ako kwikyinjigwịzhit kirre zyunyinjigichintluth, nokonyoo kirre ssozyungili, ako chettigwinidhun ha nungichinttai, kwäutseịnukwatshit kwu nunh a Nittlukootitelya. c Vih tunetunja.

b Kigittiyin, kungittiyin. d Otetundul. e Kwuh. . F Kooh. 
kwu ttsut tyit kungichoochi, huịko tsut sheggu kwundai kthoikitkwu tsuh nugutetah azyuk nyah kunkookwutadhud sheggu kwu tsut nuwheh Kukwadhud Jesus Christ kirre. Amen.

IIHLACHI ha nichinttai akọ sheggu Vittu1 koochanchyo tinichyo, kooh tsuh trunyundai tutthug einut ekoochichyo kunkithiyin, kooh tsuh trininji tutthug einut nih tsuh neginchya kooh tsuh truntindai keh njit, kwikyinjichinut kooh kwundai, akọ etshị kwu ttsut nunukwalye ịli : Nih tsut zyungittinichi chihnut eh njit nih Baptism rsotitinyoo kwu tsuh neginchya, kooh troogwandyoh ekootichya zyunkookotendul kwunkyo zit kwinukwitilye kirre. Kukwạdhud kooh tukwạji nittso khenitteitinituei nih Tinji $\mathrm{k}$ wintluth vet tinutudhun kirre, akọtitinyoo: zyunotuwhohkthut ako kuntowhohya : kookunnohtyi akọ kunkwutoha; kunnulutohnei akọ nuwhoh kunuttutuneta; kwikit tukoonchyo nuwhun choog zyunochitukthut nuwhoh ntlunili; nuwhun koọkunnitityi kunkoä; nuwhun kunnuluchanei nuwhet kiitininili : chihnut nih zyeh kug kwu ttsut kwuattrhya ttsut sheggu ssokoọli zyunkookotendul, akọ sheggu tsut kookukwadhud kwu tsuh nugutetah ai yoo kheniteitinituei nuwheh Kukwadhud Jesus Christ kirre. Amen.

I Akọtai tinjih nugututazya, ako gịkhyi akotititenja:

K WUNDUK nirzị kwi kinjik John RsotitiI nyookirre attunnututluth ttyid vih kuazye

a Nugutëulh.

68 
zit, trootshid vikuazye ttsul kwu ttsut koogwinyäei.

Pharisinut tet tinjihihthlẹkwilịh Nicodemus vazi, Jewnut kootshid tet ihthle ịlih. 2. Ei yoo ttodh Jesus tsuh ninizi akotiyahnyoo: Rabbi, kazyunitundai kaönattun ịlị Vittukoochanchyo ttsut ninizi; kwugguh yoo chih koogooteyin tinitiyin elyet ihthle kidhun titettiya Vittukoochanchyo yah nili rzi. Jesus kwitị gịkhe akotiyahnyoo: Thlih ha, thlih ha, akotinyakhnyoo ; Tinjih kechid vih nukwitili kkwa chi, Vittukoochanchyo kookukwadhud shọ nayin. Nicodemus akọtiyahnyoo: Nittsukwaun tte tinjih vih nukwitetilya tinyanchi dhitlit taị ? Lyet rshọ kenendo tuh hun tsut zit netizhit, ako kenendo vih nukwitetilya? Jesus kwitigikhe: Thlih ha, thlih ha, akotinyakhnyoo : Tinjih choo ttsun Chunkyọ Rsotitinyoo ha vih nukwitili kkwa chi, shọ Vittukoochanchyo kookukwadhud kwizit nitte taheih. Ei yoo kwutthuị ttsun vuh koọlị kwutthuị nilị; akọ ei Kwunkyo ttsun vuh koọli kwunkyọ nilị. Kuninjiọdhud rshọ kwugguh yoo akọtinyakhnyoo: tinjikwitizhit lyẹ nuwhoh nukwitetilya. Attrui attrui nichin kookwinidhun, ako kwitezyoo kootinyitthuk; kọ elyet kadhitinjik nichin tte kwu ttsut nugoochichya, ako nichin tte kwu tsut tidichya: kwikit tukoonchyo titegookwinyachyo Chunkyo ttsut kwi nugwitili.

\section{बा Akọ tle gikhyi akọtititenja :}

WUWHET tunyinutudhun, chih Kwunduk 1 nirzi zit nuwhoh Assi Christ kinjik 69 
zyunkwutohtthuk nittsọ tinjih choọ ha Chunkyo Rsotitinyoo ha elyet vih nukwithili chi, elyet kidhun Vittukoochanchyokookukwadhud kwi zit nitte tahah. Ei kwikirre zyunyinjikwinyayin nittso chih kuttukootattshe kwintluth ket koọli nichin kookwudhun kuttukootandul. Akọ zyeh zyọ nethizi kwu tsuh nyakwuth kwiyetshi (nittsọ Mark rsotitinyoo kwunduk nirzi kwizit zyunkwitichititshi) tuh kaönattunnut tsuh kuttukoochildhud, akọtitịnyoo: Nikyo nunh kug kwutohta, akọ kwunduk nirzị tuthug kwunduikthut tsut keh zyunkohnduk. Ei kwikyinjizhit akọ vih baptize nitinja tetizi ; kọ ei elyet kwikyinjoshit uneinjikwutetudha: ei yoo tthui kookunnuwhoh natye nittsọ ei kwikirre kwintluth khunkwazye. Ei ggut Peter rsotitinyoo, chileh, trootshid kwunduk nirzi keh kwanduk taị lyeịnut koh ttri kunttlyäuttanei akọtungiyahnyoo ako chizi chilehnut thui tsut akọtungitịnyoo : Tinjihnut kwuluggukthut ha, ttuntitiya? Peter kwiti gịkhe akotunkovahnyoo: kineinjitowhohchi, ako nuwhot tutthug nuwhun baptize nyootunja troogwandyoh ekooteta kenjit, akọ Chunkyo Rsotitinyoo, ei yoo ozi nili, zyunotohndul. Kwugguh yoo ei kheniteikwichittuei nuwhoh tsut ako nuwhoh kkyinut tsut, akọ einut nizhid zyungịi tsut zyunkoọlị, akọtinyachyonut yoo Kukwadhud nuwhoh Vittukoochanchyo kookkungutekhya. Akọ chizi kinji lyei ha kooh tsuh zyungịkhe, akọtitinyoo: chih nizyoonedichi ttsut zyunuttutohzi. Kwugguh yoo (nittso ei ehthlooginchi chileh kwuneäggoo keh kwanduk) Baptism tthui 70 
chooggoo nuwhassi (elyet tte yoo kwutthui kwu rshrui ekootichya, ko uttakakwutundai nirzi-kwi kwiti gịkhyi nili Vittukoochanchyo tsut) Jesus Christ vih ninukwalye kirre. Azyuk kwuggut nitsye ndo ninjiöwhohchi rshọ, kọ thlạchi ha kwikyinjohchi, chihnut rsinichi ha zyunotendula thlih ha kineinjikidichi, ako kwikyinjigwizhit ha gih tsuh ninchya; kooh troogwandyoh ekootelya, ako Chunkyo Rsotitinyoo kooh nittluntachye; sheggu kwundai kwi ssokoọli kooh nittluntitelyạ, akọ sheggu kwu tsut vih kookukwadhud kwizit zyunkotitetitsha.

Azyuk kwuggut nuwhot Ttyi zyeh kug vih rsinichi chihnut tsut kwikyinjidichi, vih Tinji Jesus Christ kirre akotitinyoo: kwikyinjigwizhit ha ako keinjitkwichöeị ha huị vuh ntluntutotuë, akọ akọtuntotinyoo:

THLACACHI ha nichinttai akọ sheggu VittuI koochanchyo tinichyo, nuwhot Ttyi zyeh kug, chetukotithinyoo ha hui nyih ntlunchituë, kwugguh yoo sso ịlịh nih chehrsikoonchyo kazyunitundai akọ nih kyinjidichi : chih kakwutundai nuwhoh zit kwiyendo tsut zyunkwantsi, ako chih kwikyinjigwizhit nuwhoh zit nugwichinttai ha nunchịlye sheggu kenjit. Chihnut Chunkyo Rsotitinyoo kooh nittlunyantshe, koohzyun nukootetilya keh njit, ako sheggu tsut kwuchassi zyungitettiya, nuwheh Kukwadhud Jesus Christ kirre, ei yoo nyah kwundai ako nyah kukwadhud nun ako Chunkyọ Rsotitinyoo, chooggoo ako sheggu keh njit. Amen.

$$
\text { a Kotendul, otendul. }
$$


T Azyuk kootle Gikhyi einut kwun neitunetunja tsut akotititenja:

NUWHET tunyinutudhun tzet nunnohkookuttunyinoothun, zyunkwutohtthuk nittso thlachya zyunkigiteti, nuwheh Kukwadhud Jesus Christ akọteyitinezhya zyunnuwhoh tendul ako kwịrzị tsut tunnuwhoh tayah, ako zyeh kug kookukwadhud nuwhoh ntlukootitelya, sheggu kwundai ha. Zyunkwutohtthuk tthui nuwheh Kukwadhud Jesus Christ vih kinjik rsotitinyoo zit kheniteichittuei tutthug ei ekoochichyo ggut zyungutohti ${ }^{\text {b }}$ kwuntlukootitelya: ei kheniteikwichittuei, ettelye ukututanduk ako kwikit titettiya.

Azyuk kwuggut chih kheniteikwichittuei tle Christ kirre kwelttseị, nuwhun tte thlachi ha kheniteitutohzya ${ }^{c}$ kooh nda chihnut nugichilzi, ako tutthug chih thlachya, chunkyọ trandyoh tutthug vih koottrid ha, akhezyunkotutohnjad ako sheggu tsut Vittukoochanchyo kinjik rsotitinyoo kwikyinjitohta, ako kookukwathut ha vih tugwitetuggun ukuntututohnduk. e $^{-}$

I Akotai Gikhyi einut baptize kwun tunetunja kootugookwinyachyo tsut, kwikit zyunotutakhut:

I YET chunkyo trandyoh tutthug vih koo1 ttrid ha akhezyunkochịnyoo, nunh kug zyo kwichile kwichinttsi ha, tutthug tinjikwitizhit koọkkuttigwinidhun ha, akọ kwutthuị

a Nunoheth.

c Kheniteitutowhoha, kheniteititintuä.

d Akhekotitinja.

e Ukutututanduk. 
$\mathrm{kwu}$ ttsut tinjikwitizhit, elyet kwikit tutudhịdha, akọ elyet kwikit nih kwitinethuä ?

Kwiti kwi kinjik. Tutthug akhezyunkochibnyoo.

\section{Ockikkud.}

JTITUKOOCHANCHYO koọi Kwittyi thlanchi ha nichinttai, zyeh nunh ha eltsei lyet kwikyinjinizhit ?

Akọ ihthloog rzi vih Tinji Jesus Christ nuwheh Kukwadhud? akọ Chunkyo Rsotitinyoo kirre tinjih ulttsei; nichid Mary ttsut vih kwịịh; Pontius Pilate tegguk huinjithudhut, gulttsuk, ninidhud, akọ vuh nanjik; kwiyezyuggu kwu tsuh chozi, ako nekuddi ttrin zit thunkit kwu ttsut ninelikhyin; zyeh zyo chozi, ako Vittukoochanchyo Kwittyi thlanchi ha nichinttai, nandeitsei nyoti: ako azyuk kwu ttsut gunutetik nunh kwilikwutedha tai, kwundaikthut nininchikkthut ha untoututatri kenjit ?

Ako lyet kwikyinjinizhit Chunkyọ Rsotitinyoo koọli : thlachya rsotitinyookthut nikyọ zyunkooli ; rsotitinyookthut nichya zyunkwitili ; troogwandyoh ekootichya: kwizyin nunukwutalye, akọ sheggu kwundai etshị tlẹ ?

Kwiti kwi kinjik. Chih tutthug tsenja tsut kwikyinjihshit.

\section{Ockikkud.}

T YET tinjinitizhit chih kwikyinjigwizhit 1 zit baptize nyih tunetunja?

Kwiti kwi kinjik. Kwikit tinjisihzhit.

73 


\section{Ockikkud.}

\section{A ZYUK kwuggut lyet Vittukoochanchyo A yinji rsotitinyoo, ako vih tugwitetuggun kookukwathut ha ukutạduk, akọ kwikit tịhah tạthoog kwindai kwu tsut?}

Kwiti kwi kinjik. Akọtinihthun.

\section{ๆ Akotai Gikhyi akọtititenja.}

D VITTUKOOCHANCHYO nyeh rsikoonchyo, akọtinịdhun chihnut zit Adam tinyanchi kwikit vuh nutandul tinjih kechid kooh zit nuttutedha. Amen.

Akọtinịdhun kwutthuị kwu ttsut kettigwinidhun kooh zit nutedha, ako tutthug ekoochichyo Chúnkyo ttsun koọlị kooh zit kwutendai ako tinezye. Amen.

Akọtinịdhun ttuih zyungitettiya chunkyọ trandyoh, nunh kug akọ kwutthuị kwu tsut kwutugtungichichyo akọ kwu tsuh kunkookwutadhud. Amen.

Akọtinịdhun chootyin tte nuwhoh koottrid kirre nih ntludhitshi, zyeh kug kwu ttsut kwiyinji nirzi ha vih zit tugwitutunaä akọ sheggu kwu tsut ssokoọlị vuh ntlukootutazya, nih zyooggutdichi kirre, O Kukwadhud Vittukoochanchyo ssoịli, sheggu kwindai, ako tutthug ekoochichyo kwu tsuh Kukwadhud sheggu kenjit. Amen.

TTHLANCHI ha nichinttai ako sheggu Vit1 tukoochanchyo tinichyo nih Tinji Jesus Christ vet tinutudhun nuwhot troogwandyoh ekooteta kenjit vih ttsyeko kwichile kwu ttsut chop tta ha neichonja; akọ tuh kaönattunnut 
kuttukoochildbud tutthug kutchinnut tsuh gutetah, ako kunukootunachya, akọ baptize gutunenja, Kwittyi, Kwitinji, Chunkyo Rsotitinyoo ha koh rzi zit: Kwinyayin nih tsut zyungittinichi, chih ehthlooggoonidichya kooh gichinchi : chih choo rsotitinyoo nyantsi troogwandyoh ukootattrha kookukwitunatya kwu tsut; ako akọtinịdbun chihnut choog kwi zit kwun neitunetunja thlanchi ha nih chehrsikoonchyo zyunkookotendul ako nih kkyi kwikyinjichyenut, ako uttoutitinyookthut tet kwittichithityin sheggu zyungittelya nuwheh Kukwadhud Jesus Christ kirre. Amen.

I Akotai Gikhyi einut baptize kwuntunetunja kootugookwinyachyo tsut kwu nli ha otendul, ako nittso ttoutazya yooh tutakhut, ako tle choo ha baptize yih tunenja, akotititenja ha:

N Baptize nih nihnja Kwittyi akọ Kwi1. tinji ako Chunkyọ Rsotitinyoo oh rzi zit. Amen.

\section{T Akọtai Gikhyi akọtititenja :}

CHIH tinjih Christ thlachya tet onitinji, ako kwinititue koogooteyin ha vih $\mathrm{kwi}$ tilhtshi, kakwutetundai chooggoo kwu ttsut elyet trituttenji Christ gulttsuk kwikyinjigwizhit ket kwutanduk, ako yinjikwichintluth ha Christ vunuäe zuggu, troogwandyoh nunh kuggu, akọ chunkyọ trandyoh kwu tsut nütsitathut; ako Christ vih nuve kyinjizhit ako ultsun telya tạthoog kwutendai kwu tsut. Amen. 


\section{Tा Ako tle Gikhyi akotititenja :}

TUWHET tunyinutudhun chooggoo zyun1 kwunohyin chihnut koohzyun nukwitili, ako Christ thlachya tet kwunkyo zyungili, Vittukoochanchyo thlanchi ha nichinttai hui vuh ntluntutotuë chih ekoochichyo kwịrzi kenjit: ako ihthloog kwittri ha vuh tsuh zyungutochya, chihnut tathoog zyunkookwutendai chooggoo koogwinyäei kit zyunkookwutendai.

T Akọtai tutthug nuntshikwutgutaä, akọ akọtititetinja:

NUWHOT Ttyi zyeh zit nih kwilnjik. IN Nyooh rzi chootsi. Nih kookukwadhud nuvizi. Tinịdhun nunh kuggu tugoochyo nittso zyeh zit troogwatsi kwi kit. Chih ttrin zit nuwhun enyantsit ttrin ndo thloogootunazya nuwhoh ssih. Ako nuwhot troogwandyoh nuwheh unkoochịli nittso kooh ttsut unkoochitili einut troogwandyoh nuwhet tungittiyin. Akọ kookukwutundai kwu tsut nuwhoh tunọe rshọ; ko troogwandyoh kwu ttsut nuwhoh yunninji: Kwugguh yoo nih ttsun nilị Kukwadhud, ttuih, akọ nindelh sheggu kenjit ako sheggu kenjit. Amen.

K WITTYI zyeh kug ili chetukotithinyoo kkunginịkhyi nih chehrsikoonchyo kazyuntitundai, akọ nih kyinjitituta: Chih kakwutundai nuwhoh zit kwiyendo tsut zyunkwantsi, ako chih kwikyinjigwizhit nuwhoh zit nugwichinttai ha nunchilye sheggu kenjit. Chihnut Chunkyọ Rsotitinyoo kooh nittlunnyantshi; 
chooggoo kwun nukwitilị akọ sheggu tsut kwuchassi zyunkittiyin nuwheh Kukukwadhud Jesus Christ kirre, nyuh ultsunnut sheg: gu kwu tsut zyungittelya, ako nih kheniteikwichituei kungitettiya, ei ehthlooginchi $\mathrm{Ku}$ kwadhud nih Tinji Jesus Christ, ei yoo nyah kwundai ako nyah kukwadhud ehthloogtilị zit, ehthlooginchi Chunkyo Rsotitinyoo ha sheggu kenjit. Amen.

\section{वा Azyuk kootle tutthug nungichilzi, Gikhyi akotititenja: trootshid kwiti nungichilzi- kthut tsut akotititenja :}

W WUGGUH yoo chihnut nuwhoh ndah thug vih koottrid ha akhezyunkookotitenj ${ }^{\mathrm{b}}{ }^{\mathrm{b}}$ Vittukoochanchyo kyinjiguteta ${ }^{c}$ ako gihkuntanduk; kunuutohndai nuwheh njit kookooli, kunnukotohndai, ${ }^{\text {e tugwantluth kinjih nichin- }}$ ttai ako kheniteikwichittuei, ako keh kwanduk ha kechitkwiyin chih thlachya ndah chooggoo zyunkoogweltsei, ako nuwhoh ndah nuwhun kweh njit nuchidhohzye. Ako kooh tsuh zyunguttohkhya zyunkuttetundai tsenja tsut kunkottunachya Vittukoochanchyo kinjik rsotitinyoo zit; azyuk kwuggut chehrsikoonchyo zit tikyunguteta, ${ }^{\mathrm{f}}$ ako kwiyendo tsut nuwheh Kukwadhud Jesus Christ hazyunguttetundai,g ako kwịirị, tshoogoochoë ha akọ rsittuttsut zyunkookwutendai chih nunh kuggu.
a Kheniteigichittuei.
b Akhekookotitenja.
c Kyinjigutedha.
d Gihkutanduk.
e Kunnu-tohndai.
g Hagutteundtai, hatetundai.
f Tikyigutedha. 
ब Akọ tle einut kechid baptize kwun nithunja tsut Gikhyi akotunkohtanja :

NUWHUN yoo chooggoo Baptism kirre 1 Christ nohzi, nuwheh njit ket kooli thui, Vittukoochanchyo ako Attri ha kwikkyinut zyunnuwhoh lttsei, Jesus Christ vih kyinjigwizhit kirre, ette lyẹ nittsọ Christ ttsun nilikthut gutetah chi kwi kit tohtah lyee, ako attri kwi kkyinut nittso kweh njit ket koọị : sheggu kunnouhndai ei yoo Baptism kookunnuwhoh natye nittso zyunkwutitundai thlih : ei yoo nuwhoh Assi Christ vih kyun tutitudhut, ako vih kyit tutituta ; nittso ninidhud ako nuwheh njit ninelikhyin; nuwhun tte baptize nuwhoh nyithunja, troogwandyoh kwu tsut nuttituta, ako tshoogoochoë tigwittiyin kwu tsut nutituta; sheggu tutthug nuwhoh yinji kwịrzị kkwa khuịnjikwuachi, akọ ndothloogootunazya kwiyendo tsut kwịrzi kookwundai kwikit tituta.

9 Tinjikwitizhit tutthug einut kwikit baptize kwunnyithunja nyakwuth kootle Gikhyi choh kooh lug nunulutazya: ninjikwudhud rsotitinyoo zyunkookotendul gelye. 


\section{SOLEMNIZATION OF MATRIMONY.}

\section{NIKHUT-TRINIDITI RSIKOTI- TINYOO HA TUGWAYIN.}

- Trootshid koo kwunduk einut nikhuntutunatsha trigichinchi zze kwi zit kukwutanduk nukwazi ttrin zit ttyid nikendochilzi, vvun trigikhyi tai, ko khah tsut trigikhyi tai (voun trigikhyi kooli klwwa chi), inchit tsut nekuddi etunetle choh kwu ttsut kwikit tai trigikhyi tle : gikhhyi nittso sheg tegwinyoo lkwikit tinyoo:

K UKWULHNDUK nikhut-triniditi koo I kwunduk M. N. ha kookyidetug. : Nuwheh ttenut ekoochichyo kazyunwhuhndai chi, ko ekoochichyo tshoogoochoë, chyaggut tte chih nekunnut elyet nikhut-tinethitya nikhut triniditi rsikotitinyoo ha kuzyunkwutohnduk. Chih yoo trootshid (nekuddi, ko tyiddi) kochikkud.

I Ei ttrin zit ako akotai kuttukootatshi, nikhut-triniditi rsikotitinyoo ha tugwayin gelye, einut nikhut tutunatsha trigichinchi zze lewizit nituguteta kuttu luggukthut hos ako kooh nëaggookthut ha: ako zut eh. thlooggoo nugichilzi, tinjih nandeitsei tsut, ako ttrig ttletsei gikhyi akotititenja : 
WINTLUTH nuwhet tunyinutudhun, 1 tzet chunzyunnithlichazi Vittukoochanchyo ndah, ako chih chunzyun-nithlitazi kooh ndah, chih tinjih chih trig ha nikhut-guttunetitya nikhut triniditi rsikotitinyoo zit; ei yoo yinjikwichile tigwinidhun, Vittukoochanchyo kuttugwichiltshei tinjih ịrzyoo taị, ei kwikirre kookkunnuwhoh natye nikhut-gwunati nunäi Christ vih thlachyakthut kwikidetug koolị; ei rsikotitinyoo tigwitiyin Christ kwịrzị kweltseị akọ ndotinchyo kweltseị vuh ndah, akọ trootshid koogooteyin kweltsei, Cana kwizit Galilee kthoikit; ako Paul rsotitinyoo kwịrzị kwu tsuh gịkhe, tinjih tutthug kooh tet yinjikwichile nili.

Kuttukoochilttshei, nikha kitili kenjit, kwu tsuh trigwinji, ako khukwizhit ihthle ei ihthle ttsut kitetiya lye, kwịrzi kooei taị, ako kookoonttri kooei taị. Ei rsikotitinyoo kooei chih nekunnut tzet tiginchyo choog $\mathrm{kwu}$ tsuh nugiö nikhut-guttunetitya kenjit. Azyuk kwuggut nuwhoh tet ihthle, kudhun kookuinatye ekoochichyo kooli chyaggut tte rsittuttsut nikhut guttunethitya chooggoo gokhyah, ukkwa chi tzet kootle sheg kwu tsut gokhyah rshọ.

\section{Aleo ei nikhut guttunetitya tsut tthui, akotititenja :}

NUWHET tinjisihzhit ako ehthle tsut ako1 tunuwhakhnyoo, kwitị gutohkhyah kyit, kuttukootutattri ttrin zit koogwunachut etchihtai kwi ttri tutthug kwu nukwuzug kakwutetundulh, nuwhun ihthle ekoochichyo 80 
katundai chi chyaggut tte nikhut triniditi ha rsittuttsut shọ nikhut tunowhohtya, chooggoo kohnduk. Kwugguh yoo tshoogoochoë kawhuhndai, tegwinyachiddi tte nikhut triniditi Vittukoochanchyo kinjik nitsye Vittukoochanchyo kirre elyet nikhut kinuthiti: akọ kooh nikhut triniditi rsittuttsut kwili.

I Ei nikhut triniditi ttrin zit tinjih iththle ekoochichyo tinyoo chi, chyaggut nikhut gutunethity a lye nikhut triniditi Vittukoochanchyo vih tugwitetuggun kit : akotai nikhut triniditi akhenukootitetinja, akọtai kwu tsut thlih ha kakwutetundai.

T Elyet ekoochichyo titithinyoo chi, akotai gikhyi tinjih tsut akotititenja:

MI. T YET chih trig nyuh ut eh ujit vih $\mathcal{U}$ tintiya lye, nikhut triniditi ha, nikha kwutohndai keh njit Vittukooehanchyo vih kuttukootatshe kit, rsikotitinyoo tegwichöei Nikhut triniditi zit? Lyet vettitinịzhya, et vih rsyetututanjik, et yinjitạa, et vih kutunantya eltsik tai ako rsyekwundai taị ; akọ chizi tutthug akheöchịnyoo, attun rzị vuh tsut uttikunyạtyi, tạthoog ehthle tsut kwutohndai?

of Tinjih akotititenja:

Kwikit titihsiya.

T Akọtai gikkhi Trig tsut akọtititenja:

$N$. I YET chih tinjih nyuh kluị eh njit 1 vih tintiya lye nikhut triniditi ha, E 3

81 
nikha kwuttohndai Vittukoochanchyo vih kuttukootatshe kit, rsikotitinyoo tegwichöei Nikhut triniditi kwizit? Lyet vih kukukwạthut, et vih kutanduk, et vettitinịzya, et yinjitaä, et vih kutunantya eltsik tai ako rsyehkwundai taị; ako chizi tutthug akheochingyoo attun rzị vuh tsuh uttikuttunantya, tạthoog ehthle tsut kwutohndai?

T Trig akọtititenja :

Kwikit titihsiya.

वा Alootai gịhyi akọtititenja :

CHOOTYIN tte chih Trig chih Tinjih tsut $\bigcup$ nuttlatshi nikhut kuttunetitya kenjit?

I Akọtai kooh kinjik akọtukoonchyo ninuttlu guttutetuä.

Gikhyi trig tih ttyi, ko tuh lug, nili ttsut oonji tinjih akotathud nandeitsei nili ha trig nandeitsei niti ha oonji, ako yih kit teigutekhyah, akotukoonchyo :

SIH $M$. nun $N$. nyooh nihnji suh ut eh njit Nikhut triniditi ha, nyih tihsiya ako chinihtelhya choog ttrin kwu ttsut, kwiyendo tsut kwiterzya rshọ, kwiyendo tsut kwitersya rshọ, kwiyendo tsut kwichile rshọ, kwiyendo tsut nersigwichachyo rsho, dhantsik taị, ako kwịrzị kwindai taị, nyet titinihshya, nyeinjit titiha, kwitshi nikhethlut nuwhoh chilchi kwu tsut Vittukoochanchyo kuttukootatshi kit: akọ ei kwu tsut sih kheniteikwichituei nih ntluchihei. 
RSIKOTITINYOO HA TUGWATIN.

ๆ Akọtai thlununulugo-tutunetü̈; ako trig nandeitsei tuh nli ha Tinjih nandeitsei tuh nli ha oonji, attun tte gikhyi kit teigutekhyah.

QIH $\boldsymbol{N}$. nun $M$. nyooh nihnji suh kkui eh njit nikhut triniditi ha, nih tihsiya ako chinitelhya choog ttrin kwu ttsut, kwiyendo tsut kwiterzya rshọ, kwiyendo tsut kwitersya rshọ, kwiyendo tsut kwichile rshọ, kwiyendo tsut nersigwichachyo rshọ, dhantsik taị, kwịrzị kwindai taị, nyet titinihshya, nyeinjit titiha, akọ nih kukwutelhthut, kwitshị nikhethlut nuwhoh chilchị kwu tsut Vittukoochanchyo vih kuttukootatshi kit; akọ ei kwu tsut sih khenitteikwichituei nih ntluchihei.

1 Akotai chun thlununulugo tutunetuä; ako Tinjih niletthug Trig nuttlutune $\ddot{a}$, etunetle kug niyitunea kwunindai ha gikhyi tsut. Ako gikhyi niletthug nitiniei, tinjih nittluitune ä, Trig tletsei njikhyi niletthug tsei kwiniyitutuneä keh njit. Alko tinjih niletthug zut ottunetun, ako gikhyi kayoohniltyin, akotititenja :

CHIH niletthug ka nyooh nihnji nikhut $U$ triniditi ha, sih zyin ha yinji nih chidhelhe, ako tutthug suh kwunttette nih ntlugwihthli: Kwittyi, akọ kwitinji, akọ Chunkyo Rsotitinyoo koh rzi zit. Amen.

Ф Akotai Tinjih Trig tletsei njikhyi niletthug tsei akheotitenja, ako ehthle tsut nitshikwutguttä̈: ako gikhyi akọtititenja : 


\section{Zyunguttochya.}

\section{VITTUKOOCHANCHYO sheggu tsut ịli, tinjih tutthug zyundhantseị, akọ kooh} kunnyantyi, kwu tsuh trigwinji kwunkyo ttsun kwuntlunchịe, sheggu kwundai kinitiyin: chih nyih ultsunnut nih kwịrzi tigwinidhun kooh nittluchịei, chih tinji ako chih trig, nyooh rzi ha kwịrzi kooh tsut tunyinitudhun: nittso Isaac Rebeka ha kwịrzi tsut nikha kookwetundai, kwikit tukoonchyo chih nekunnut thlih ha akọtugitetiya akọ kookugutunatya ei kinjih nugwichinttai, akọ ei kheniteikwichituei kookyidetug kwelttsei, (ei kwu tsut chih niletthug kwuntlunetuei ako othinjik koogooteyin akọ kwitekit koọlị nilị) akọ sheggu kwintluth nikhut tigitinetizhya, akọ ttsui ettun ha tigitechya, akọ nih tugwitetuggun kwikit kookwutendai ; Tesus Christ nuwheh Kukwadhud kirre. Amen.

- Akọtai gikhyi nandeitsei kooh nili ninuttlututandul, ako akotititenja :

Einut Vittukoochanchyo nikhut yih natti tinjih nittsunukoovochya rshọ.

\section{Akọtai gikhyi tinjih tsuh guttekhyah :}

TVUGGUH yoo $M$. ako $N$. thluginazhin rsikotitinyoo nikhut triniditi ha, akọ kwikit kukookwanduk Vittukoochanchyo ndah, akọ chih chunzyunnithligutazi kooh ndah, ako ei kwu tsut kheniteikwichituei koonittlugwituei akọ kwitekit koogweltseị, akọ 84 
kwikit kookwanduk niletthug kwuntlukwutuei akọ otinji, ako kwunli nittsitritanji : Akọ tuchihnyoo kwukkuị ako kwuut nikhut gittetiya, Kwittyi, Kwitinji, akọ Chunkya Rsotitinyoo koh rzi zit. Amen.

T Ako gikhyi chih \%wirzi tigwinidhun nettukootutteä.

VITTUKOOCHANCHYO Kwittyi, Vittukoochanchyo Kwitinji, Vittukoochanchyo Chunkyọ Rsotitinyoo kwịrzi nuwhoh tsuh tivitiyin, akọ nuwhoh kunyootyi, akọ nuwhoh kuvanduk: Kukwadhud rsinichi ha nuwhoh nyooyin vih zyooggutdichi ha; akọ Chunkyọ ttsun kwịrzi tigwinidhun, ako kwu tsuh trigwinji nuwhoh zit tutinyooë chih kwundai zit, kwikit nikha kwuttohwhundai, nunh kug nukootutattha sheg kwundai kuttohwhoya. Amen.

T Akotai gikhyi Kukwadhud vikugguchia kwu tsuh tuheih, chih etunetle choh ehilig tititenja, ko chittitelya.

Tutthug ssozyungili. Chilig. cxxviii.

SSOZYUNGI়LI einut tutthug Kukwạdhud $N$ unachud : akọ tih netende kit utta.

Kwugguh yoo nyuh nli koo koottrid chuttịä: O kwịrzị tsut ịlị, akọ sso tịlya.

Nyuh ut chug choh tuchun kwịrzị nasi kit telya: nyih zze kwuggou nasi.

Nih kkyinut ttle thohchun kit: nih vikugguchiä nugooë. 
Kwunohyin, kwikit sso telya ei tinjih: Kukwadhud unachud.

Kukwadhud Zion kwu ttsut kwikit sso nyih titelya : Jerusalem kwịrzị kooeị kwuttu. nạya tạthoog kwutindai;

Ạa nih kkyinut kooh kkyinut zyunkoohtunạya : ako ttsin-ettun Israel tet.

Kwittyi, ako Kwitinji tsut: akọ Chunkyo Rsotitinyoo tsut kwichintsi kolị.

Nittsọ trootshid kwịlịh, chooggoo koọli, akọ sheggu kwitelya. nunh kwilikwutetha. Amen.

\section{T Ko chih Chilig.}

Vittukoochanchyo nersinigichachyo tunnuwhohchinyoo. Chilig. Ixvii.

\section{ITTUKOOCHANCHYO nersingicha-
chyo tunnuwhohchịnyoo, akọ kwịrzị tsut} akọtunnuwhạin: akọ nyih nyen kwu anttri kunnuwhoh nyantye, akọ nersingichachyo tunnuwhoh chịnyoo.

Nyih netende nunh kuggu kakwutetundai kenjit: nih rsyehkoọlị kwu chassi tutthug kutchinnut tet.

Tinjihnut tinyigitichöe, O Vittukoochanchyo : aä, tinjih tutthug tinyihgitichoë.

O tinjih-kutchinnut ssozy unkoli, ako ttseyin zyunkoli : kwugguh yoo tshoogoochöe tsut tinjih untoututanttri, ako nunh kug kutchinnut tsuh Kukwutadhud.

Tinjihnut tinyihgittichöe, O Vittukoochanchyo: aä, tinjih tutthug tinyihgittichöe.

Akọtaị nunh koo kwunasye kwịrzị kwuttunasya: akọ Vittukoochanchyo, nuwhun thun 86 
khe nuwhoh Vittukoochanchyo, kih kwịrzị tigwinidhun nuwhoh ntlutitelya.

Vittukoochanchyo kwịrzi nuwhoh ntlukootitelya: akọ tutthug nunh kug kwi ttsị gihyuntunachut.

Kwichintsi kolị Kwittyi tsut, akọ Kwitinji tsut: akọ Chunkyo Rsotitinyoo tsut ;

Nittsọ trootshid kwịlịh, chonggoo koọlị, ako sheggu kwitelya: nunh $\mathrm{kwilyetkwutedha.}$ Amen.

I Chilig kwilikooshid, ako Tinjih Trig ha Kukwadhud vikugguchiä ndah nitshikwutgäe, gikhyi vikugguchiä tsuh näe, akọ kooh tsuh uttsetutunetuä, akotititenja :

Kukwạdhud nersingichachyo tunnuwhoh chịnyoo.

Kwiti trigikhyi. Christ nersingichachyo tunnuwhoh chịnyoo.

Gikhyi. Kukwạdhud nersingichachyo tunnuwhoh chịnyoo.

NUWHOH Ttyi zyeh zit nih kwilnjik. Nyooh rzi chootsi. Nih kookukwadhud nuvizi. Tinịdhun nunh kuggu tugoochyo, nittso zyeh zit troogwatsi kwikit. Chih ttrin zit nuwhun enyantsit ttrin ndo thloogootunazya nuwhoh ssih. Ako nuwhot troogwandyoh nuwheh unkoochịli nittso unkoochitili einut troogwandyoh nuwheh tungittiyin. Kookutkwutundai tsuh nuwhoh tunọe rshọ: ko troogwandyoh kwu ttsut nuwhoh yunninji. Kwugguh yoo nih ttsun 
nilị kookukwadhud, ttuih, akọ nindelh sheggu keh njit akọ sheggu keh njit. Amen.

Gikhyi. O Kukwadhud tinjih nyuh kanduk, akọ trig nyuh kanduk kooh nyạssi.

Kwiti kwi kinjik. Einut nyih tugihchanchyo.

Gikhyi. O Kukwạdhud, nih tekit rsikotitinyoo $\mathrm{kwu}$ ttsut $\mathrm{kwu}$ tsuh trigwinji kooh tsuh tọë.

Kwiti kwi kinjik. Akọ sheggu kooh kunyantyi.

Gikhyi. Zze yettug kwịë nugwichinttai kooh tsut oli.

Kwiti kwi kinjik. Truttukoochachyo nih nyun kwu ttsut.

Gikhyi. O Kukwạdhud nuwhoh gichinchi odhontshe.

Kwiti kwi kinjik. Akọ nuwhoh kwurzrulh nyeh nuchootshit.

\section{Gikhyi.}

\section{VITTUKOOCHANCHYO Abraham} tsut, Vittukoochanchyo Isaac tsut, Vittukoochanchyo Jacob tsut, chih nih ulttsunnut kwịrzị tukoohnyayin, akọ kooh ttri zit sheggu kwundai nittshi nọlya, chitti tte nih Itunetle Rsotitinyoo zit tsenja tsut kikyigutandul, thlih ha kwikit tugitettiya. O Kukwạdhud zyeh kug kwu ttsut zyooggutdichi ha kooh nọyin, ako kwịrzi tsut tukoyin. Akọ nittsọ Abraham, Sarah ha nih kwịzi tigwinidhun kooh tsut nukoochidhae, kwintluth kwu khu88 
gwizhit kwu tsut, kwikit tukoonchyo chih nih ulttsunnut nih kwirzi tigwinidhun kooh kkọ nukoochạë: nih yinji kookukwathut, akọ sheggu ettersyeh gili nih kookunatyi kirre, nih chettigwinidhun zit tigitechya tathoog kookwutendai kwu tsut; Jesus Christ nuwheh Kukwadhud kirre. Amen.

\section{ๆ Chih gichinchi kendo akheötitetinja, nichin tai Trig kiyendo trizhit tai.}

KUKWADDHUD nyeh rsikoonchyo, akọ Kwittyi zyeh kug, nih ozi zyooggutdichi ha tinjihnut lyei zyungilye: Nih tsut zyungittinichi kwịzi tigwinidhun ha chih nekunnut kooh tsuh trininji, ehthle tsut kwirzi gitelya kwikkyinut gitettiya, ako kwikit nizhook nikha kookwutetundai Vittukoochanchyo tsut chettigwinidhun ha ako rsittuttsut kooh kkyinut tikyinchik kuttunaya Christ netinde kit ako tshoogoochoë tsut, nih tugwitichile ako nih yinjikwichile kwu tsut; Jesus Christ nuwheh Kukwadhud kirre. Amen.

VITTUKOOCHANCHYO, nun nyuh
ttuih nugwichinttai kirre tutthuge koochichyo kwudhantsei, elyet ka kwilị ttsut ; ako tte (chizi ekoochichyo kirsyenukwaei kootle), kuttukoochidhantshei, tinjih ttsut (nih kit elttsi ulttseị) trig kwịlịh ; akọ nikhut koohnidhantye kirre kunnuwhoh nyidhantyin elyet tte tshoogootutteä nittseguttetaul einut nikhut triniditi ha ihthloog kooh dhantsei: o Vittukoochanchyo nikhut triniditi kooei rsikotitinyoo kwudhantsei, akọtinchyo nunaị 89 
kwichinttsi kwu tsut; kwi zit kwikirre akọtigwinyoo tigwinyoo, ako kookukwunatye kwunkyọ kit nikhut triniditi, akọ nikhut ginithityi Christ vih thlachyakthut ha ekyidettug; Chih nyih ultsunnut chehrsịkoonchyo ha kooh nyayin, chih tinjih tih trenjo ggut titinezhya, nih kinjik kit (nittso Christ tih trenjo, ei yoo thlachyakthut, ako eh njit kwuntlututachị, ggut tinizhin akọ zyooggutdichi ha kuniltyị, attun thun vuh tthui kit tinchyo) akọ chih trenjo thui tuh kkuị ggut titinezhya, ako rsikoochanchyo ha akọtiyihtaya, akọ thlië tsut yih kukwutathut; âkọ tutthug kettritsoogoochichyo ha, akọ khetachyo, ttsinettun ha, trenjo rsotitinyookthut ako Vittukoochanchyo kit titettiya. O Kukwạdhud ehthle tsut kwịrzi tukọyin, akọ tukoohtọnyoo nih kukwadhud sheggu tsut kugutettiya; Jesus Christ nuwheh Kukwadhud kirre. Amen.

\section{ๆ Azyuk kootle gikhyi akotititenja:}

THLACHI ha nichinttai Vittukoochanchyo 1 nunh koogwinyathli tai trootshid nuwhoh yuggunkthut Adam Eve ha eltseị akọ rsotitinyoo eltsei, ako nikhut kooh nilti nikhut triniditi ha; vih chehrsikoonchyo kwichile nuwhoh kko nechoonja, rsotitinyoo nuwhoh tsya, ako kwịrzị tsut tunuwhohyin, sso vuh tsun tohtsya keh njit kwizyin kwunkyo ha, ako nikha kwutohwhundai chettigwinidhun rsikotitinyoo ha nuwhoh kwundai kwilikooshid kwil tsut. Amen. 
- Ei the kwigikhyi kooli kkwa chi tinjih trig ha koo koottrid kookukwunatye tsut, gikhyi kwikit teiguttekhya.

NUWHOT tutthug nikhut tunnohtshei, ko IVo tunyinoohthun nikhut triniditi rsikotitinyoo ha nikhut tutunnohtsha, zyunodhudhohtshei chitti tte Kwuttunututluth Rsotitinyoo tenyoo, kwukkui, kooh koottrid kooh ut tsut, ako kwuiut kooh kkui tsut.

Paul rsotitinyoo, tuh etunetle zit Ephesus tinjih tsut, ihthlokwunli kookuäzye zit, chih tugwitịe tinjih tutthug gechiltshei kthut tsut kwuntlukwituei : Kwukkui nuwhoh ut kwet tunyinoohthun, nittso Christ thlachyakthut ggut tinizhin, ako eh njit kwuntlututachi, rsikotitinyoo kwuttatsya ako kitata choo ha kunutattrha, kinji kirre; uttuntlukwutetuä thlachya nindelh ha gelyẹ, elyet koogwichithuggut ha ettun, ssyo ettun, ko ekoochichyo kit tinchyo koo kkwa: ko rsikotitinyoo zyungitelya, ako koogwichinji kettun. Kwikit tukoonchyo tinjih kooh ut ggut tungittinezhya khe kooh zyin kwikit. Ei tuh trenjo ggut trinidhun uttinutudhun: kwugguh yoo tthui tte elyet ihthle tuh tthuị tuttroutilchyo, ko kwịrzị kunatyi akọ chettigwiniahun ha kunatyi, nittso Kukwadhud thlachya ggut tinizhin: kwugguh yoo kookwiyerzra zyunitilị vih zyin ttsut, vuh tthui ttsut, ako vuh tthun ttsut. Ei keh njit tinjih tih ttyi tuh hun ha akheötitenja, akọ tuh ut ettinetya: akọ ei nekunnut ihthloog tthui gitettiya. Chih yoo kwintluth nunaị: ko Christ thlachya ha eh njit ginihkhyi. Nukoolyo, nuwhot tekookwi- 
nyachyo tuh trenjo ggut tinyoozhin, nittso uttinutudhun.

Chun ei Paul rsotitinyoo, Colosse tinjih tsut uttuntluth, tinjih gichiltsheikthut tutthug, kwikit tsuh gịkhyi : Kwukkuị nuwhoh ut kwet tunyinoohthun, ako kooh tsut trinohtsye rshọ.

Chitti Peter rsotitinyoo Christ chilhe tenyoo thui zyunodhudhohtshei, ei attun thun kidhiti ịlịh ei gichiltsheịkthut tsut akọtinyoo: Nuwhun Kwukkui, nuwhoh ut ha tunohchyo kakwutundai $\mathrm{kwi}$ kit; kwu tsuh yinjikwilchile kooh nittlutohthli, ttyạ uttrekul kit vuh tsut, ako kwundai kwi kwu tsuh trigwinji nikha ku whohyin gelyẹ, nuwhoh gichinchi elyet zukukgotutulha.

Tzet kwu tsut zyunkwutohtthuk tinjih ettelye kwikit titettiya tuh trenjo tsut. Chooggoo tthui, nuwhun kwuüt: Zyunodhudhohtshei, ako kikyunnohnji nittso nuwhoh kkuị tsut kwikit tuntowhohya, nittso Kwuttunututluth rsotitiryoo zit kwirzi kookwunatye.

Paul rsotitinyoo ei Etunetle Ephesus tinjih tsut kwiyetshi veh getye, kwikit kunnuwhoh natun: Kwüit khe nuwhoh kkuị kooh tsut chunnetotowhohnyoo rshọ, Kukwadhud tsut kwikit. Kwugguh yoo kwukkui trig tshid nili ; ako nittso Christ thlachya tsut tshid nilị, akọ kwizyin kwu Assi nili.. Azyuk kwuggut nittso thlachyakthut Christ nettshiggutunazik, kwikit tukoonchyo kwüut khe kuttu kkuị kwikit zyunkolị tutthug ekoochichyo ha. Ako chun akotinyoo, kwuut kwinyooyin tuh kkui yinjitaä.

92 
Akọ tih etunetle zit Colosse tinjih tsut, Paul rsotitinyoo chih kaönitun nuwhoh ntlugwitutuei: Kwuut khe nuwhoh kkui tsut chunnetotowhohnyoo rshọ, nittsọ Kukwadhud tsut tshoogoochöe nili.

Peter rsotitinyoo tthui kwintluth kwịzi tsut kunnuwhoh natun, akotinyoo; Nuwhun kwuut, nuwhoh kkui nuntshitunnohzik; chettenut kinji kunkookwelhthut chi, Kinji ettun thui kwuut kooh nutinde kirre khunkookwutazya; kạthoog nuwhoh nutinde khetachyo nuchut ha, zyunkoogoonayin. Einut nunkookwazi, ei kwuüth kwunukwazi tshihnatlya, yootsei dhuttho nungilizye, nunegilizye; ko kwittri zit tinjih nunaị koli, ei yoo. elyet kwuttuthutrurh; ei yoo vahtrinititloo kwunkyo kettritsoogoochichyo akọ khetachyo, Vittukoochanchyo ndah kwintluth ginili. Kwugguh yoo ako tukoonchyo tenotaị trenjo rsotitinyookthut, Vittukoochanchyo tunguttanchyo khe zyunginithitli, kuttu kkui nuntshihguttunazik ịlịh; nittsọ Sarah Abraham kukwelthut, Kukwadhud yah nyoo; vih yetsinut zyunohthli akọ tạthoog kwịrzị tunwhohyin, akọ elyet zyunnudhohchut kwuttakthou ha.

9 Tinjikwitizhit nikhut niditti kechid tlukoovanji rsotitinyoo koogootendul, nikhut giniditi tai, ko trootshid kwikit nukoochazhi nikhut giniditi tle. 


\section{BURIAL OF THE DEAD.}

\section{NITTSO NININCHIKKTHUT KOOHNUNTANDEI.}

T Kunukoondei einut kwunneinittunja, ko nettigichachik elyet kweh njit chih gichinchih tugwichili.

9 Gikhyi tthunkit kwu tsut nyakwuth etshi tuttutehah, ako kwiyetshi tehah, gichinchih zze kwu tsut, ko tthunkit kwu tsut, ako alotititenja :

NUNUKWALYE kwundai ha ihthli, Ku1 kwadhud nyoo; ei sih kyinjizhit, ninidhud kọ, kwuttuzuk kwutendai : akọ chootyin tte kwundai ako sih kyinjizhit, elyet tte nutethah. St. John xi. 25, 26.

Suh Assi kwundai kahsundai, ako huịko taị nunh kuggu nutedhut. Akọ suh dhoh tle chih kwizyin kyoo rsyetekunyitutathle, kwuttuzuk suh thui zit Vittukocchanchyo tunelhya: khe njit tunelhya, ako suh nde yih tunaya, ako chizi ukkwa. Job xix. 25, 26, 27.

Chih nunh kug ekoochichyo nugwinitilye kkwa, ako ettelye koogootechin ekoochichyo shọ kwichitili. Kukwadhud kwuntlukwantshị, akọ Kukwadhud neyoohnjik: Kukwadhud vooh rzi tetchoottsi. $1 \mathrm{Tim}$. vi. 7; Job i. 21. 
NITTSO NININCHIKKTHUT KOOHNUNTANDEI.

\section{Gichinchi zze kwi zit nitte neginchya tai, chih chilig kwititetitsha:}

\section{CHILIG XC.}

KUKWADHUD, nuwhoh kwu tsuh kulI chichya tekit ịlih: ihthloog nizyoọndichi $\mathrm{kwu}$ ttsut attun kwu tsut.

2 Ddha kwelttsei kwiyetshi, akọ nunh akọ nunh kug kwelttseị kwiyetshi : sheggu kwu ttsut Vittukoochanchyo ịlí, akọ nunh kwilikwutetha kwu tsut.

3 Tinjih rsyetehkoogoochạthli : chun akọtuchịnyoo, tinjih kwi kkyinut kwẹe nunohchya.

4 Kwugguh yoo ihthloogchotyin tinili kit chotyin tsut chotyin nugwutudhut nyih nda ketuị kit tukoonchyo: ttodh zit khuötayin kit kwuto kwitizhit.

5 Inchit tsut rsyetetkoochilye, gilchyuth kit tunginchyo : akọ inchit tsut ttlo kọt zyunkuttututrurh.

6 Vvun anttlo ako nasye: kọ khah tsut khuttiti, tizyeih, akọ tuttutrurh.

7 Kwugguh yoo elyet sso enili uttitidichye: akọ zyunnachut nyih vecho gelyẹ.

8 Nuwhot troogwandyoh nyih nda nukwinilye: ako nukwuzuk nuwhot troogwandyoh nih nyun attri nda.

$9 \mathrm{~K}$ wugguh yoo onyacho taị, tutthug nuwhot ttrin zit kwuto kwititizhit : nuwhoh nukwutudhut kwilukooshit nukwitinitili kwunduk keh kwanduk kwi kit.

10 Nuwhot ttrin zit nuntshitutthui chitsuëtsinekthuị tsut chotyin nukwotudhut kwe- 
tilị ; akọ tinjih kwikit nungichinttai nikitankthut tsut chotyin nukwutudhut niginchya: kwuttuzuk kooh thuih koottridtugwayin ako troogwiti ha koọi kwirzi ; kwikit anidhook kwititizhit, ako kethle tichidichye.

11 Ko chootyin tte nyih vecho Kwuttuih nayin : kwugguh yoo ei kwikit nittso tinjih nachut kwikit tukoonchyo nyih vecho koọi.

12 Nuwhot ttrin zit zyunk wititititsha kunnuwhoh nyạtun: nuwhot ttri kwizye kwu tsut kwuntluyinjitititilya.

13 Kukwạdhud koochid chun uttsunettinyae: ako nih ultsunnut tsut zyoogguttinidichyo.

14 Nih rsinichi ha rsyunnuwhoh tinyadhun, ako nyakwuth kwu tsut: akotukoonchyo sso zyuntitilya, ako tseyin zyuntitilya tutthug nuwhoh kwundai kwi ttrin zit.

15 Chooggoo khunnuwhanchi khuịnjinuwhoh udhạnchik kwu tsut kạthoog kwikit: akọ ei nukwutudhut eh njit khuinjidhidichik.

16 Nih ultsunnut nih koottrid kookunkoohnyatye : ako kooh kkyinut nyih nindelh.

17 Ako Kukwadhud nuwhoh Vittukoochanchyo vih nindelh chịttsi nuwhoh kkọ kolị: nuwhoh nli koottrid nuwhoh kkọ ndokootanta; nuwhoh nli koottrid ndokootanta.

Kwittyi akọ Kwitinji: akọ Chunkọ Rsotitinyoo ha chịnttsi kolị ;

Nittso koogwinyathud kwịlịh, chooggoo koọli, akọ sheggu keh njit kwitelya: nunh kwilikwutetha kwu tsut. Amen.

\footnotetext{
I Azyuk kootle Paul rsotitinyoo uttunututluth trootshid Corinth kwi tinjih tsut, ihthloog96
} 
KOOHNUNTANDEI.

chotyin tsuh ihthlothkwunli vkiuäzye ttsut lewititetitsha.

1 Cor. xv. 20.

NHOOGGOO yoo Christ etshị kwu ttsut ninelikhyin, ako einut ganchyoth tet trootshid tinitirzi dhitlit. $21 \mathrm{~K}$ wugguh yoo nittso tinjih kirre etshi nukoochatthut: kwikit tinjih kirre thui etshi $\mathrm{kwu}$ ttsut ninukwalye nukoochatthut. $22 \mathrm{Kwugguh}$ yoo nittso tutthug Adam kirre nigininchik kwikit tukoonchyo thui Christ kirre tutthug zyunkookwutendai zyunkootattsya. $23 \mathrm{Kọ}$ ettounyachyo ehthlooggoo kwitili. Christ trootshid tinitirzi, kootle einut Christ ttsun zyungịi kunutetik taị. 2t Akọtaị huịkọ nukootutattha, Vittukoochanchyo $\dot{K}$ wittyi tsut kookukwadhud kwuntlukwititetua: tutthug kukwadhud akọ tutthug kuttukootadhud, ako kwuttuih rsyetehkoogootutathlye taị. $25 \mathrm{~K}$ wugguh yoo ette lye kukwutadhud tutthug kwutsuhkwilheikhthut tuh kthui zug nutelya kwu tsut. $26 \mathrm{Ei}$ huịko kwutsuhkwilheị rsyetehkuttutalye etshị nilị. $27 \mathrm{~K}$ wugguh yoo tutthug ekoochichyo tuh kthui zug nukwitinilye; kọ akọtinyoo tai, tutthug ekoochichyo vuh zug nukoochazi, ette lyẹ kwirzye koọli, elyet kwititithitshi, ei yoo tutthug ekoochichyo vuh zug nuk witinilye. 28 Ako tutthug ekoochichyo vuh tsut kwitugtekwitittechya tai, akọtaị K witinji tthui yih zug telya, ei yoo tutthug ekoochichyo yih zug nukwititelya, Vittukoochanchyo tutthug zit tutthug telya gelyẹ. 29 Nithlinetsi chi ttungwittiya einut nininchikkthut eh njit kwunneinyithunja, thlạchi ha nininchikkthut elyet 
nunnelkye chi; chyaggut tte thui nininchikkthat eh njit kwunneinyithunja? 30 Ako chyaggut tte ettettukoonyachyo kookochitri koukonyoo nunchidhazye? 31 Nuwhoh sso kooli kirre Jesus Christ nuwheh Kukwadhud kirre kihsiyin akotechihnyoo ttrin kootetkwinyachyo ninihshit. 32 Tinjih kit ginihlshyi utthuị ndyoh niitsilhthut chi Ephesus zit, chitti tte seh tsut kookoolị, nininchikkthut elyet nunnelhilye chi ? zyunoä akọ zyunwhonyin kwugguh yoo nika ttrin zit ninidichi. 33 Zyunnunuwhoh nyootue rshọ; kwịrzị kkwa nikha trigịkhyi kwịrzi kwinutinde tutugwitashit. 34 Tshoogoochoe kwu tsut kunnohnji; ake troogwandyoh tunwhohyin rshọ: kwugguh yoo chettenut elyet Vittukoochanchyo hazyungithinjik, nuwhoh ozye kwu tsut akotechihnyoo. $35 \mathrm{Ko}$ ihthle akọtititenja : nittso tte nininchikkthut nuttutata? ako chitti kwizyin tte ha neginchya? 36 Troogwinjik ịlị, ei ninili elyet kilhzhi tunachud chi kwirzị. 37 A ko ei ninili elyet ei kwizyin nidhịli ei yoo kwittelya, ko nitshi kwirzi, ttloli rshọ ko chizi kwuntshi kookwitelya rshọ. 38 Kọ Vittukoochanchyo kwizyin kwuntlutuei nittso kettinizhin, ako ettetkookwinyachyo kwuntshi khe kwi zyin. 39 Tutthug kwutthui elyet ehthloognchi kwutthuị kwili, ko ehthloog kwutthuị tinjih ttsun koọlị, chizi utthuị utthuị ttsun koọli, uttun thlooggoo ttsun koọli, akọ chizi nyin ttsun koolị. 40 Zyeh kug kwizyin thui zyunkoolị, ako nunh kug kwizyin : ko zyeh kug kwizyin kwi nindelh ihthloog kwitili, akọ nunh kug ttsun ihthloog kwitili. 41 ksye nindelh ihthloog koọlị, akọ ttodhorzi 98 
nindelh ihthloog koọli, akọ ssun kwi nindelh ihthloog koọi : kwugguh yoo ssun ihthloog chizi ssun netsi tinchyo nindelh ba. $42 \mathrm{~K}$ wikit tukoonchyo tthui nininchikkthut nunnukootalye kwitelya : kwizyin tetizhit ha nazi, elyet tetilhzhit ha ninukwichachye. 43 Elyet yinjikwichae ha nazi, nindelh ha nichachye: Nitrugoochintlook ha nazi, nugwichinttai ha ninukwichachye : 44 Utthuị kwizyin ha nazi, kwunkyo kwizyin ha ninukwichachye. Utthui kwizyin koọli, akọ kwunkyo kwizyin ha koọli. $45 \mathrm{~K}$ wikit tukoonchyo tthui attunututluth: Trootshid tinjih, Adam, kwunkyo kwundai elttsei huịko Adam kwunkyo kookwundai kwuntlutuei elttseị. 46 Kwuttuzuk elyet ei kwunkyo kookwundai trootshid kwilị: kọ ei utthuị, ako tle ei kwunkyo kookwundai. 47 Trootshid tinjih nunh kwu ttsut, nunh kug ttsun nilị: ako nikendo tinjih, ei yoo Kukwadhud zyeh kug kwu ttsut. 48 Nittso ei nunh kug kwu ttsut nili kwikit tukoonchyo tthui einut nunh kug ttsut zyungịli ; ako nittso ei zyeh kug kwu ttsut nili kwikit tukoonchyo thui einut zyeh kug kwu ttsut zyungili. 49 Ako nittso nunh kug ttsut koọi vih kit telttsị tinitilye, kwikit tukoonchyo tthui zyeh kug ttsut koọli vih kit telttsị zyuntititilya. 50 Ako chih yoo techihnyoo, kwuchahnut Kwutthuị, akọ kwutta elyet kookwidhun zyeh kug kookukwadhud kookwitelya; ako tetizhit tetilhzhit elyet kithii thui. $51 \mathrm{~K}$ wunohyin nukwinai akotunnuwhakhnyoo: Elyet tutthug tudhachyuth, ko tutthug etchooggoo tuntitinja: 52 Inchit tsut, netotruttutazhi kạthe, huịko 
yoozyoo taị; kwugguh yoo yoozyoo tudhatshik, akọ nininchikkthut nunttalye elyet kookwidhun tetichye, akọ etchoog tuntitinja. $53 \mathrm{Kwugguh}$ yoo chih kwizyin tetizhit ettelye elyet tetilhzhit nutandul, ako chih kwizyin kwinizhit ettelye elyet noshiddi nutandul. 54. Ako chih kwizyin tetizhit elyet tetilhzhit nutandul taị, akọ chih kwizyin kwinizhit elyet noshid nutandul taị, akọtạ ei kinji kwuttunututluth akotugwitanja: Etshi kwutugtigwichtchyo zit kwutanji. 55 Etshị nichin kwaun tte nih kwichitsik? Tthunkit nichin kwuan tte nih kwutugtigwichichyo? 56 Etshị kwichitsik eị yoo troogwandyoh koọị, ako troogwandyoh kwu ttuih ei yoo tugwitịe koọlị. $\quad 57$ Kọ Vittukoochanchyo tsut hụ kolị, ei yoo kwutugtigwichichyo nuwhoh ntluchịli nuwheh Kukwadhud Jesus Christ kirre. 58 Azyuk kwuggut, seh chahkthut nuwhet tinutudhun kwiyeinjohchi, nunkutowhohta rshọ, sheggu Vittukoochanchyo koottrid ha koottridtungohyin, kwugguh yoo kazyunwhuhndai elyet nuwhoh koottrid Vittukoochanchyo tsut zyọ kwilị.

\section{Tthunkit kwu tsuh neginchya tai, tạthoog etshittya tthunkit kwi zit vih rsyenutandul, gikhyi akotititenja :}

TINJIH trenjoh ttsut vuh kooli nyakwuth I rzị kwu tsut kwundai, akọ khuịnjikwutudhut ha khuịjitudhut. Tikyizhit ako chuttun kit huttritti; kwunkyo kit untitizhit, ako ehthlooggoo tilị kkwa.

Kookwundai kwinjirh, etshị zit zyunitilị: chootyin ttsut tte voọkuntinititya nuwhoh 100 
tsuh trutendul keh njit, ko nun ttsut Kukwadhud, nuwhot troogwandyoh keh njit tshitchöe tsut elyet sso enili? ?

Kwuttuzuk, O Kukwạdhud Vittukoochanchyo kwiyendo tsut rsochitinyoo, $\mathrm{O}$ Kukwädhud kwiyendo tsut nichinttai ịli, Rsochịtinyoo, ako Assi kwiyendo tsut nyeh rsikoonchyo, etshị sheggu tsut kwurzra khuịnjikwutudhut kwu tsut nuwhoh ntlutọlya rshọ.

Kukwạdhud nuwhoh ttri ttsun nukwinai kanyitundai; nuwhoh gichinchi tsut nih tzi ketrsikoonchyo tuchöe rshọ: Kọ Kukwạdhud kwiyendo tsut rsochịtinyoo nersingichachyo tunnuwhoh chịnyoo: O Vittukoochanchyo kwiyendo tsut nichinttai, rsochitinyoo ako Assi nyeh rsikoonchyo, kuttoutichatri kwiyendo tsut ginili sheggu tsut ili, etshi $\mathrm{kwi}$ khuịnjikwutudhut kirre huịko tsut etitsye tunnuwhọzhin rshọ.

I Ako tathoog nunh etshittya kug kwu tsut tazi ihthle kerhe nudhut kirre gikhyi akotititenja:

K WUGGUH yoo Vittukoochanchyo thlachi I ha nichinttai sso ilịh vih chersikoonchyo kirre, nuwhoh chah ninidhud nikyo ochithinjik, azyuk kwuggut vih zyin nunh zit nunnachye: nunh nunh tsut, thlyeth thlyeth tsut, ako thlitsi thlitsi tsut; sheggu kwundai kwu tsut ninukwalye thlih ha kunkovitili nuwheh Kukwadhud Jesus Christ kirre; ei nuwhoh zyin trandyoh etchooggoo titelya, vih zyin chinttsi kit titelya keh njit, vih koottrid nichinttai kirre nittso kudhun tutthug ekoochichyo uttut tsut kotetundul. 


\section{बा Akọ tle akọtititetinja:}

YYH kug kwu ttsun kootezyoo teihtthuk, $\triangle$ akotusahnnyoo: Ettinintluth: Chooggoo kwu ttsut sso zyungịi ei nininchikkthut Kukwadhud zit nigininchik: Unkyo akotinyoo; kwugguh yoo kooh koottrid kwu ttsut zyunnugazi :

ণ Azyuk kootle Gịhyi akotititenja:

Kukwạdhud nersingichachyo tunnuwhoh chịny'oo.

Christ nersingichachyo tunnuwhoh chinyoo. Kukwạdhud nersingichachyo tunnuwhoh chịnyoo.

NUWHOT Tंtyi zyeh zit nih kwilnjik. 1 Nyooh rzi chootsi. Nih kookukwadhud nuvizi. Tinịdhun nunh kug tugoochyo, nittso zyeh zit troogwatsi kwikit. Chih ttrin zit nuwhun enyantsit ttrin ndo thloogootunazya nuwhoh ssih. Ako nuwhot troogwandyoh nuwheh unkoochịli nittso kooh ttsut unkoochitili einut troogwandyoh nuwhet tungittiyin. Ako kookutkwutundai kwu tsut nuwhoh tunöe rshọ: kọ troogwandyoh kwu ttsut nuwhoh yunninji. Amen.

\section{Gikhyi.}

VITTUKOOCHANCHYO thlanchi ha nichinttai tinichyo, nyah zyunkookwundai kwunkyọut einut tzet kwu ttsut kethle kidichya Kukwadhud kyinjigichi ; akọ kwikyinjichinut kwunkyonut, kwutthui, kwu102 
kdha kwu ttsut zyunkotetundul, sso ha ako tseyin ha nyah zyungili : kwittri ha huị nih ntlunchituë, kwugguh yoo sso ịlih chih nuwhoh chah chih nunh kug trandyoh kwi khuinjikwutudhut kwu ttsut ochidhitinjik; ako nih tsut zyungittinichi sso ịli chi nih chehrsikoonchyo kirre nyakwuth nih uttoukonyookthut kootetkookwinyachyo kwitshilikootia ako nih kookukwadhud khuntut nutahah; akotukoonchyo nuwhun ako tutthug einut ha nyooh rzi rsotitinyoo zit thlih ha kwikyinjigwizhit ha chih kwundai kwu ttsut kethle gichochil, zyunyinjikwutunattuih, ako sso zyuntitilya thlanchi ha, kwizyin zit ako kwunkyo zit, sheggu kwu tsut akọ elyet kwilikwutetha nih nindelh zit, nuwheh Kukwadhud Jesus Christ kirre. Amen.

\section{Gichinchi.}

0 VITTUKOOCHANCHYO zyoogguttinidichyo, Kwittyi nuwheh Kukwadhud. Jesus Christ, ei yoo ninukwalye ako kwundai nili ; vih kirre chootyin tte kwikyinjizhit kwutendai, ninidhud ko; ako chootyin tte kwundai ako yih kyinjizhit, elyet sheggu keh njit nutetha; ei vih chileh Paul rsotitinyoo kirre thui kunnuwhoh niltyin elyet zyuntroogwitidhititi, tinjih elyet kunkookothli kit, einut Jesus zit gelchyuth eh njit: chetukotithinyoo ha nih tsut zyungittinichi, Kwittyi, troogwandyoh kwu ttsut etshị ttsut nunnuwhathle tshitgichöe kookwundai kwu tsut; azyuk kwuggut nichin tai chih kwundai kethle titutah, vah zyuntitilya nittso kunko103 
vitili chih nuwhoh chah kwi kit tinchyo: ako tutthug nunukootalye huịko ttrin zit tai, nyih nda kuntitilya zyunnuwhoh tetundulh; ako ei kinji nirzi zyuntoututitundul nih Tinji vet tinutudhun, akotaị tutthug nyet tunginidhun ako nyungunachut kooh tsuh gutekhya, akotititenja: Ukwẹe nunnohchya, Ttịa vih kkyinut ssozyunohthli, nunh koochotli taị kwu ttsut kookukwadhud nuwheh njit rsyenungotli zyunonohnji: Nuwheh njit kwi kit tinịdhun nih tsut zyungittinichi Kwittyi zyooggut - tinidichyo, nuwhoh ekyidettuggikhyi ako nuwhoh Assi Jesus Christ kirre. Amen.

UWHEH Kukwadhud Jesus Christ vih 1 zyooggutdichi, ako Vittukoochanchyo vih chettigwinidhun, ako Chunkyo Rsotitinyoo nichya sheggu nuwhot tutthug nuwhah tungoochyo. Amen. 


\section{H I L I G.}

nnonnon

c.M.

I.

St. Ann's.

1 Ttiạ trootshid tinjih dhantsei

Thlih ha kwịrzi tịnchyo

Kọ kwirzoọ tettiyin kirre

Nersichachyo dhitlit.

2 Tinjih nih kinjik thlechilnei

Troogwandyoh tettiyin

Ei keh njit choog huịnjitudhut

Kidhun uttu tazi.

3 Ko nih chetrsikoonchyo kirre

Nih Tinji kwuntlantshị

Tinjih Assi telya keh njit

Jesus tinjih dlititit.

4 Jesus tinjih tekit koonjik

Nersitichilchyo thlih

Tinjih troogwandyoh kwinịkdhei

Nikyito kug gulttsuk.

5 Kọ Christ tinjih ggut ninidhud

Kwundai khugwitäei

Choog yoo chootyin yih kyinjizhit

Troogwandyoh ttsut tizi.

6 Ttia nih tsut untotili

Huị nih ntlunchitäe

Nih Tinji Christ nuwhoh ntlantshị

Nuwhoh tassi keh njit.

105

F 3 
CHILIG.

L.M.

II.

Old Hundred.

1 Vittukoochanchyo vih Tinji

Kwintluth nuwhet tunyinidhun

Jesus nuwheh njit ninidhud

Troogwandyoh ttsut nuwhoh tassi.

2 Chootyin Jesus Christ kyinjizhit

Vih troogwandyoh ekootichya

Troogwandyoh kwu ttsut tetizi

Akọ zyeh zit kwu tsuh tehah.

3 Jesus nih ttsun zyunitilị

Nih tsut sso ha untotili

Jesus sheg nuwhoh kunnyantyi

Akọ nuwhoh tsuh trunyundai.

4 Tsenja tsut kunnuwhoh nyạtun

Nih chettigwinidhun chile

Thlih ha hazyunitundai chi

IKwintluth tsut ssozyuntitilya.

5 Sheg nyet tunyitinitizhya

Nih kinjik sheg kunkwutata

Kwintluth tsut tunyinjitizhit

Yette zyeh zit nutitutah.

C.M.

III. Cambridge New.

1 Tinjih Dhantsei nyet rsikoonchyo

Nih chettigwinidhun

Nih Itunetle choh nikyo

Kwirzye koogootechin.

106 
2 Tinjih nersichachyonut yoo

Kwizit kungookwäei

Nunh kug kwichilo yendo tsut

Kwichile sheg keh njit.

3 Tzet tinjih Christ yih tsuh gịkyi

Tutthug zyunodhotsha

Sheggu kwundai kwuntlutäei

Chootyin yih kyinjizhit.

4 Christ nih tsut zyungittinichi

Nuwhoh tsuh trunyundai

Nih kinjik zyunotudhatsha

Nih kunkwutata tsut.

4. 6.'s. 2. 8's.

IV.

Lennox.

1 Vittukoochanchyo choh

Vet tunyinitudhun

Chilig vuh tsuh untotil Tutthug nuwhoh ttri ha.

2 Nuwhoh Ttyi zyeh kuggu

Nuwheh tunyinidhun

Nuwheh njit kwintluth chuịnirzi

Nuwhoh tassi keh njit.

3 Katrugwetinjik tai Thlih ha nersintachyo

Tih Tinji Christ nuwhoh ntlantshi

Zyuntitizi keh njit.

4 Choog ssozyunvitili

V:ttukoochanchyo tsut

Zyeh kug kwu tsuh itutah yoo

A yuk sheg tutatsha.

107 
5 Christ vih nyun tunnaya

Nindelh zit tunnaya

Sheggu zyeh zit vah tutatsha

Thlih ha ssokwitelya.

\section{I'm going home.}

1 Zyeh zit sih zze treshit kwịrzi Zut tsik chizhik ha kwitethlya Zyeh zit rsye yendo tsut chattri

Zyeh kug zze seh ttsun kwittelya.

Yettug tihsya, yettug tihsya,

Yettug tihsya, chun telhchya kkwa

Chun telhchya kkwa, chun telhchya kkwa Yettug tishya, chun telhchya kkwa.

2 Ttiạ vih zze yette kooei

Yettuggu ssu yettug nizhit

Chih nunh kug kethle chibseih chi

Zyeh kug zze seh ttsun kwittelya.

Yettug tihsya, yettug tihsya, \&c.

6.6.4.6.6.6.4. VI. National Anthem.

1 Chun chihthlud kwiyetshih

Tzet rsretetridhichil

Untotili.

Jesus nuwhoh Assi

Vih chettigwinidhun

Vih chetrsikoone hyo ha

Thlih ha chinttsi.

2 Tthui vah zyunitundul

Nuwhet tunyinizhin

Thlih ha nirzi

108 
Nittso tuntitiya

Vih cheinirzi keh njit

Sheg vih kunkwutata

Nuwhoh ttri ha.

8.7.

VII.

Fount.

1 Ttia nih tsut gittinihchi

Nersichachyo sochịnyoo

Jesus Christ suh Assi kirre

Kwinthluth nyookkut ginihkhyi.

2 Yette zyeh zit soh dhudhantshei

Suh gichinchi oninji

Chunkyọ nirzị suh ntlunyantshi

Sih tsuh trutendul keh njit.

3 Ttia trootshid set tinịzhin

Nih zyooggutdichi kirre

Nih Tinji Jesus suh ntlantshi

Akọ seh njit ninidhud.

4. Chitti nyeh njit tettihsiya

Nih chetrsikoonchyo keh njit?

Ttiạ seh tsuh kukwutạdhud

Sun thun nyih ntletutilhchi.

\section{VIII.}

7's.

Christ vuh kooli.

Hampton.

1 Odhudhohtshei tinjihnut Zyeh ket kutchin ungitli Hakthuinjo tsuh ungitli Hakthuinjo vuh koọli tsut. 
2 Vittukoochanchyo Tinji

Tinjih eh njit vuh koọlị

Ttsinettun nunh kug koọi

Chetrsikoonchyo tinjih tsut.

3 Tutthug nunh kug kutchinnut

Rsihkoonchyo ha khuchohzi

Sso zyunothlhi, untohthli

Zyeh chilig nikyo ohthlị.

4 Christ nunh kuggu vuh koọị

Tinjih tetizi keh njit

Tinjih tachya kkwa keh njit

Kọ vih nukwitetilya.

\section{IX.}

Nukwazi chigachil eh njit.

1 Christ vih tsyahnut, nindelh tekit

Nazzi nunh kug zyungai

Zut suh Assi sib tsih chozi

Nichin seh njit gichinchi.

Etshị kwu ttsun kwinjik ndyothtsei

Kwunzi nirzi tthul kwizhit

Nichin tuchun kwundai nasi

Nukwazi koọlị

Chigachil nutrutazi

Chigachil nutrutazi

Chigachil nutrutazi

Nukwazi koọlị.

2 Zyeh zit sih zze kwịrzị kooẹ

Sheggu keh njit kwuteä

Kwugguh yoo sheggu zut tihtya

Rsikotitinyoo nunh kug

Etshịkwu ttsun kwinjikndyothtseị, \&c. 110 
3 Azyuk etshị kwittelya kkwa Vittukoochanchyo tsyahnut Sheggu kwundai kuntetiya Ako sso zyungitelya.

Etshikwu ttsun kwinjik ndyothtsei, \&c.

6.6.4. 6.6.6.4.

\section{National Anthem.}

1 Vittukoochanchyo choh

Vuh tsuh untotili

Nuwhoh ttri ha.

Attun yoo nuwheh Itsei

Kwintluth tsut nichinttai

Tutthug tsut kukwadhud

Thlih ha nirzi.

2 Vittukoochanchyo choh

Kwịrzị koogwatsi chi

Zyunnuwhoh nyoo

Nuwhet tunyinidhun

Nuwhoh tassi keh njit

Tih Tinji Jesus Christ

Nuwhoh ntlantshị.

L.M.

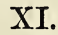

Derby.

1 Ttiạ Kwintluth nyet rsikoonchyo Chunkyọ nirzi suh ntlunyantshi Tsenja tsut kasohtunachya Christ vih kyinjitihtha keh njit.

2 Nersitelhchyo trelhndyoh kirre Chetrsikoonchyo ha sih nyayin Suh troogwandyoh ekoochịli Nih Tinji Jesus Christ kirre.

111 


\section{CHILIG.}

3 Ttiạ kwintluth tinjisihzhit

Tsenja tsut nile kukwutelhthut

Kwịrzi kwikit titihsiya

Ttiạ kwintluth seh tsuh ninyo.

6.8:

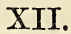

Lennox.

1 Vittukoochanchyo choh Vih chetrsikoonchyo tsut Chilig untotili.

Tutthug nuwhoh ttri ha Vittukoochanchyo choh nirzi Sheggu nuwheh njit chuịnirzị.

2 Attun yoo nuwheh ltsei Sheg nuwhoh kunnatyi Zyungutetizi chi Ako tunnuwhoh nyoo Vittukoochanchyo choh nirzi Sheggu nuwheh njit chuịnirzi

3 Nuwhun yoo zyuntrandyoh Akọ nersintachyo

Tih kkyi nuwhoh ntlantshị

Nuwhoh tassi keh njit

Vittukoochanchyo choh nirzi Sheggu nuwheh njit chuịnirzị

4. Elyet gunitili

Nittso tuntitiya

Vittukoochanchyo choh

Sheg vih kunkwutata

Vittukoochanchyo choh nirzi

Sheggu nuwhoh tsut chuịnirzị. 
C.M.

1 Chootyin tinjih kwịrzi tị Troogwandyo kwatsi kkwa Ko sheggu etunetle choh Kwịrzị ninjiidhut.

2 Elyet koottsite kwutelhtsya Kwịrzị kettinidhun Kwịrzị tsut kwikit tutedhut Jesus Christ kit tehah.

3 Tinjih eltsei kwah zyuntundai Tutthuggu tih tsyahnut Akọ sheg kooh tsuh zyuntenja Sheg kooh kuntunatyah.

4. Thlih ha kwịrzi zyungittelya Kwịrzi tungittiyin Thlyechi Christ ha guttutatsha Azyuk yette zyeh zit.

1 Chunkyo Rsotitinyoo Nuwhoh ttodh kwinịzit Tsenja tsut kunnuwhoh nyạtun Nittsọ kwintluth ịrzị.

2 Kwirzoo tunitiyin Ei ggut nersintachyo Nuwheh nkyo nuwhoh guạnttri Christ tsut nuwhoh nịa. 
3 Nuwhoh tsuh trunyundai

Nih itunetle choh

Kwịrzị kwikit tuntzidhäe

Nih kunkwachi keh njit.

4. Vittukoochanchyo choh

Vih chettigwinidhun

Thlih ha kazyunitundai chi

Ttai ha nuwheh nkyo zit.

5 Nuwhoh kunnuantrhah

Troogwandyoh tutthug ttsut:

Rsikotitinyoo nuwhah ntsi

Yette zyeh zit keh njit.

6 Chunkyọ Rsotitinyoo

Nuwhoh ttri zit kwịnchin

Kwittri kechid ha nuwhah ntsi

Zyuntitirzyah keh njit.

7 Nuwhoh tsuh trunyundai

Nuwhah zyuntitundai

Kwittyi choh, $K$ witinji, nun ha

Chihthloog rzị zyunohthlị.

8.7.

$\mathrm{XV}$.

Ramak.

1 Ttia zyeh kuggu nih kwilnjik

Kwintluth tsut nersitelhchyo

Chetrsikoonchyo ha sih nyạyin

Nersichachyo soh chịnyoo.

2 Troogwihti kwirzoo ttihsiyin

Nih kinjik thletchidhelhnei

Ko Jesus seh njit tettiyin

Nersichachyo soh chịnyoo. 
3 Kwinititaë kug gulttsuk Suh troogwandyoh $\mathrm{k}$ winịkdhei Jesus Christ suh tassi keh njit Ttsettinịdhud, ninidhud.

4. Ttiạ sso nih ttsun telhtsya chi Kwintluth seh tsuh trininji Suh troogwandyoh ekoochịli Nih kki Jesus Christ kirre.

P.M. XVI. Geneva.

1 Vittukoochanchyo nuwheh ltsei Vih nindelh tsut untotili Tih 'Tinji Jesus Christ nuwhoh ntlantshị Troogwandyoh ttsut nuwhoh tassi Treshit nuwhehnjit rsinichyo Jesus nuwheh njit ninidhud.

2 Kwintluth nuwhet tunyinizhin Christ nuwheh njit ninidhud tle Chunkyo nirzị nuwhoh ntlantshị Rsotitinyoo nuwhoh tatsya Attun yoo nuwhoh tsuh nyoo chi Ette thlih ha zyuntitizi.

$3 \mathrm{~K}$ wittyi nuwhoh tsuh trunyundai Kwịrzị kwikit tuntitudhut Christ nuwhoh ttri zit tivichyo Ttai nuwhoh tsun tatsya keh njit Akọ kunnuwhoh tunachya Nih choogoolloo kug todichya. 
8.7.4.

\section{XVII.}

Helmsley.

1 Zyunkwunohyin kwuttọ nehah

Ei nuwheh njit ninidhud

Jesus kko ha kwutto rehah

Kwintluth lyeịnut yah netah

Alleluia, Alleluia

Nuwhoh Assi kunetik.

2 Choog tutthug zyungitunaya

Nindelh ha nugootazi

Ei elyet yinjigichaä

'Tuchun kug gungiyilttsuk

Kwintluth ttrhe ha

Thlih ha zyungitunaya.

3 Einut nersingichachyo yoo

Chih nunh kug kookwundai taị

Jesus Christ ggut tunginizhin

Chooggoo zyungutetizi

Sso zyunohthlị

Jesus Christ nuwhoh tassi.

4 Attun nizhyook nunnoovayin

Nindelh ha kwuttọ nehah

Tih tsyahnut ssozyungị thi th

Sheg zyeh zit guttutatsha

Alleluia,

Christ vuh tsuh untotili.

s.M.

\section{XVIII.}

St. Augustine.

1 Chunkyo Rsotitinyoo

Akọtinyoo anei

Vittukoochanchyo vih chehnyoo

Kwikit tunnuwhah nyoo.

116 
2 Chootyin tte odheltshei Tinyachyo yah koochin Kwuttutthug akotetanja Jesus Christ tsuh anei.

$3 \mathrm{Kwẹe} \mathrm{chihseih} \mathrm{nidhun}$ Kwẹe taheih tsut etchi Akọ choọ kwundai tetinja Jesus yoo anei nyoo.

4. Khuntet kunutihtik Akọ tunnuwhah nyoo Nuwhoh Assi nunnyoodhayin Jesus khuntet anei.

\section{XIX.}

1 Nunh kug huịnjitridichi Tzet chun rsretetridhichil Zyeh zit sheg sso kooli. Rsikoonchyo kwittelya Rsikoonchyo, rsikoonchyo Rsikoonchyo kwittelya Chun rsretetudhituta.

2 Thlih ha sso zyuntitilya Jesus zyuntunnaya tai Zut sso koọli nunh kug.

Rsikoonchyo kwittelya Rsikoonchyo, rsikoonchyo Rsikoonchyo kwittelya Chun rsretetudhituta.

\section{Happy Land.}

1 Yette nunh kug kwịrzi Nizhit, nizhit 
CHILIG.

Nichin Christ vih tsyahnut

Nungichilzi

Kwịrzị tsut chitritli

Jesus vih nindelh tsut

Christ nuwheh Kukwadhud

Sheg sheg nirzị.

2 Sheggu sso zyungịlị

Christ vih tsyahnut

- Vittukoochanchyo tsut

Sheg ungitli

Sso koolị kwittelya

Zyeh zit nuwhoh tekit

Ssokoọlị kwittelya

Ti Assi ha.

1 Vittukoochanchyo choh chuịzi Huị vuh ntluntechitäe Kwintluth nuwhet tunyinizhin Tih Tinji nuwhoh ntlantshi.

2 Christ ti troogwandyoh kwinịkdheị Kwintluth huịjuthudhut Tuchun nikito kug gulttsuk Nuwhoh kwutandai keh njit.

3 Sheggu kwundai hugwittäei Gih kyinjichyenut eh njit Jesus Christ nih kyinjidichi Nuwhoh tutthug nyạssi chi.

4. Chunkyọ nirzị nuwhoh ntlantshi Nuwhoh ttri zit kwichin chi Kwịrzị kwikit tuntitudhut Sso nih tsun tatsya keh njit. 118 


\section{XXII.}

u.M. Nukwazittrin zit. St. Nicholes.

1 Jesus nuwhoh tsuh kukwadhud Chih nunh kug nukwazi tirin zit Kwintluth kuttunyinitudhun Zyeh zit kwiyendo nukwazi.

2 Ei keh njit tunyinjidichi Kookuttunyinitudhun tsut Zut elyet chuntudhitunda Elyet huịjitudhituta.

3 Zut troogwandyoh kwittelya kkwa Tsik etshị ha kwittelya kkwa Jesus nuwhoh tsut zyunninyo Zut zyeh zit kwu tsuh titutah.

\section{XXIII.}

7's. Nukwazi ttrin zit. Blessing.

1. Chih ttrin zit Christ vih ttrin zit Nuwheh njit nimidhud tlẹ Chih ttrin zit nuwhoh Assi Tthunkit kwu ttsun ninechin.

2 Choog ttrin Kukwadhud kweltseị Choog ttrin seh ttsun nilị nyoo Zyeh zit sso koọlị koọlị Akọ nunh kuggu tthui chi.

3 Chih ttrin zit Christ vih tsyahnut Kih nindelh tsuh ungitli Chihthlud nuwheh njit elchị Jesus chun nutedha kkwa. 
4 Kukwadhud nukwutundai

Tutthug kwuttai ttsun nilị

Zut zyeh zit akọ nunh kug

Tih tsyahnut tassi keh njit.

5 Jesus Christ sih njichäë

Akọ sheg sih kunyạtyi

Kwintluth sih tsuh trininji

Nih kukwutelhthut keh njit.

7.6.

XXIV.

Dunkirk.

1 Sheggu Vittukoochanchyo

Nih tsut ettitihthlya

Nih chetrsikoonchyo keh njit

Kwintluth tsut sso ihthli

Nih chettigwinidhun tsut

Zyeh kug Kukwadhud choh

Sso ha ket kwutulhnduk yoo

Akọ nih titelhttsi.

2 Ttiạ rsye gichöei tạ

Nechịei taị ei tthui

Nih tsuh hugututihchya

Sso ha soh tudhantsha

Nih Tinji Jesus Christ kirre

Tetizi soh chịnyoo

Suh nkyo tsut nyakwuth anei

Akọ nirzị sạntsi.

XXV.

6.8's. Baptiznyitinya Rsotitinyoo. Eaton.

1 Nuwhoh chassi kwu tsut Kwitshid Einut tutthug nyooh rzi kwu tsut 120 
Choọ ha kooh baptiznitunja Chetrsikoonchyo ha kooninji Zyeh zit gututatsha keh njit Kwunkyọ zit kooh rsyeninyanji.

2 Nunh kug troogwandyoh ha kwu ttsut Rsyunkotitetinja keh njit

Rsittuttsut kooh kunyantyi chi

Akọ sheggu kooh tsuh trunyundai

Kwịrzị tsut tungitetiya

Akọ sso nih tsun gutatsya.

3 Tsenja tsut kunkoohnyatun chi

Nuvetshid kuhnugutatul

Nihut tunginitudhun chi

Akootinchyo tinjih tutthug

Thlanchi kazyungutetundai

Nih Kukwadhud thlih ha nirzị.

\section{XXVI.}

7.6. Ninjikwudhud rsotitinyoo. Ewing.

1 Zyeh kuggu kwu ttsut thlyeth chyo Nyuh kkọ zyunkwitundai Thlih ha nih tthui ssih nili

Thlyeth chyo kookwundai yoo

Zyeh kug kwu tsut chug choh chun Nyuh tta gitunja yoo,

Nuwhoh troogwandyoh kwu ttsut

Rsyun nuwhoh tạnja ggut.

2 Ttrin ndo thloogootunazya

Ttai kunitiyin chi

Ei nuwhet njit ninidhud

Kwundai chile kirre 
CHILIG.

Kookwundai tsuh kukwạdhud

Nih ttsun zyunitili.

Huịnjidhịtudhut kirre

Zyunkwutitundai chi.

\section{XXVII.}

L.M.

Nukwazi Ttrin zit.

Angels.

1 Seh Kukwadhud thlih ha nirzi Nyooh rzi tsut huị ha chitritli

Nih chettigwinidhun keh njit

Kwittri ha kwịrzị trigịkhyi.

2 Vittukoochanchyo vih tsyahnut

Nazzi ttrin zit rsotitinyoo

Kwintluth tsut ket tunginidhun

Akọ sso ha gih kunnatyi.

3 Nyakwuth kwinilhyin, koochihtthuk

Ako tutthug kasuhndai chi

Chitti nunh kug tinjisihzhit

Thlih ha sheg keh njit sso ihthlị.

4 Azyuk yette sheggu nunh kug Sso ha Christ vih kukwutelhthut Christ chịnttsi sheg kwutunelhya Akọ veh njit sheg sso tihthlya.

\section{XXVIII.}

L.M.

Kwinitrinyanjik. Rockingham.

1 Vittukoochanchyo suh dhantsei

Suh ttrin kwilikwutedha tai

Zyeh kug kwu tsuh tihsya keh njit

Kasohnyatun, sih tsuh ninyo. 


\section{CHILIG.}

2 Suh ttrin nittsọ tulkwuth nilị Nittso nitrichihtlok ei tthui

Akọ nittsọ shọ uttitilhzi Ttią tsenja kasohnyatun.

3 Kwintluth nih tsut gittinihchi Chiyendo tsut nyet tinihthun Nih kinjik akọtelhyin chi Akọ sso nih tsun telhtsya chi.

L.M.

\section{XXIX.}

Wareham.

1 Thlih elyet suh troogwandyoh ggut Jesus tuchun kug ninidhud Chetrsikoonchyo kakwetundai Chettigwinidhun chịnttsi ha.

2 Jesus kwintluth set tinizhin Chitti veh njit titihsiya Suh Assi nih ntlutitilhehi Ei rzi kihthun titihsiya.

L.M. XXX. Derby.

1 Jesus kwu tsuh kukwutadhud Nikyo nunh kug rsinichi ha Veh njit sheg trigutitechya Yeh njit tinjih tsëintelya.

2 Tutthug nithlinetsi kutchin Tih chettigwinidhun chinttsi Sso ha kwintluth tunginidhun Akọ sheg gih tsut ungitli.

1 Nichin tte Jesus Kukwadhud Tinjih tet ssokoọlị koọlị Nuwhun vuh tsuh untotili Akọ veh njit sso zyuntoli. 


Microlomad by -

Proservation

Senvicos

$x^{c} 6516.06$

1 Moves 


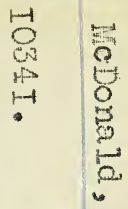

$i$ 


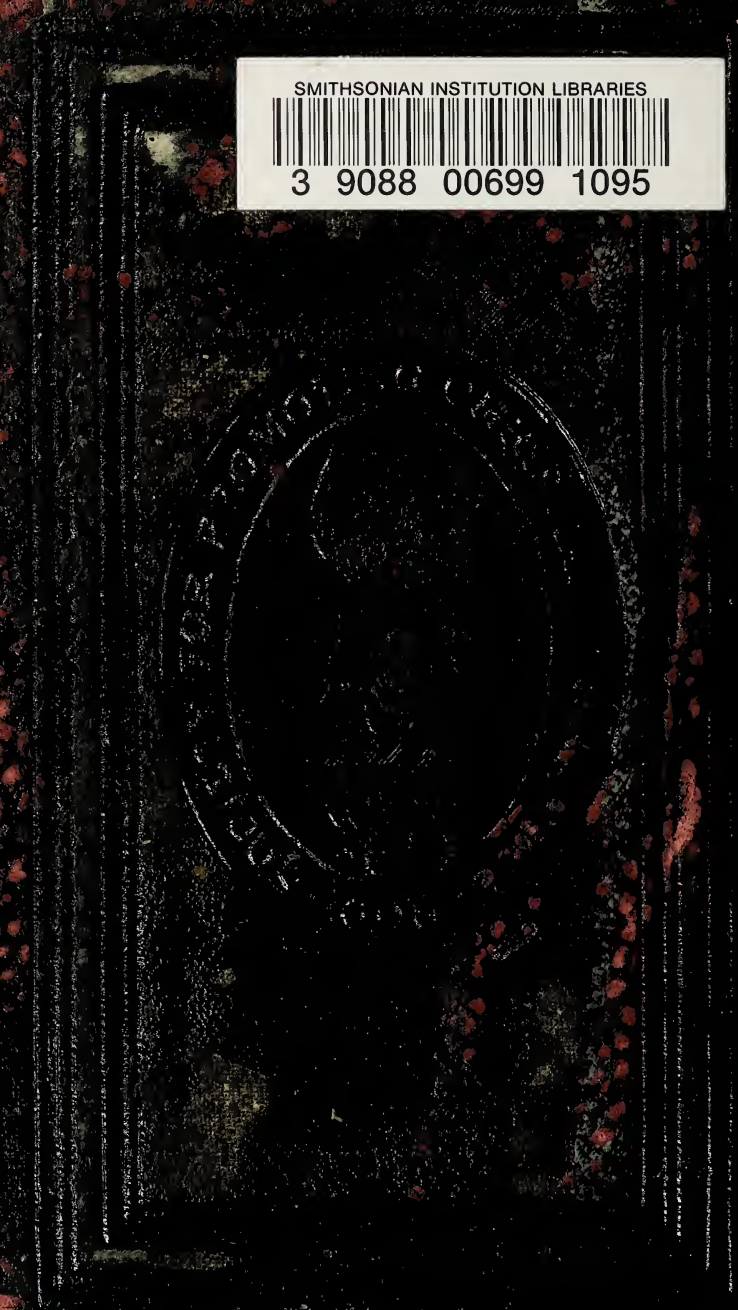

